Article

\title{
A Study of Urban Flood Safety Patterns in Built-up Areas of Bangkok Based on Complex Network Theory
}

\author{
Zhou Yingying ${ }^{1}$,Li Shuaibo ${ }^{2 *}$ \\ 1Studying for a Doctorate at the Royal University of Thailand;1154278836@qq.com \\ 2 Jinshui Bureau of Zhengzhou Natural Resources and Planning Bureau; lishuaiboasas@126.com \\ *Correspondence:Shuaibo Li; lishuaiboasas@126.com; ORDID 0000-0001-8398-1534; Tel..+86 15890198897
}

\begin{abstract}
With the development of the city, a large number of water networks in the built-up areas of Bangkok have been filled and hardened, resulting in poor urban flooding and aggravating flooding, causing loss of life and property of citizens. In this paper, on the basis of combing the current water networks and open space potential flood storage points in the built-up areas of Bangkok, the complex network diagram of the water system in the built-up areas of Bangkok is constructed by combining the theory of complex networks and analyzing the attribute parameters of the network and the characteristic parameters of the open space storage nodes and water system paths, and finding that the water system network in the built-up area of Bangkok has complex network characteristics such as robustness, clustering and hierarchy. By exploring the key storage points and water system connection paths, the researchers initially constructed a flood safety pattern in the built-up area of Bangkok with 145 key nodes and 127 river paths as the backbone, and conceptualized the development study of the flood safety pattern in both horizontal and vertical directions. The urban flood safety pattern based on complex network theory proposed in this paper provides a case reference and methodological ideas to scientifically solve the game conflict between the demand for construction land for urban development and the construction area of urban open space storage points and water storage network under the increasingly severe flooding situation.
\end{abstract}

Keywords: Complex network; flood safety patterns; Water Network; Built-up area of Bangkok 1.Introduction

1.1 Built-up area of Bangkok

Bangkok, the capital of Thailand and the second largest city in Southeast Asia, is located in the Chao Phraya River Delta, with a city area of 1,568.7 square kilometers. The Chao Phraya River flows through Bangkok from the north, dividing the city in two parts, and then flows $20 \mathrm{~km}$ south into the Gulf of Thailand. The terrain of Bangkok is gentle, with an average elevation of 1-1.5 meters, and the river network is dense and long. With the rapid urban development and population growth in Bangkok, the lack of planning and arbitrary filling rivers to build the city has resulted in the reduction of water catchment area and poor water network disconnection. Frequent rainfall causes increased urban flooding, which threatens urban safety. As shown in Fig. 1 and Table 1, the comparison of Landsat5 TM and Landsat8 OLI data for four periods selected from 1990, 2000, 2010, and 2015 (HAN Ruidan, 2017), the 
built-up area of Bangkok increased more than one times in 25 years, which is from $406.12 \mathrm{~km}^{2}$ in 1990 to $819.71 \mathrm{~km}^{2}$ in 2015. The first 10 years (1990-2000) saw an average annual increase of $15.07 \mathrm{~km}^{2}$, and the middle 10 years (2000-2010) saw a slight decrease in the growth rate, with an average annual increase of $13.26 \mathrm{~km}^{2}$. However, starting from 2010, the size of the urban built-up area increased dramatically by 2015 at twice the original rate, reaching an average annual increase of $26.064 \mathrm{~km}^{2}$. Rapid urban construction has encroach on a large number of rivers, paddy fields and woodlands which can drain and store floodwater. Bangkok has a typical tropical monsoon climate, with the rainy season usually starting between May and July and lasting until September or even October, with at least 15 days of rain per month. Bangkok is also near the sea, which makes it susceptible to landfall typhoons that frequently cause flooding in the city. In particular, in July 2011, Bangkok was hit by a 50-year flood that killed at least 297 people, left some 110,000 people in temporary shelters, destroyed large areas of farmland, shut down many factories, and caused heavy economic losses. In order to combat frequent flooding, we need to preserve the original flooding network and storage wetlands in Bangkok, while urban development needs to fill the river and occupy the greenery to increase the land for construction, which forms a kind of game contradiction. It is of great practical significance how to increase the construction land required for urban development in the maximum amount under the premise of ensuring the safety of urban flood drainage in Bangkok.

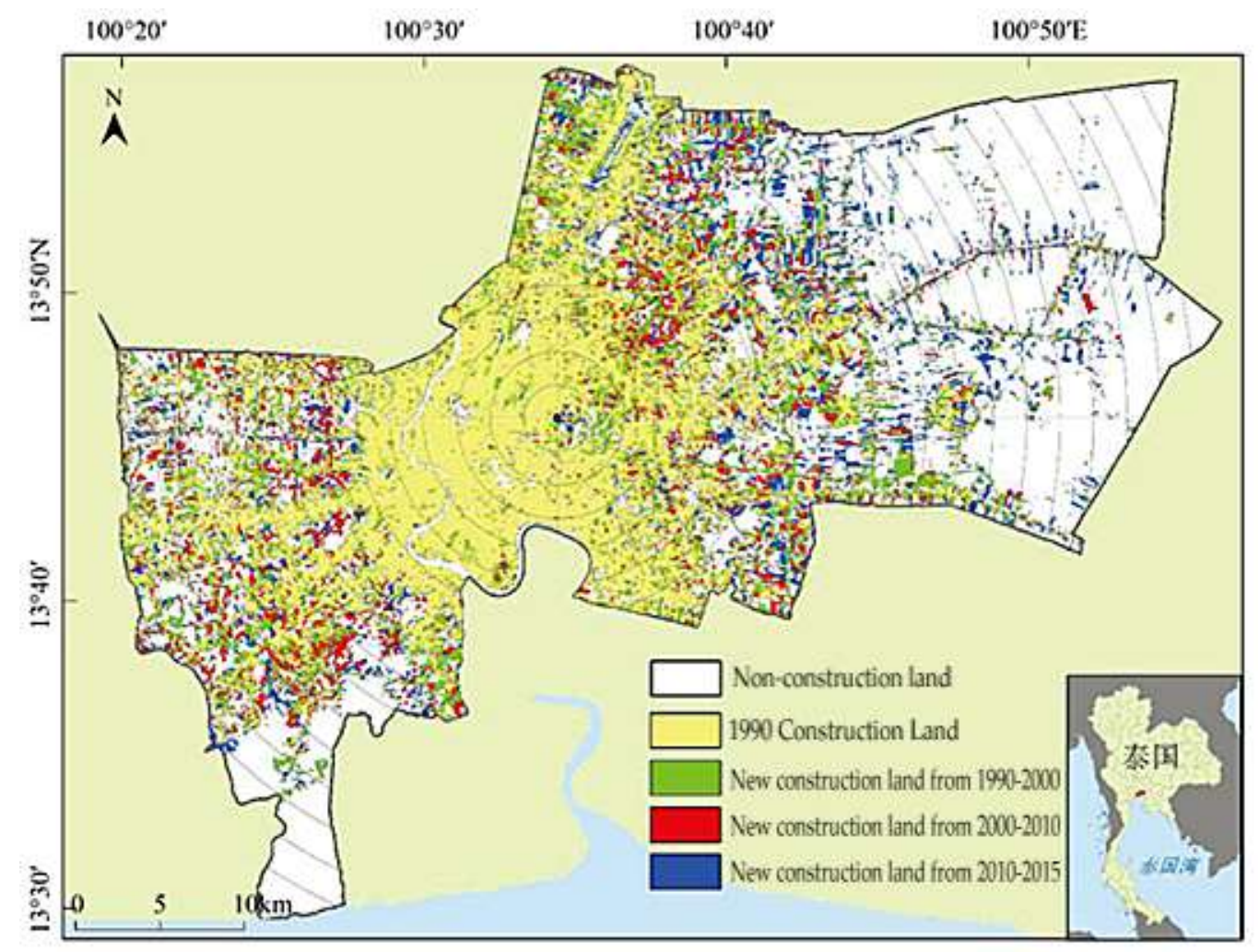

Fig. 1 Map of built-up area from 1990-2015 
Table 1 Change in built-up area of Bangkok, 1990-2015

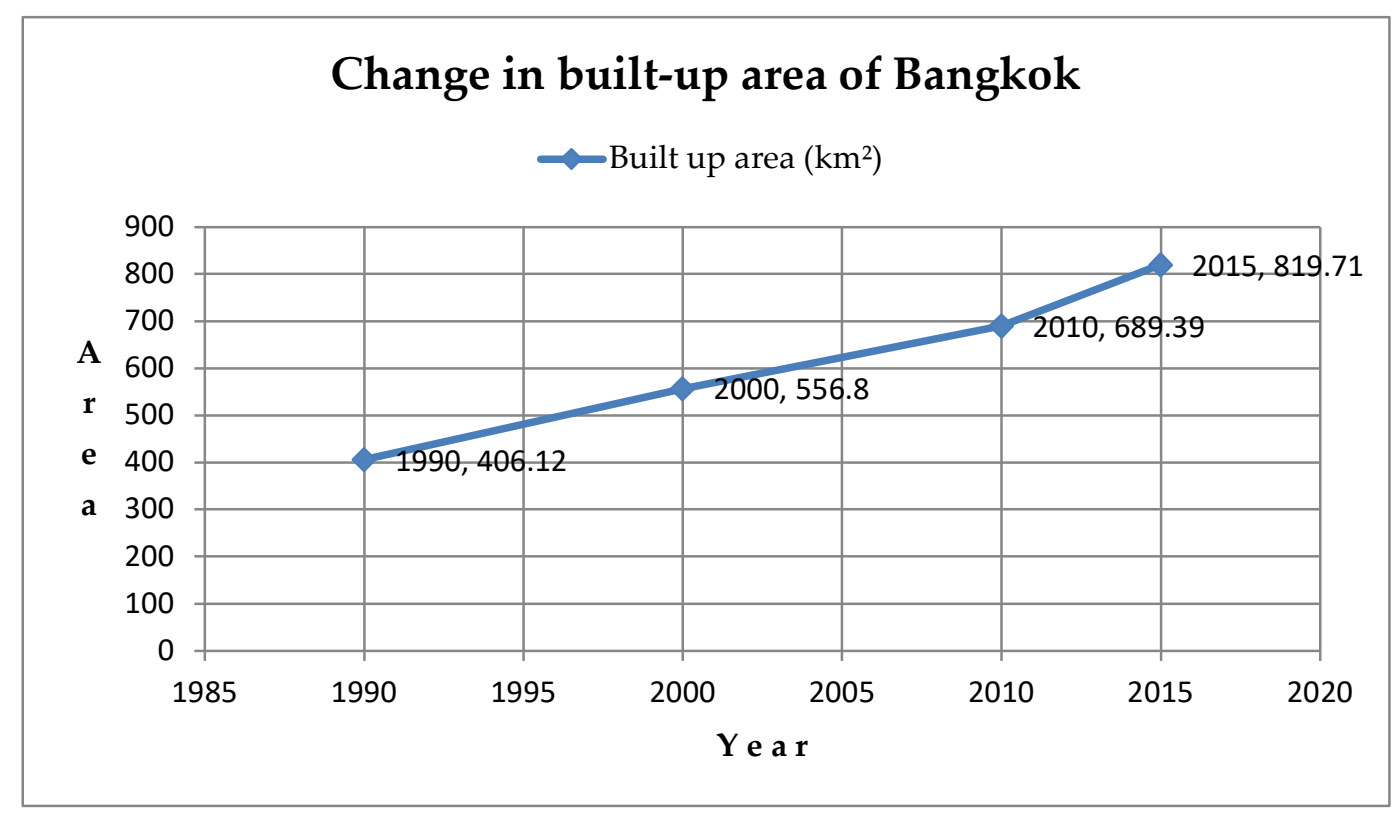

1.2 Urban Flood Safety Patterns

To achieve effective urban flood control, it is necessary to occupy strategic key spatial locations and connections, and the pattern formed by such strategic locations and connections is the urban flood safety pattern. These key spatial locations and connections are open spaces such as green areas, water bodies, squares and wetlands at important nodes in the city and their important contact water systems, which form a coherent and complementary flood storage and regulation system with each other and become a space for natural flood discharge to minimize the impact of flooding on the city. The urban flood safety pattern discussed in this paper is an efficient flood storage system composed of urban open space for flood regulation, stagnation and storage at key spatial locations and river systems with important liaison functions.

\subsection{Complex network}

A large number of complex systems that exist in nature can be described by networks of various shapes and sizes. In the late 1950s, scientists discovered that a large number of real networks are neither regular nor random networks, but networks with statistical characteristics that differ from both, and such networks are called complex networks (Newman, 2003). These networks generally have properties such as small-worldness, clustering, robustness, and power distribution of degree, and small-world network models, scale-free network models, and self-similar network models have emerged. Among them, key node discovery and community cluster discovery in the study of complex networks are important for understanding and studying network structure and function, improving network resistance and enhancing robustness.

\subsection{Summary}

A system is robust if it can maintain its basic functions despite internal and external errors. In networks, robustness is the ability of a system to perform its basic functions even 
after losing some nodes and links. The potential flood storage points such as existing water systems and open spaces in the built-up areas of Bangkok are modeled by combing them using the theory related to complex networks. By investigating the characteristic properties of the network model and the node parameters, the operationalization and feasibility of the network key nodes and community clusters are explored in an attempt to identify the critical areas, spatial locations and links in the flooding pattern of Bangkok. By combining the "robustness" of the network, an efficient flood storage system consisting of open spaces for flood regulation, detention and storage at key spatial locations and river systems with important linkage functions is constructed - a flood safety pattern for the built-up area of Bangkok.

\section{Materials and Methods}

\subsection{Study Area}

The scope of this study is bounded by the artificial surface connected in the CGLC30-2020 Bangkok surface coverage map (Fig. 2), covering the core area and the peripheral area of Bangkok (Fig. 3), with a total area of about $543.5 \mathrm{~km}^{2}$. The core area, with Bangkok City Hall as the center, has a radius of $10 \mathrm{~km}$ and basically covers the core built-up area of Bangkok with an area of about $320 \mathrm{~km}^{2}$. The outer zone extends $10 \mathrm{~km}$ to cover Bangkok's Langman International Airport in the northeast and the former capital of the Thonburi Dynasty on the west bank of the Chao Phraya River in the southwest, as well as Late Sukhumvit in the southeast, forming the outer zone with an area of about $220 \mathrm{~km}^{2}$ (Fig. 2, Fig. 3). The northeastern and southwestern built-up areas of the periphery have been severely flooded in recent years, especially in the July 2011 floods, which were the most severe, as shown in Fig. 4, with darker colors indicating more severe damage (exclusive secrets, bangkok's next 7 potential investment golden places!, 2019). The built-up areas along the Chao Phraya River in the northwest and south of Bangkok are not part of the Bangkok city limits in terms of geographic jurisdiction, and large areas of agricultural land and water bodies exist in the eastern suburbs, and the built-up areas are severely fragmented; these two areas are not included in the scope of this study.

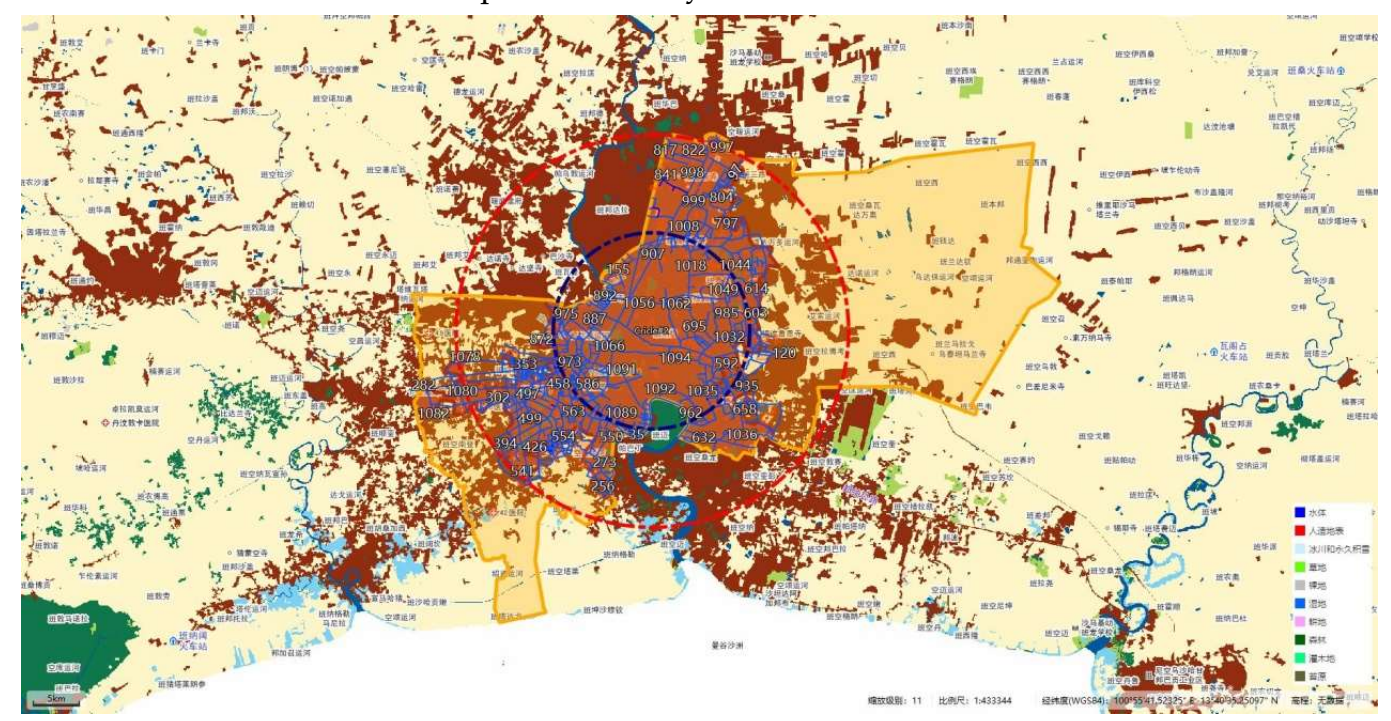

Fig. 2 Study area of the built-up area of Bangkok 


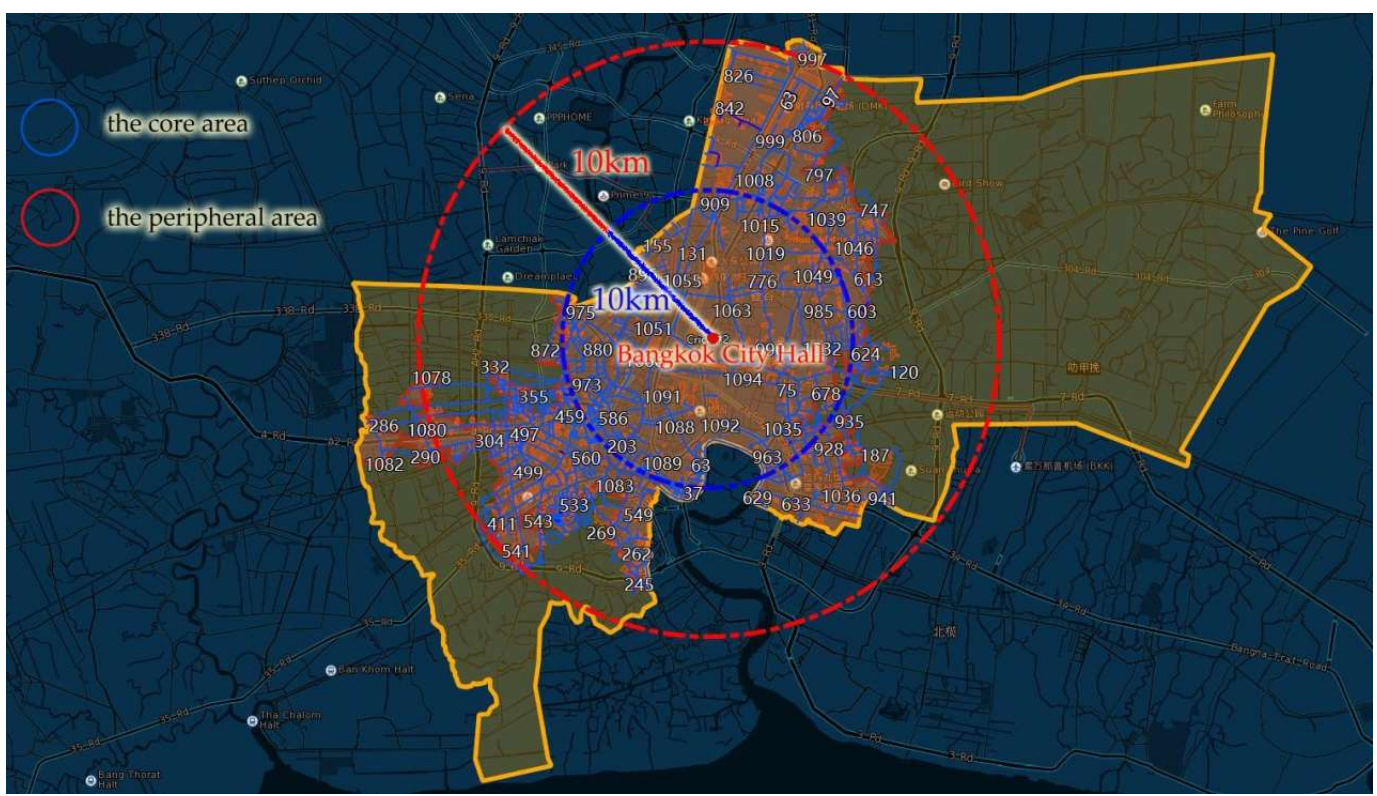

Fig. 3 Distribution of water network and storage points in the built-up area of Bangkok

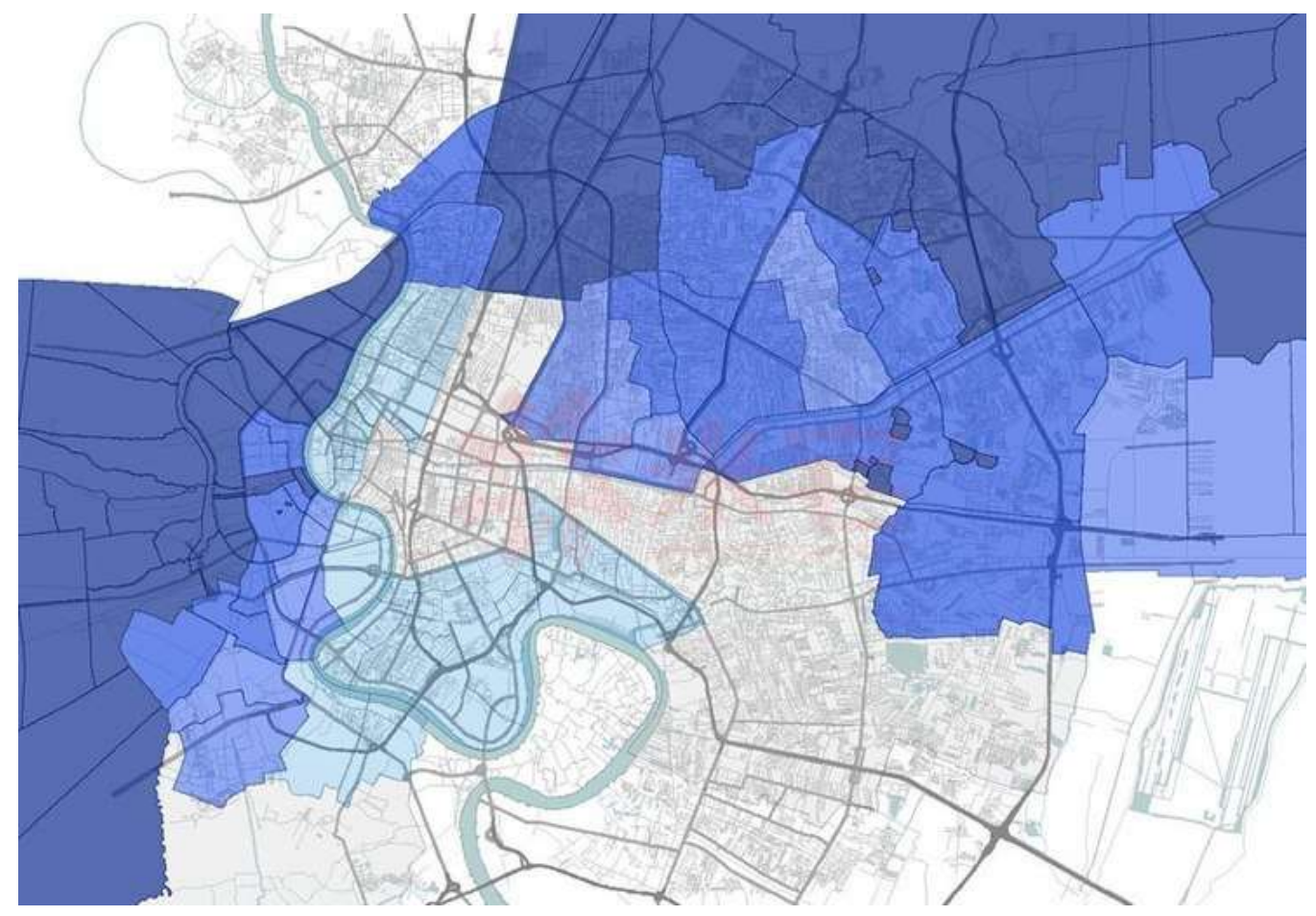

Fig. 4 Bangkok disaster map in 2011

Statistics using Google earth Pro and MegoMap software show that there are 586 sections of water systems within the study area, with a total length of $960.7 \mathrm{~km}$ (shown in blue in Fig. 3), with an average of $1.77 \mathrm{~km}$ of water network per square kilometer; 1,087 open spaces of various types of green areas, open spaces, squares, large parking lots, water bodies, woodlands, stadiums, and farmland in the fringe area, with a land area is 77.3 square kilometers (shown in orange in Fig. 3), with an average open space area of $0.14 \mathrm{~km}^{2}$ per 
kilometer and an area share of $14 \%$. Taking the maximum 24-hour rainfall of $300 \mathrm{~mm}$ in mid-September 2020 in Bangkok as an example, the total rainfall per square kilometer of built-up area is 79.2 million gallons. The built-up area has $0.14 \mathrm{~km}^{2}$ of water storage site and $1.77 \mathrm{~km}$ of watercourse per square kilometer. The water storage site can store 73.92 million gallons of water based on an average excavation depth of 2 meters, and the waterway can store 3.51 million gallons of water based on an average width of 5 meters and a water depth of 1.5 meters, for a total of 77.43 million gallons of water per square kilometer. The existing water network and storage points can theoretically accommodate the maximum rainfall in Bangkok during the rainy season and can solve urban stormwater flooding.

\subsection{Data Sources}

Through Google earth Pro's 2019 high-definition satellite film and field verification, the potential 1087 Bangkok urban flood storage points and 586 sections of the current water system were depicted and counted. The KML files were generated using Google earth Pro software and imported into MegoMap and AutoCAD software for vectorization of the storage points and water system paths. The area of the storage node and the length of the water system path were counted, and the CSV file was output, which was imported into Gephi software for processing. Using the area of the storage point as the node weight and 100 times the reciprocal of the water system length as the edge weight, the node, edge and association parameters are counted and visualized.

\subsection{Methodology}

The green areas, vacand land, squares, large parking lots, water bodies, woodlands, stadiums, and farmlands in the fringe areas within the study area are defined as potential urban flood storage points. The area of the storage points is the weight of the network nodes, the larger the area, the larger the amount of water to be stored during flooding, and the greater the weight. The current urban water system that connects each storage point is defined as the network path edge. Considering the urban flooding, it is easy to produce rainwater diffusion, and the flow direction is uncertain, so it is defined as an undirected edge, and the weight of the edge is defined as 100 times of the inverse of the length of the water system (to facilitate numerical calculation). The longer the water system between two storage points, the weaker its flooding capacity and the smaller the weight. Using these storage points and the current water system, a water system network map of the built-up area of Bangkok was constructed.

First, the Gephi software was used to sort out and analyze the important parameter attributes of each node and edge in the water system network in the built-up area of Bangkok, including degree, weight degree, closness centrality, harmonic closness centrality, betweenness centrality, eigenvector centrality, pageranks, clustering coefficient, modularity_class, etc., to investigate the critical nodes and important paths in the flood safety pattern.

Second, through statistical analysis of global characteristic attributes such as network average degree, average weighted degree, network diameter, graph density, modularity, average clustering coefficient, eigenvector centrality, and average path length, we study the 
network structure and functional characteristics, explore the water network model, and lay a quantitative foundation for building a flood safety pattern.

Third, using the results of the above quantitative analysis, the key spatial locations and important water system paths in the water network of the built-up area of Bangkok are identified, and an urban flood safety pattern with key storage points and important water system paths as the backbone is constructed. Combining with the urban development needs and water network characteristics of Bangkok, the flood safety pattern will be adjusted and optimized in time to finally form an open, operable and defensible dynamic hierarchical flood safety pattern to achieve a balance between urban development and flood control.

\section{Results and ana Analysis}

By digitizing the network of potential storage points and water system connection paths in the built-up area of Bangkok, a water system storage network map (Fig. 5) and complex network visualization map (Fig. 6,Fig. 7) with 580 nodes and 1063 paths in the built-up area of Bangkok were constructed. 580 storage nodes have a total area of $72.6 \mathrm{~km}^{2}$ and 1063 water paths have a total length of $905.4 \mathrm{~km}$, and the average width of the water system is $5 \mathrm{~m}$, with a total area of $4.5 \mathrm{~km}^{2}$, accounting for $14.2 \%$ of the built-up area of Bangkok $\left(543.5 \mathrm{~km}^{2}\right)$. On average, there are 1.07 storage points per square kilometer, with an area of 13.36 hectares. The storage points are all calculated at a depth of 2 meters, which can store 70.22 million gallons of water; the waterways are all calculated at a depth of 1.5 meters, which can store 3.31 million gallons of water per square kilometer of the built-up area's water system path, for a total of 73.53 million gallons of water. The theoretical equivalent of $278.5 \mathrm{~mm}$ of very heavy rainfall can be stored.

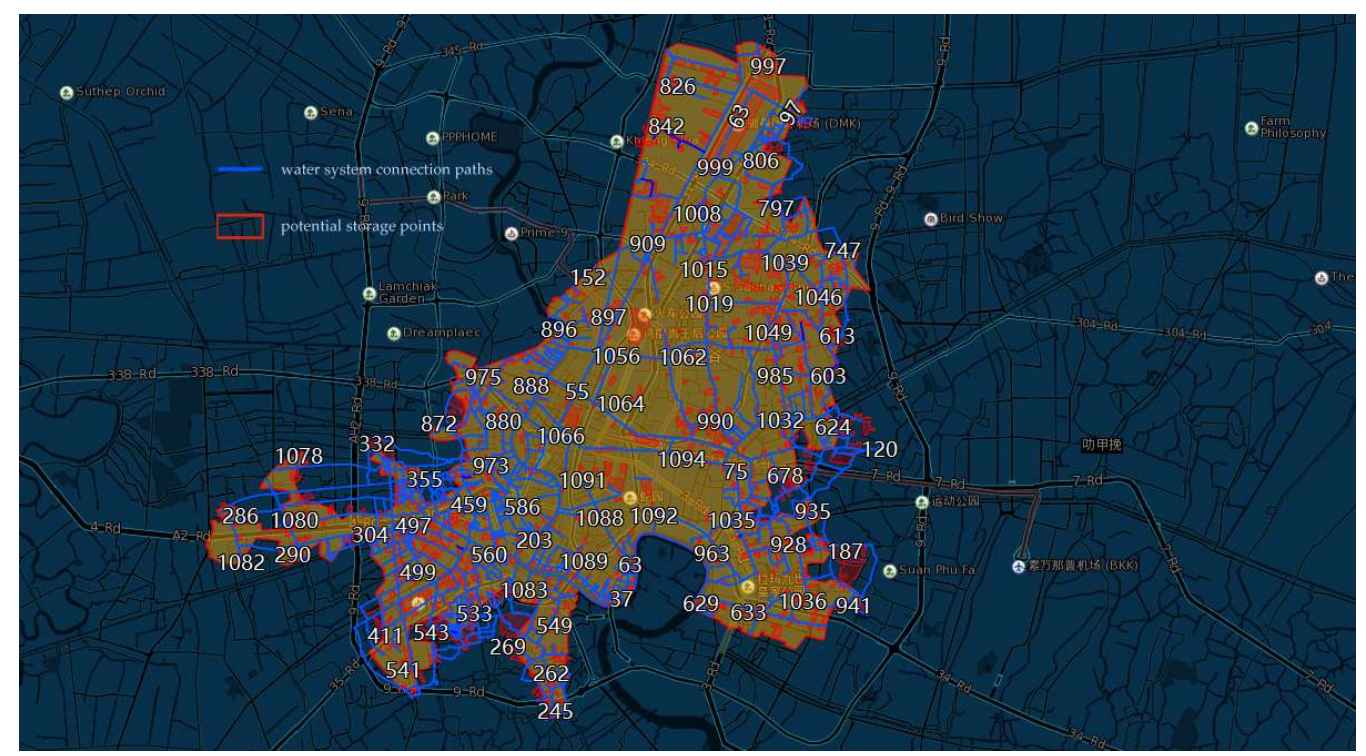

Fig. 5 Map of the water system storage network in the built-up area of Bangkok 


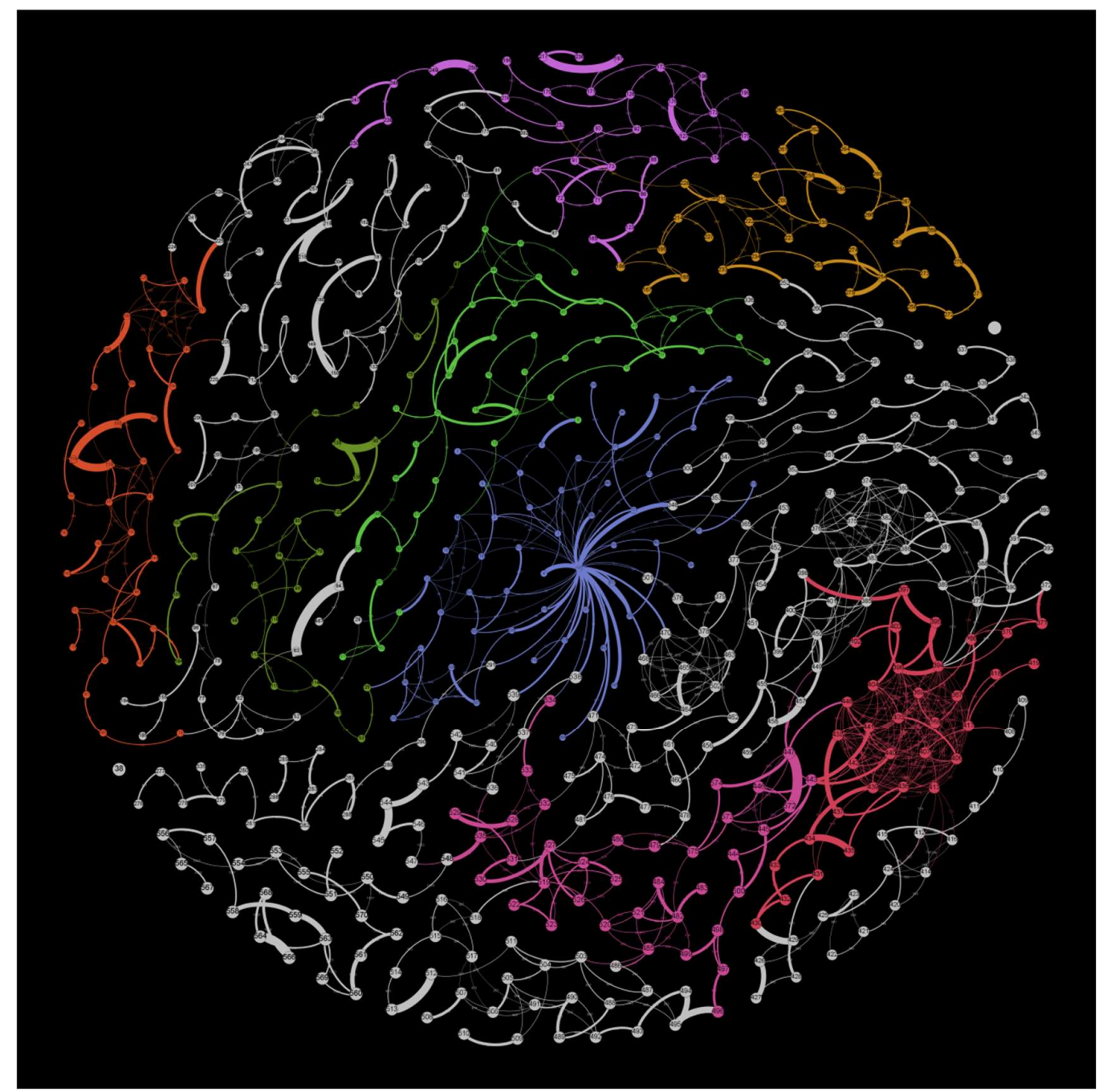

Fig. 6 Visualization of the complex network of storage points-water systems in the built-up area of Bangkok(Fruchterman Reingold Layout) 


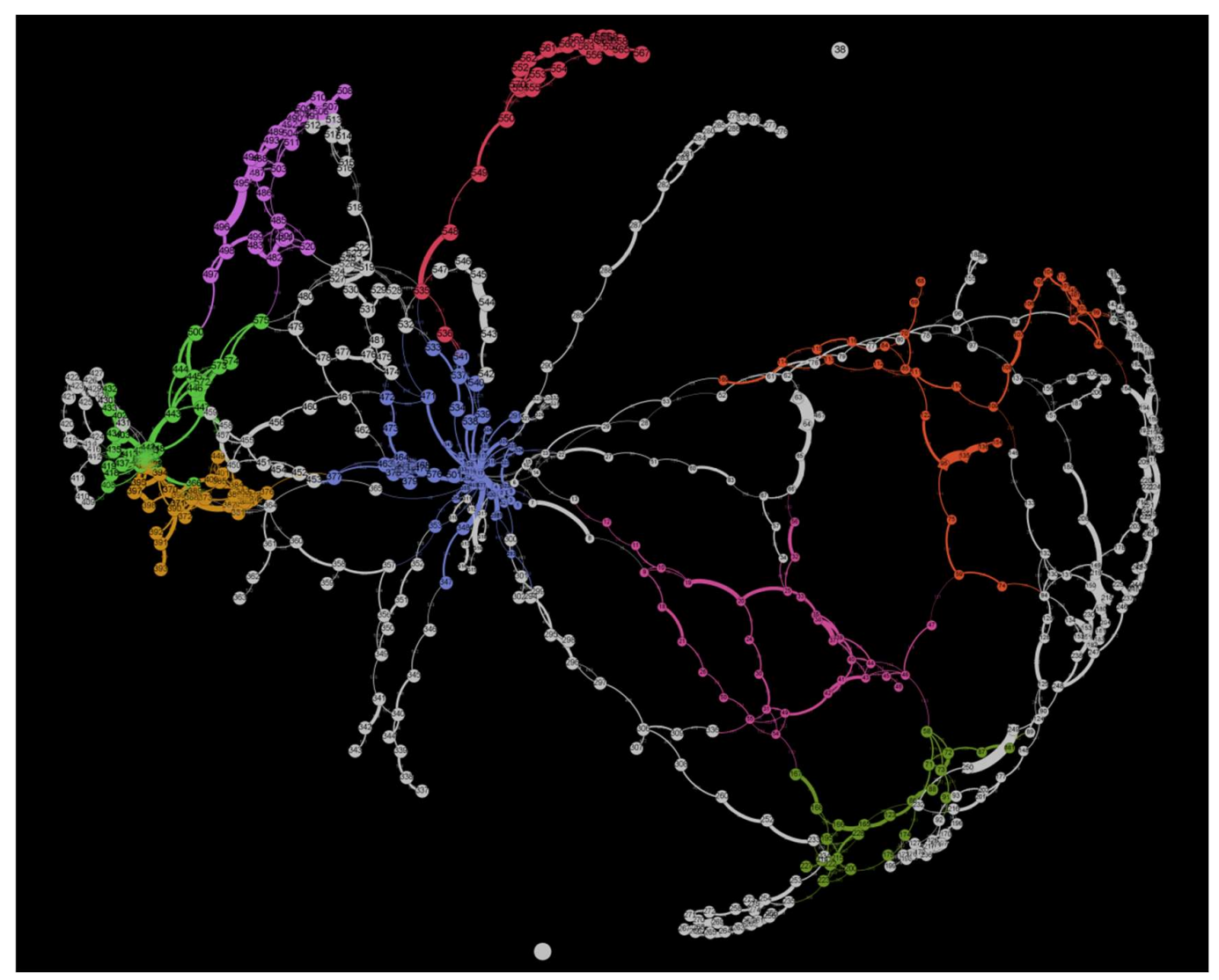

Fig. 7 Visualization of the complex network of storage points-water systems in the built-up area of Bangkok(OpenOrd Layout)

The total length of 1063 path sides is $1589.5 \mathrm{~km}$, excluding the overlapping part of the water system and no connection to the network water system, the total length of the water network path is $905.4 \mathrm{~km}$. The average length of the water system paths between the storage points is $0.85 \mathrm{~km}$, which matches the scale of modern city $0.8 \mathrm{~km}$ neighborhoods and the scale of urban arterial traffic network $(0.8 \mathrm{~km}-1 \mathrm{~km})$, and has a good basis for coupling modern urban pattern, forming a water-land dual transportation system and unique urban structure and appearance.

3.1 Analysis of the main characteristic parameters of the water system storage network

(1) Degree Centrality and Weighted Degree

Degree Centrality is the most direct measure of node centrality in network analysis. The greater the degree of a node means the higher the degree centrality of the node, the more important the node is in the network. As shown in Table 2 and Table 3, the degree values of the built-up area storage points in Bangkok are exponentially distributed, which has the characteristics of scale-free network model, with a few core nodes having very high degree values and other nodes having exponentially lower degree values. In the scale-free network model, the nodes with small degree values have less influence on the structure and function of the network, and their removal does not cause great damage to the whole network, so the scale-free network is robust. There are 19 nodes with degree values greater than or equal to 17 , 
accounting for $3.26 \%$ of the total number of nodes in this network. The three nodes with the highest degree values are node 17 (degree 45), node 394 (degree 23) and node 413 (degree 21). Node 17 is the Chao Phraya River, which runs through the main urban area of Bangkok, where the water systems on both the east and west banks converge into the river, and is the absolute core node in the built-up area of Bangkok and the main channel for flood discharge. 394 is located in the northwest area of the river bank, where the water network is extensive and the storage points are interconnected, and the degree values of several nodes are greater than 17 , forming a cluster of high value nodes. As shown in Fig. 8, there are 37 nodes with degree values above 8 , and this region accounts for 35 of them. The remaining two nodes, 519 and 100, are the core points of the southwestern region and the northeastern region, respectively, and are important key storage points for these two regions.

In the weighted degree statistics in Table 4 and Fig. 9, node 17 (Chao Phraya River) has a larger catchment area and its weighted degree is much higher than the other nodes. The cluster of height-valued nodes in the west is more concentrated, the interconnection path is shorter, the weight of the edge is larger, and its weighted degree is relatively high, forming an important key space in the western region. The southwestern and eastern part of the built-up area in Fig. 9, although the degree centrality is not high, the weighted degree is higher because of the shorter length of the interconnection path of the water system in the region and the greater weight of the edges, which becomes an important node in the region.

Table 2 Nodal degree statistics table

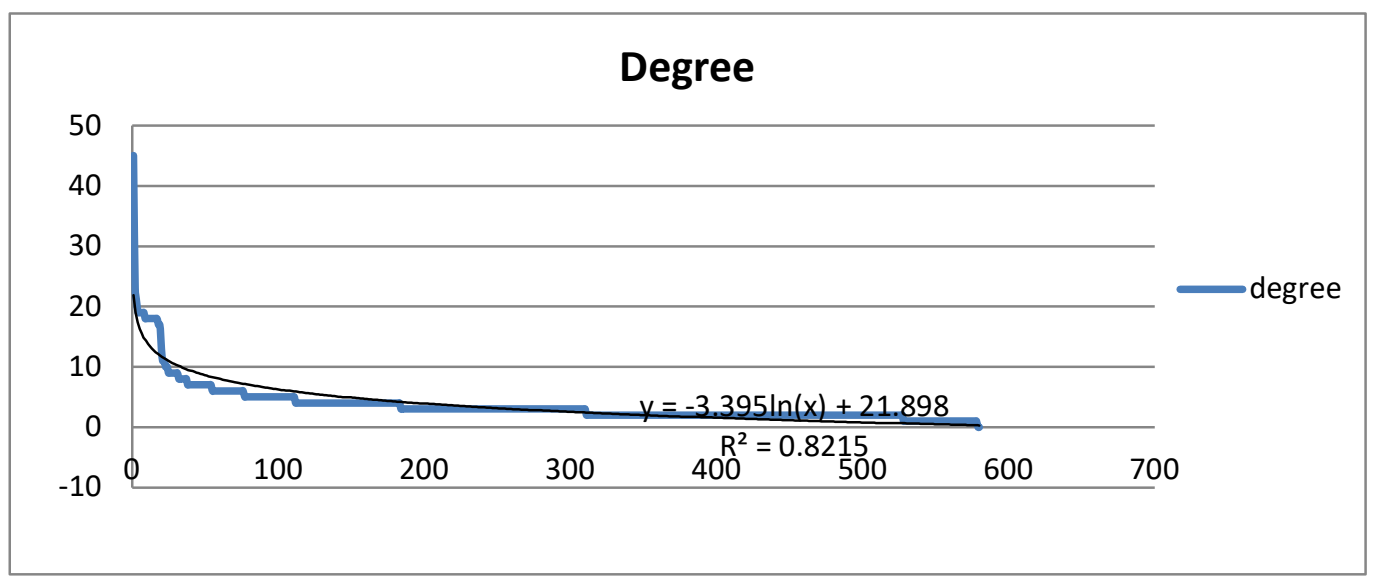


Table 3 Statistical table of nodal degree index
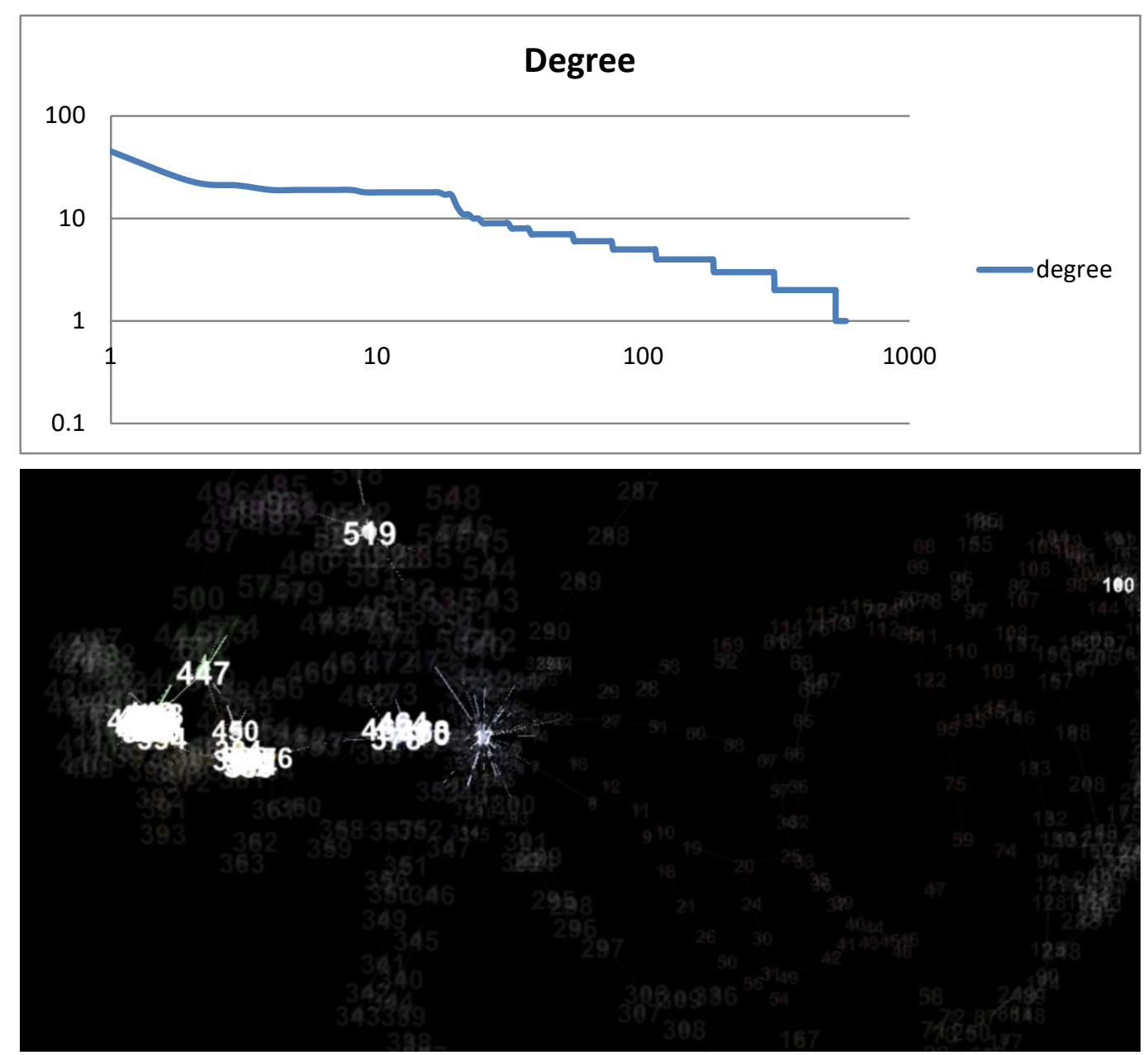

Fig. 8 Distribution of higher degree network nodes

Table 4 Nodal weighted degree distribution table

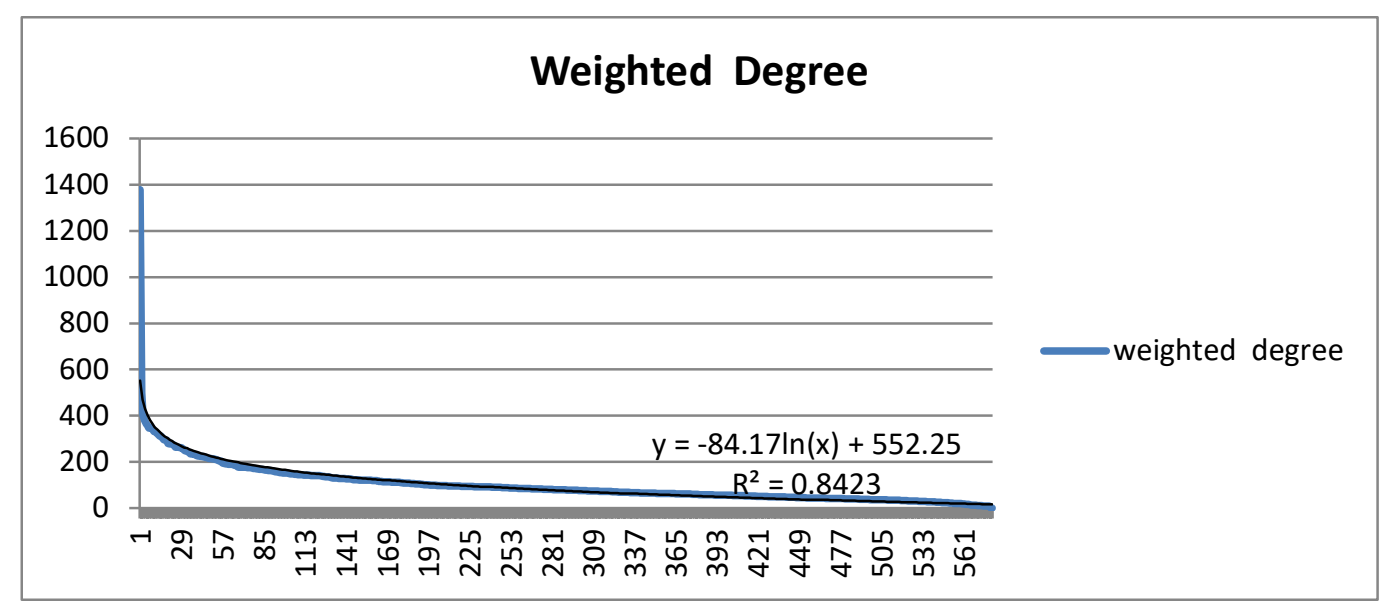




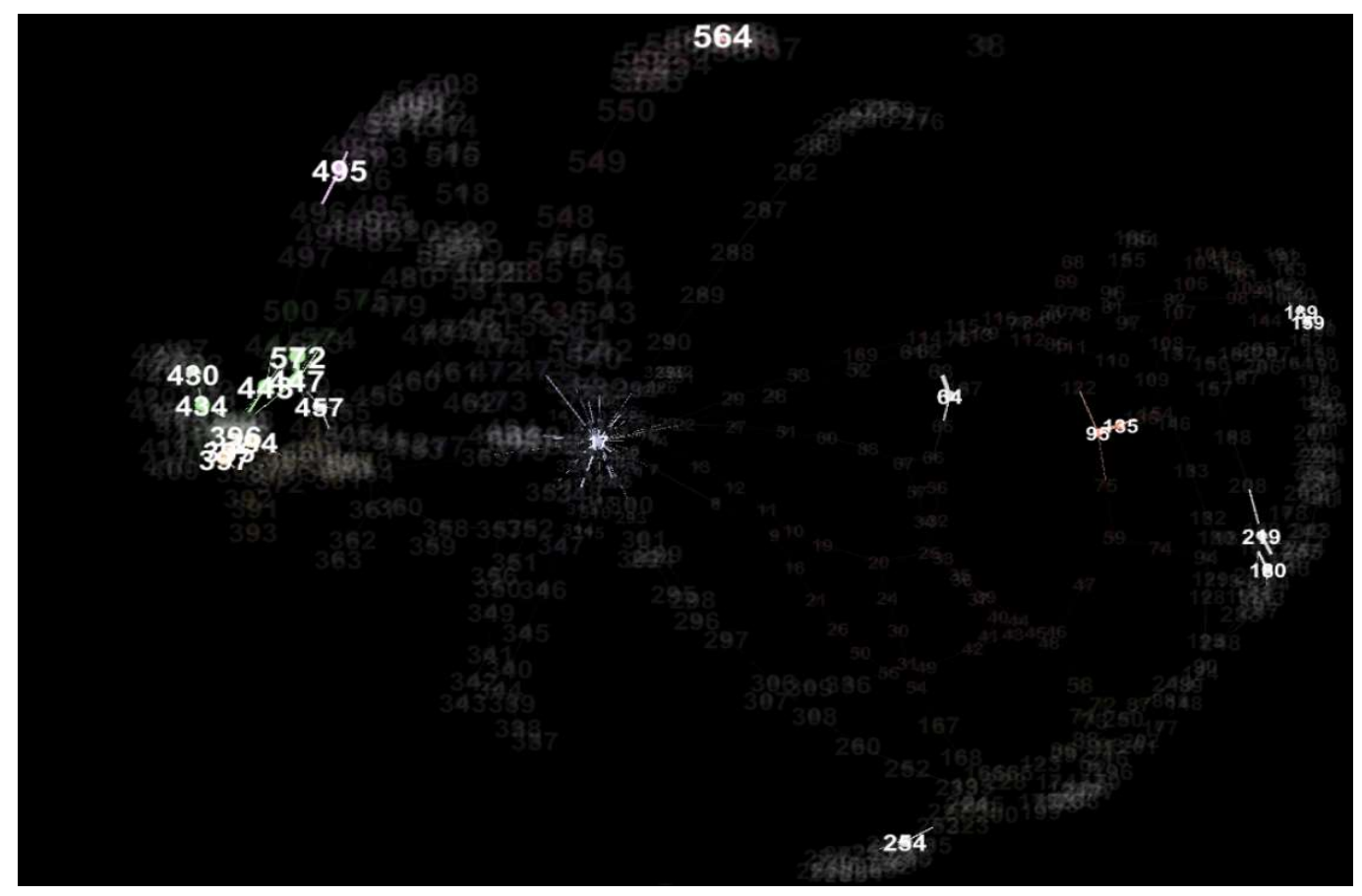

Fig. 9 Distribution of network nodes with higher average weighted degree

(2) Closeness Centrality and Harmonic Closness Centrality

In the field of topology and related mathematics, closeness centrality is a fundamental concept in topological spaces. The closeness centrality of a point is the average of its distance to all other nodes. The higher the closeness centrality of a point, the closer the point is to all other points in the network in general, enabling faster and more efficient material exchange and energy transfer with neighboring nodes. For example, to select a storage point as the core node in a regional water network, so that it can be connected to the surrounding nodes for rapid water storage when flooding occurs, it is required to have the closest spatial distance to other storage points in general, then one way is to find the storage point with the highest closeness centrality. Harmonic closeness centrality is a variant of closeness centrality, which is mainly used to deal with disconnected networks. The overall trend of the closeness centrality and the harmonic closeness centrality values of the nodes in Table 5 are the same. The closeness centrality of node 17 in Table 5,6 and 7 is much higher than other nodes, and the closeness centrality of the first 40 nodes decreases gently and linearly starting from node 22 , which is ranked $2 \mathrm{nd}$, and the closeness centrality of the later nodes decreases faster, forming a cluster of nodes with node 17 as the core and 40 nodes as the sub-center with high closeness centrality. In Fig. 10 and Fig. 11, the nodes with high closeness centrality and harmonic closeness centrality all have node 17 as the core and nodes 22, 23, 365, 471, 300, 14, 16, 7, 15 and 534 as the secondary cores, in contrast to the reality that most of the water networks in the east and west regions are discharged into the Chao Phraya River through the secondary core storage points on the banks of the Chao Phraya River. In Table 5 and Fig. 11, the harmonic closeness centrality of nodes 394 and 413 is also high, and in reality these two nodes 
are located in the northwest region, which are connected to several surrounding nodes in close proximity and are the core storage points in the region.

Table 5 Nodal closeness centrality and harmonic closeness centrality value table

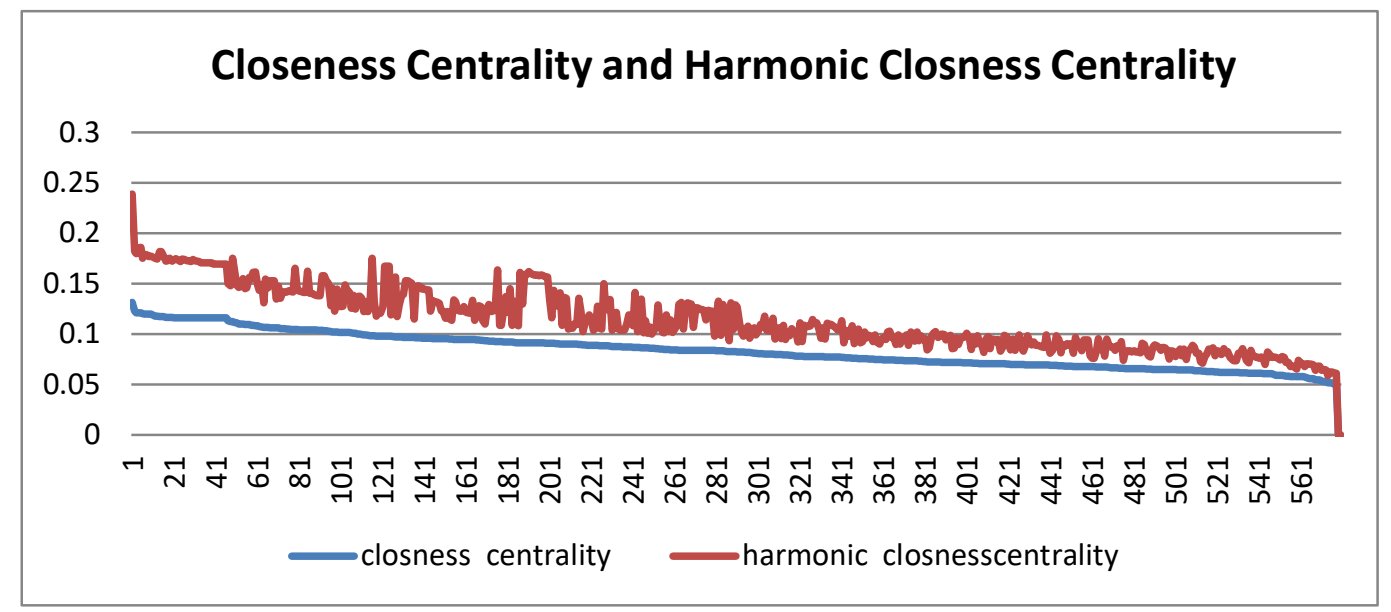

Table 6 Nodal closeness centrality value table

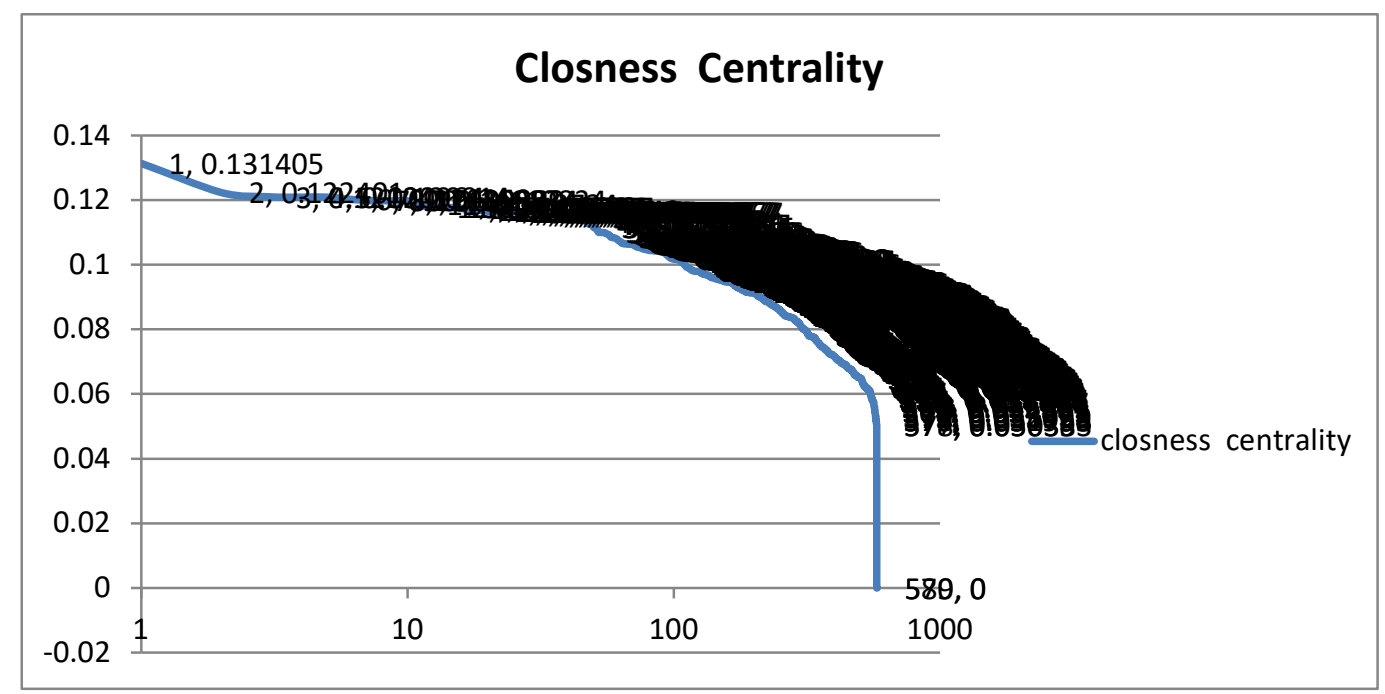


Table 7 Nodal harmonic closeness centrality value table
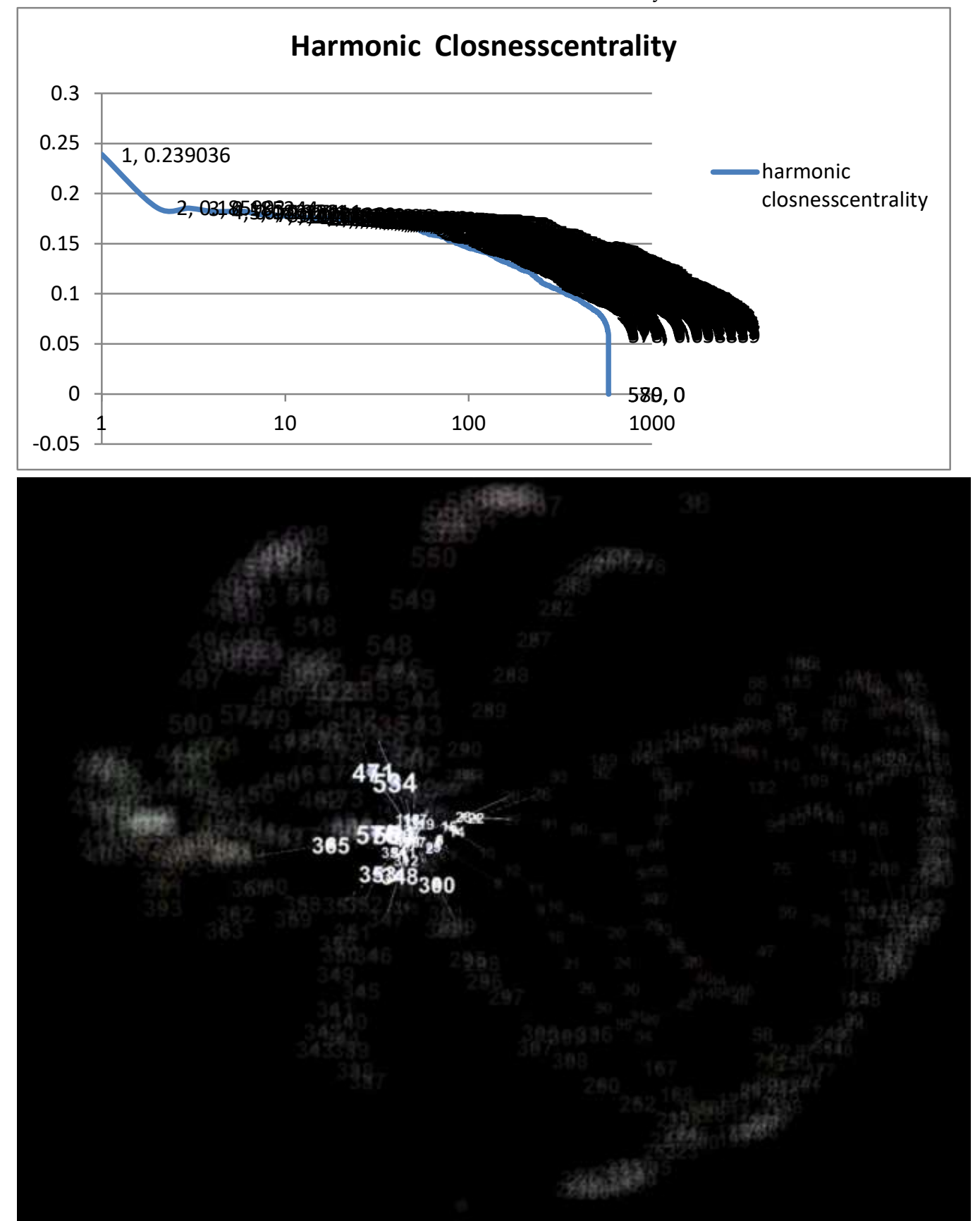

Fig. 10 Distribution of nodes with high values of closeness centrality 


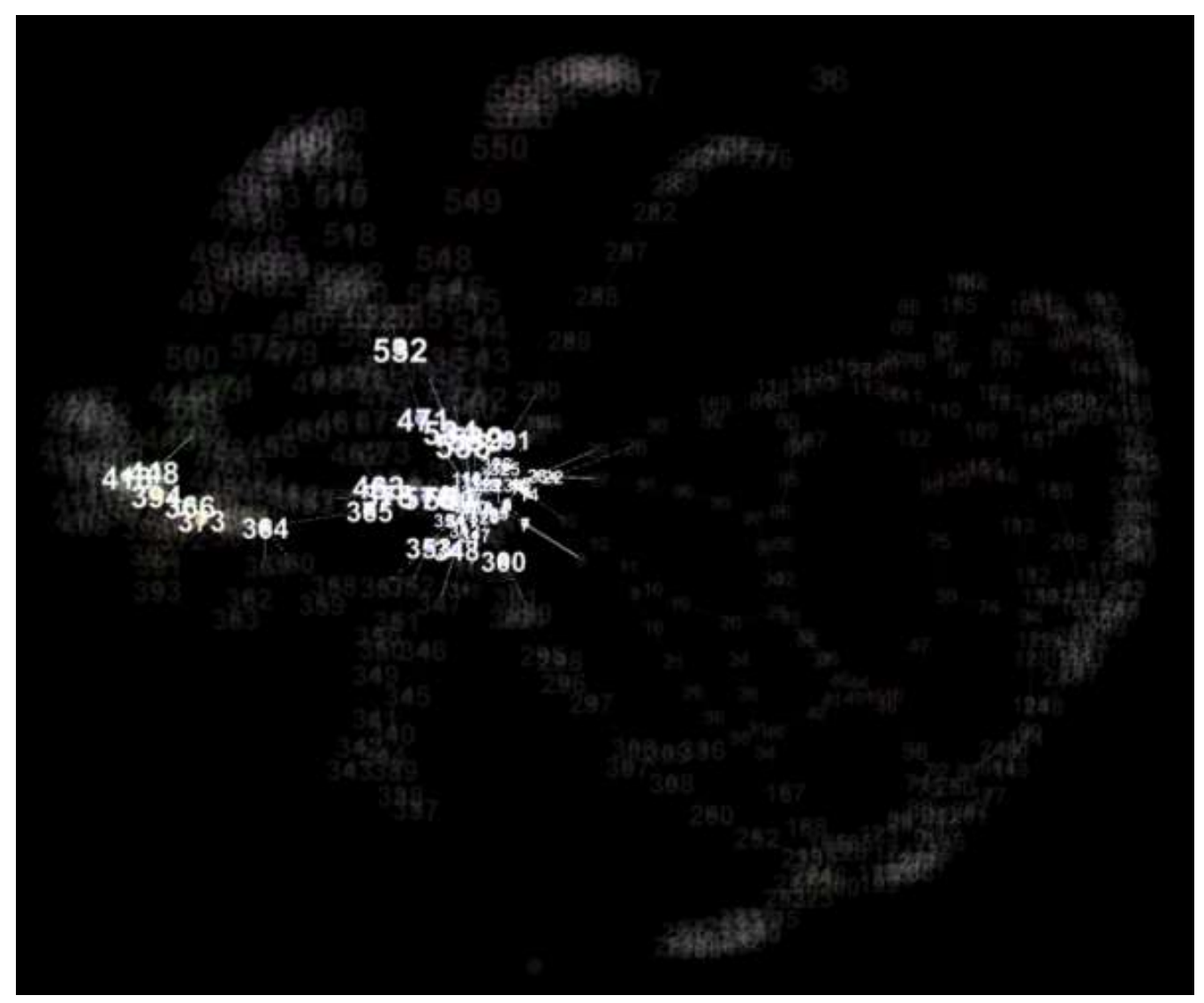

Fig. 11 Distribution of nodes with high values of harmonic closeness centrality

(3) Betweenness Centrality

Betweenness centrality is a metric that portrays the importance of a node in terms of the number of shortest paths through a node, and is the number of times a node acts as a bridge for the shortest path between two other nodes (Newman B. W., 2006). Betweenness centrality is a test of the degree to which a node influences the flow of information in a network. The more times a node acts as an "intermediary", the greater its Betweenness centrality, indicating that many, even all, of the shortest paths between other points must pass through it, and the more important its influence on the flow of information in the network. If this point disappears, then communication between other points will become difficult or may even be disconnected. Table 8 show that node 17 has extremely high values of betweenness centrality, and six nodes, including 364, 365, 22, 471, 28, and 52, also have values over 30,000, corresponding to the status quo in which these nodes are the transit centers of the western and northeastern water networks, respectively. As shown in Fig. 12, the final formation of the node 17 as the core, with 28 high betweenness centrality as the backbone, radiating eastward, northeastward, southeastward, westward and southwestward network of five water flow "intermediary" belts. 
Table 8 Nodal betweenness centrality value table
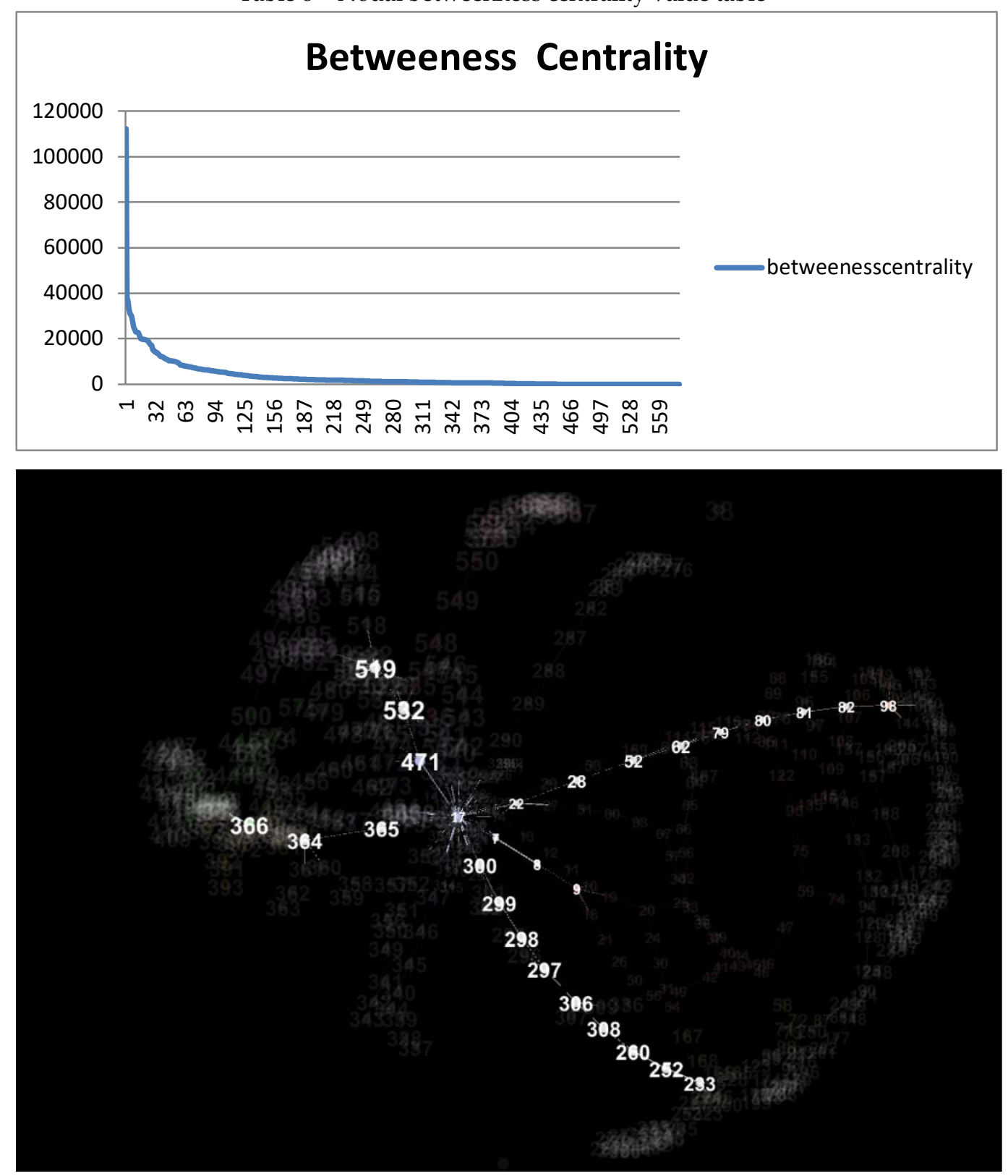

Fig. 12 Five "intermediary" belts of network water flow with high median centrality

(4) Eigenvector Centrality

Eigenvector centrality measures the importance of a node by considering the importance of neighboring nodes, and is an algorithm for measuring the transfer of influence or connectivity between nodes. The main principle is that connections from important nodes (measured by Degree Centrality) are more valuable than connections from unimportant nodes, and that connections to nodes with high scores yield a greater contribution than connections to nodes with low scores. For example, a node with 300 very popular friends has a higher eigenvector centrality than someone with 300 relatively unpopular friends. All nodes 
start equally, but as the computation proceeds, nodes with more edges start to become more and more important. Their importance propagates to the connected nodes, and after several iterations of the calculation, these values stabilize, resulting in the final value of eigenvector centrality (S.Boccaletti, 2006). As shown in Table 9, the eigenvector centrality of the first 18 nodes is greater than 0.9 , and the 19 th node eigenvector centrality falls precipitously to 0.18 . Fig. 13 shows the network visualization of the 18 nodes with high values of eigenvector centrality, which are located in the western part of the Chao Phraya River. The water network in this region is uniformly dense, and each storage point is spatially staggered through each other, and the spatial water network tends to be a regular network model with a good basis for flood storage.Fig. 14 shows the viewable view of eigenvector centrality value greater than 0.1 , and the number of nodes increases to 24 , which is basically also located in the western region. The only node 17 (Chao Phraya River with a value of 0.15 ) is added, which also has a relatively high eigenvector centrality as the core point of the entire network catchment.

Table 9 Nodal eigenvector centrality value table

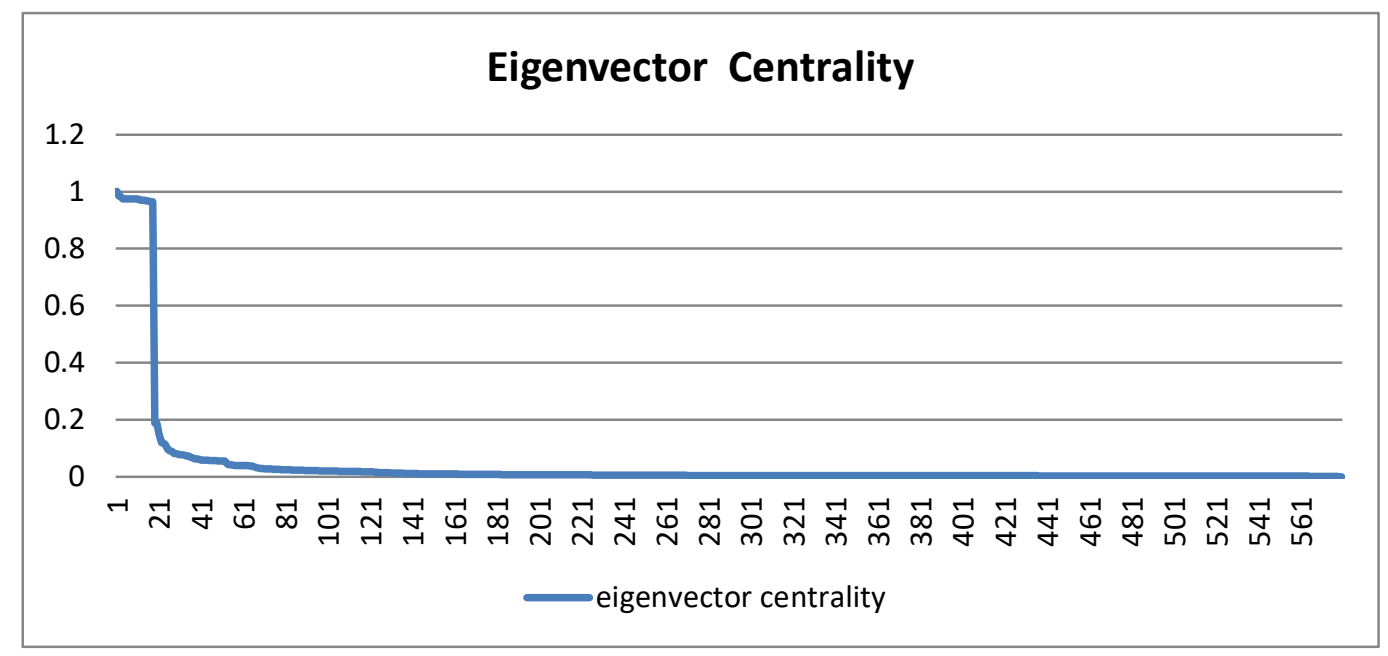




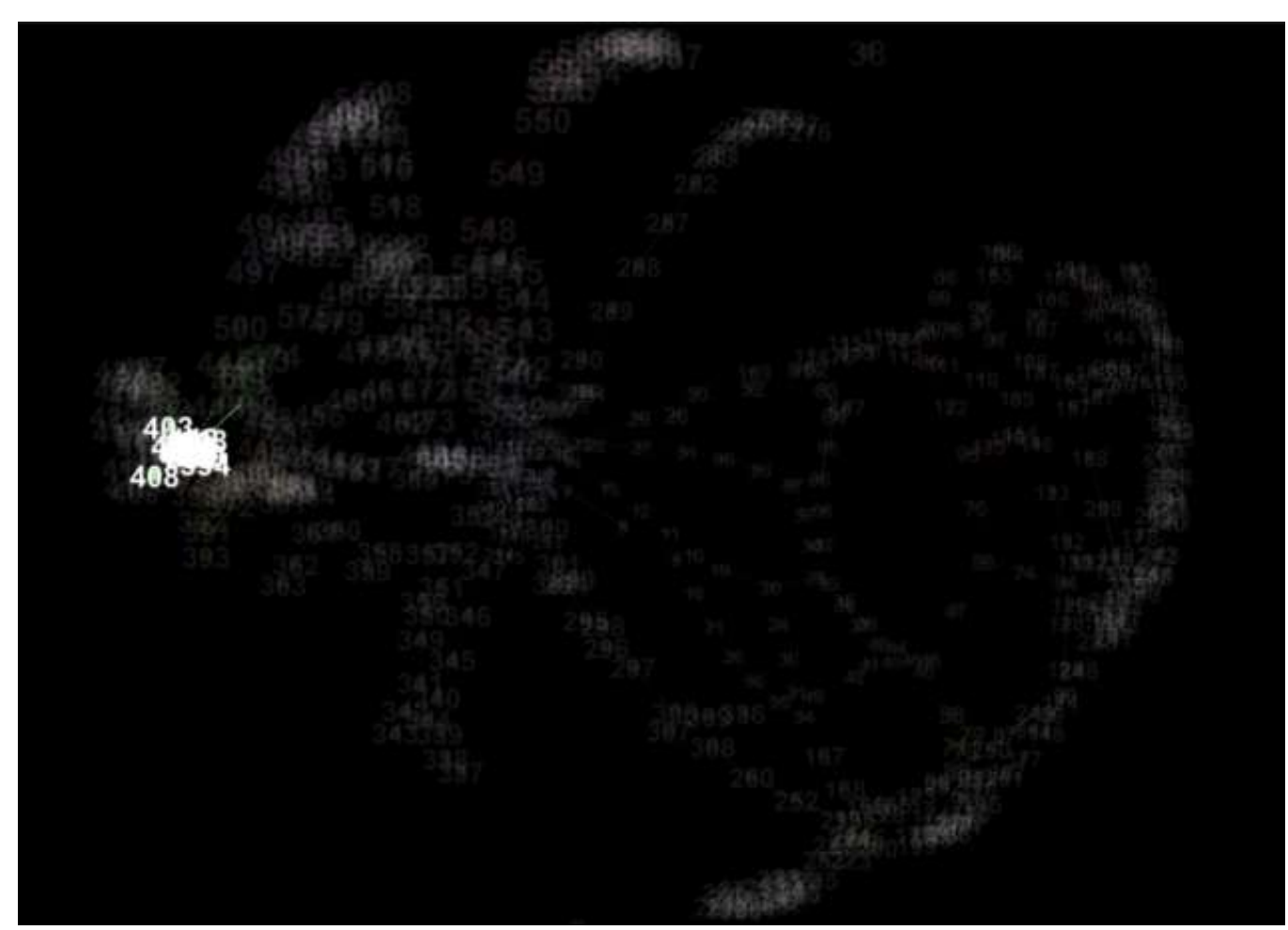

Fig.13 Distribution of nodes with eigenvector centrality number values $>0.9$

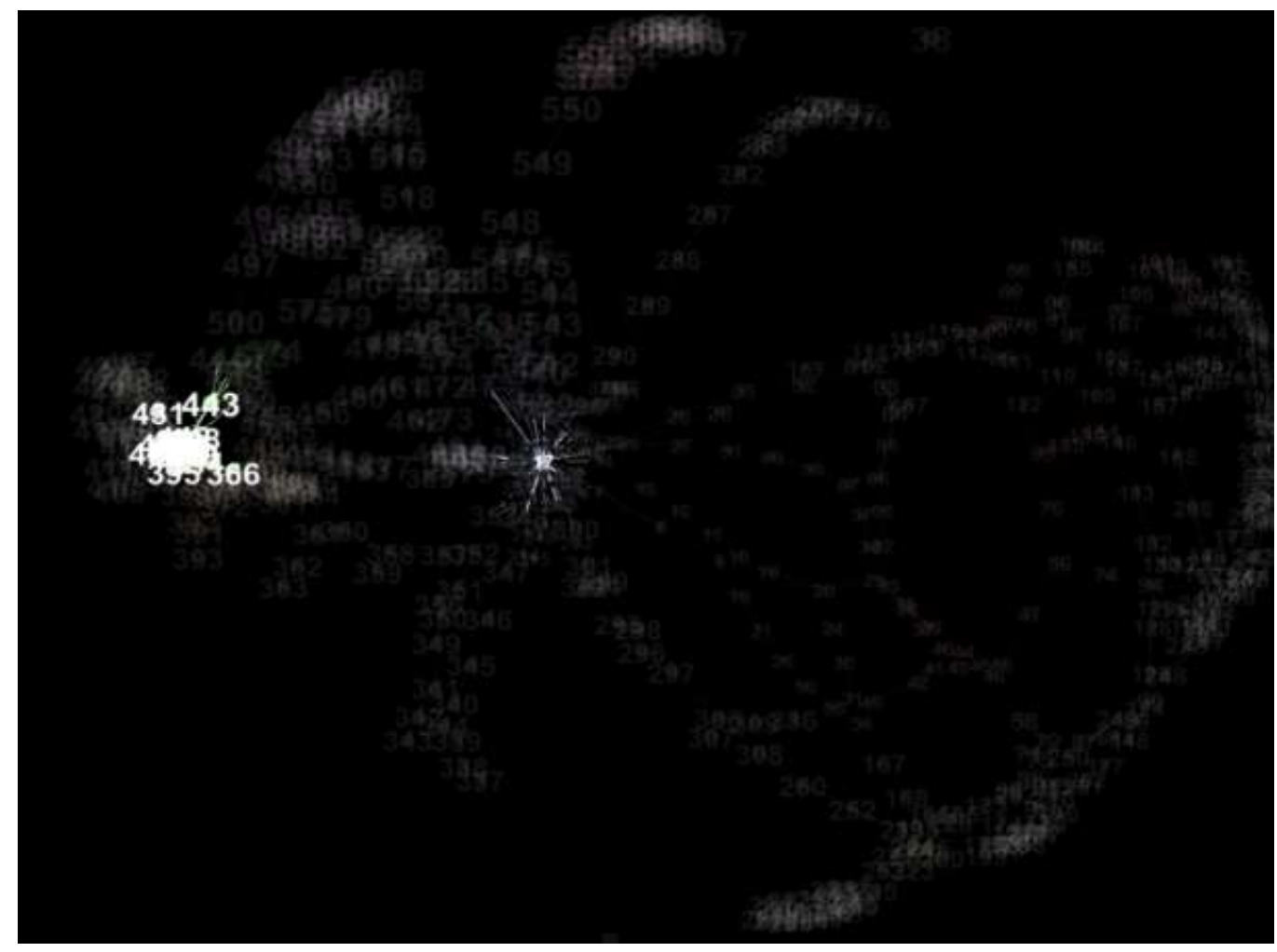

Fig.14 Distribution of nodes with eigenvector centrality number values $>0.1$

(5) Pagerank and Clustering Coefficient 
PageRank is Google's proprietary algorithm for measuring the importance of a particular web page relative to other pages in the search engine index. In complex networks, nodes that can be reached from more places are usually more important and therefore have higher PageRank. (Ben-Naim, 2004) Table 10 and Fig. 15 show that nodes with high PageRank values in the network are divided into two cases. One is such as node 17, its own degree value is high, there are many surrounding water system paths to the node, so the PageRank value is high, becoming the regional core point; the second is such as 135,159,544 nodes, its own degree value and the number of arrival paths is not high, but its node weighted degree and access paths weighted high, as shown in the current site spatial water network that is the storage point water area is larger, connected to the water system The paths are shorter and the calculated PageRank values are higher. Fig. 15 shows that there are 24 nodes (groups) with high PageRank values in this network, which become strategic key points in the water storage network. When flooding occurs, regional flood water is quickly gathered to these storage points through the connected water system, which greatly alleviates the flooding situation. At the same time, according to the regional flood storage volume, the sink area of nodes (groups) with high PageRank and the number and length of connected water systems are optimized and adjusted to control key storage nodes (groups), simplify the network structure, enhance network stability and defensibility, and improve the flood storage function.

In graph theory, the clustering coefficient is a parameter that indicates the degree of aggregation of nodes in a graph. (Noble, 2009) Specifically, it is the degree to which the neighboring points of a point are connected to each other. In Fig. 16, the nodes with an clustering coefficient of 1 have their neighboring nodes connected to each other, forming a cluster of open networks with relatively high density. In the current spatial water network, these nodes with high aggregation coefficients are more evenly distributed throughout the network space, and form multiple clusters with higher densities with neighboring nodes, showing a homogeneous water network on a global macroscopic scale, and a large heterogeneity on a local microscopic scale. This pattern does not form a core key point in the local area, but exists as a group of key points connected to each other. This is conducive to combining the site geography and urban planning and development requirements to flexibly adjust individual nodes without affecting the overall storage transfer capability. Each node in the cluster is replaceable, and the importance of individual nodes is not emphasized, only the integrated storage capacity of the cluster nodes needs to be controlled.

These two parameter algorithms give different meanings to the network nodes, which are analyzed and compared together to facilitate the measurement of the network status and value of the nodes. Based on the quantitative analysis results of node pagerank and clustering coefficient, combined with the current geographical conditions and urban development needs, the node location, area and the number and length of water system paths are appropriately integrated, and the parameter attributes are adjusted and optimized to explore a stable, efficient and synergistic development of flood safety pattern. 
Table 10 Nodal pageranks value table

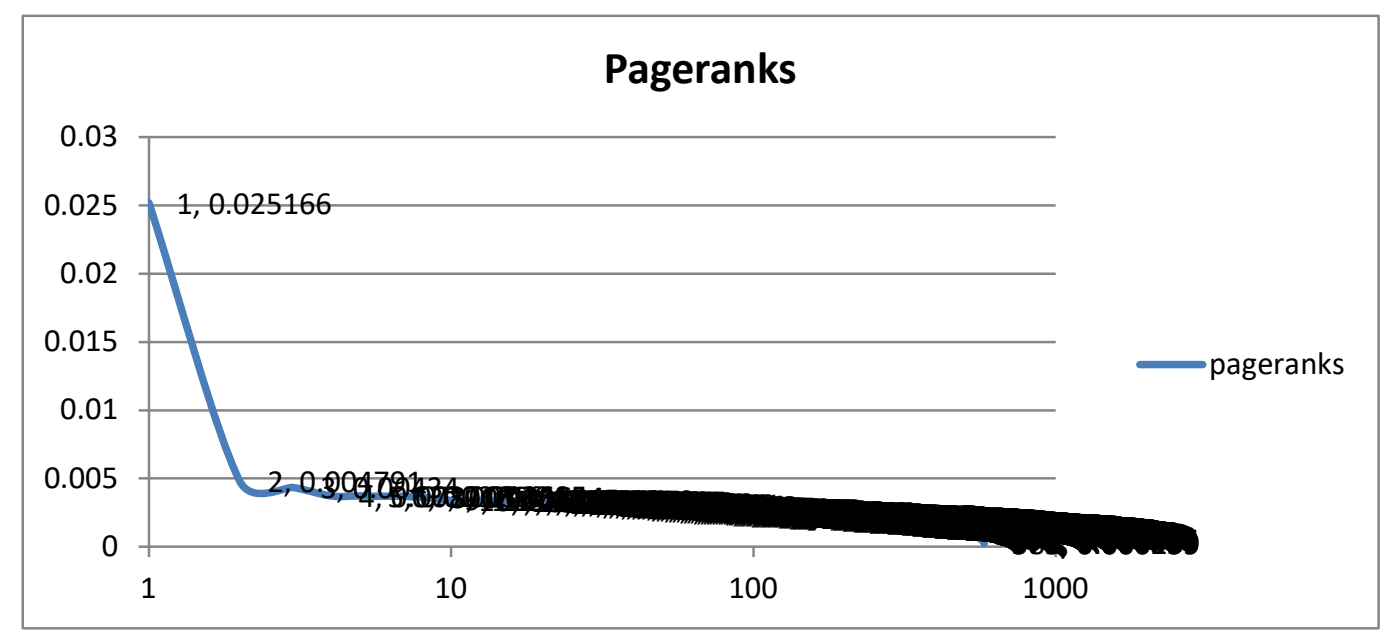

Table 11 Nodal clustering coefficient value table

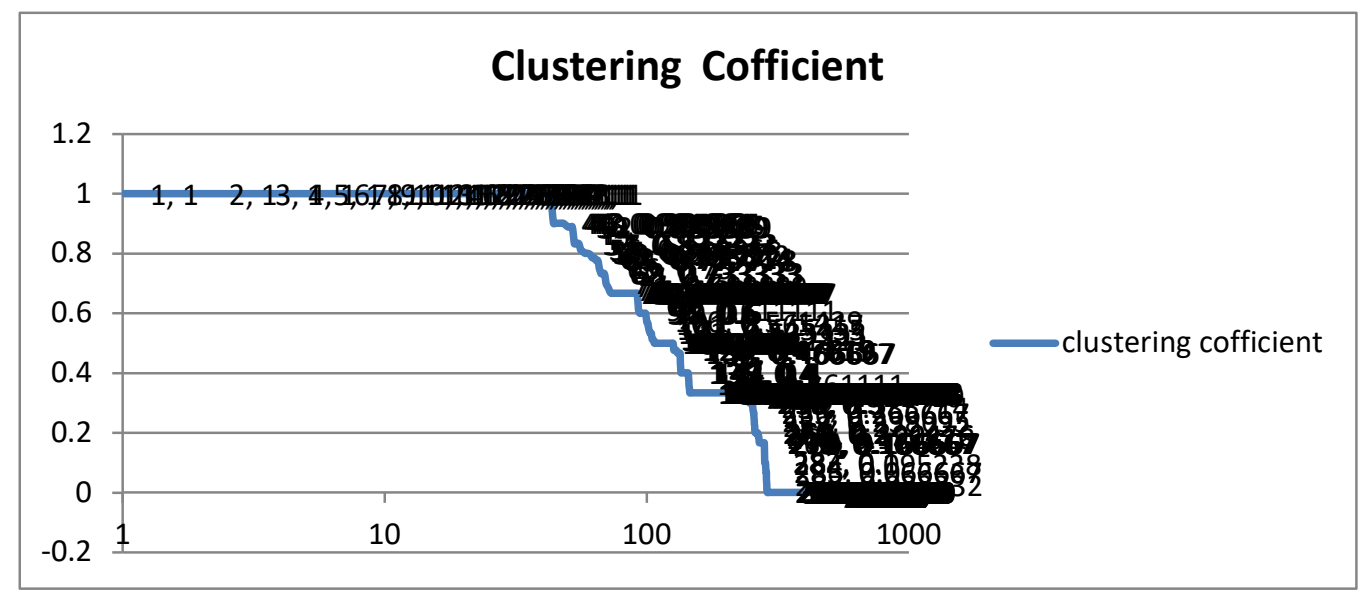




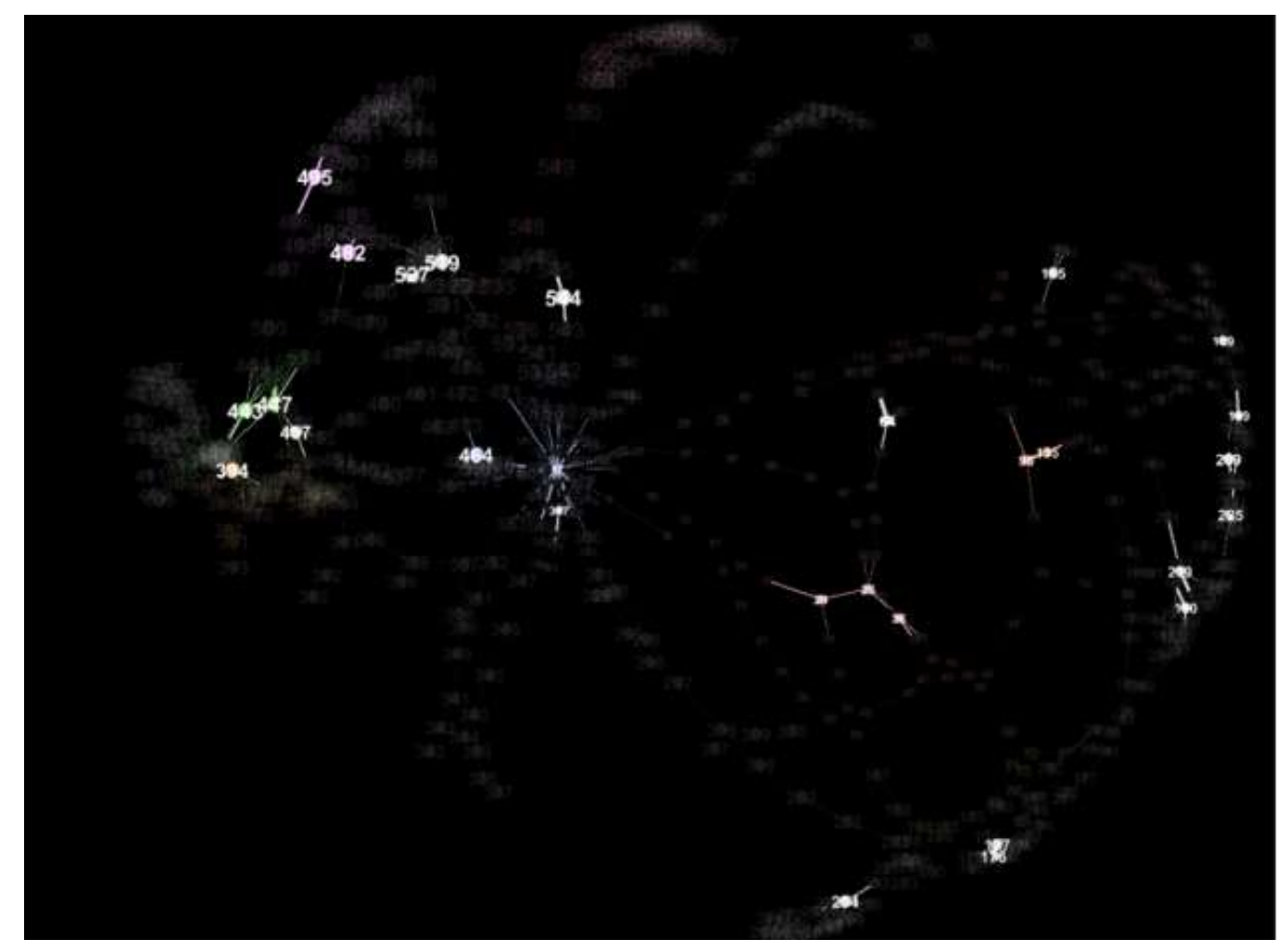

Fig.15 Network distribution of nodes (groups) with higher Pagerank values

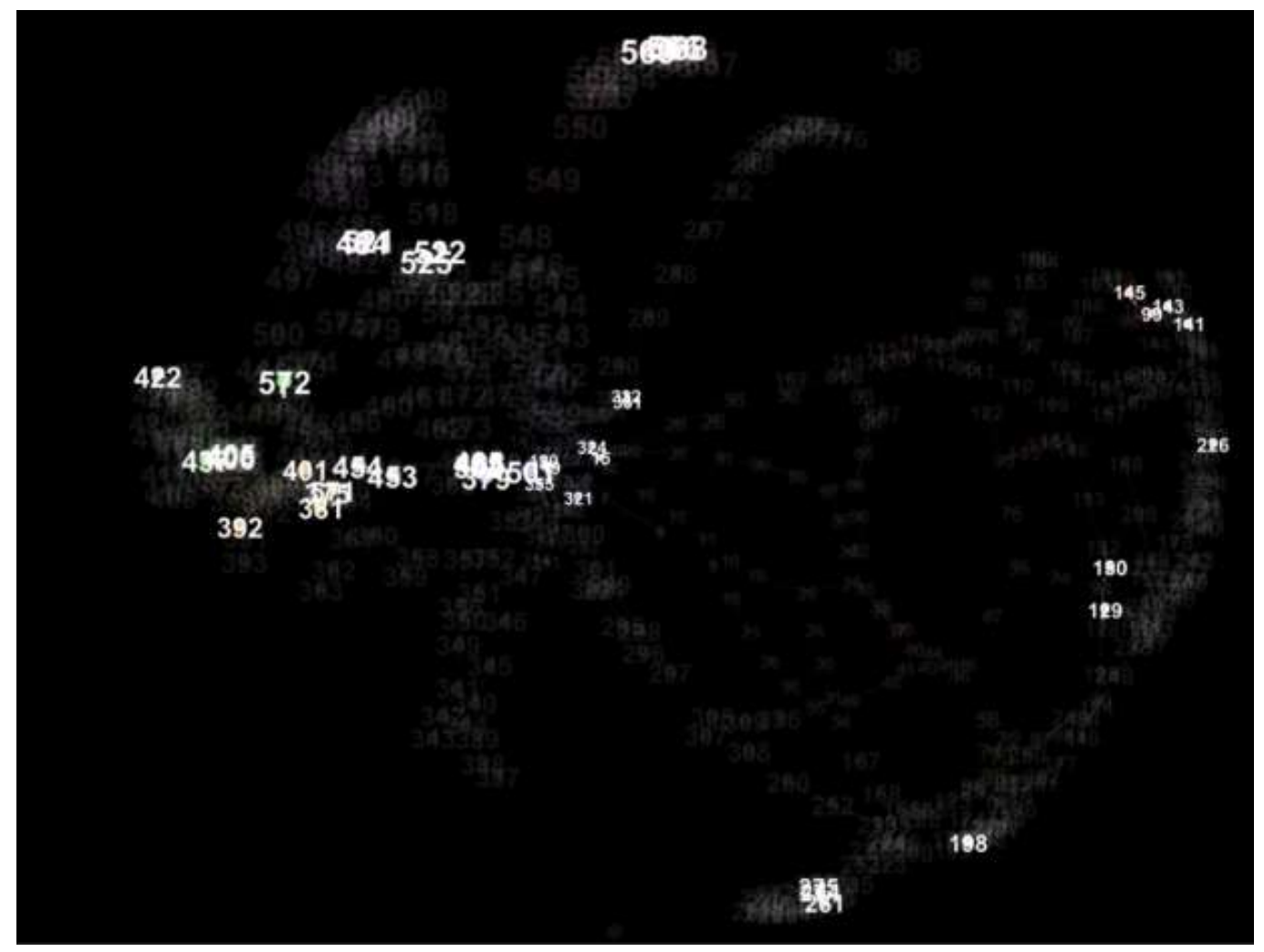

Fig.16 Network distribution of nodes (groups) with clustering coefficient of 1 


\section{(6) modularity_class}

Modularity_class is an important community evaluation metric that utilizes the definition of community structure, where nodes within a community are more closely connected and the probability of two nodes in a community having an edge is higher than the probability of two nodes in a random graph having an edge (Albert R, 2002). The community structure is a reflection of the nature of the network at the mesoscopic scale, and the study of the structure of the communities in the network is an important way to understand the structure and function of the whole network. As shown in Table 12, the whole network is divided into 27 associations, among which there are 4 associations with more than 30 nodes. As shown in Fig.17, with Node 17 (Chao Phraya River) as the core, the community with 57 nodes as the largest association is distributed radially to form the core of the entire network. The other three large societies are located in the eastern, northeastern, and western regions of the Chao Phraya River Society. The eastern society are arranged in a ring shape, the northeastern society are arranged in a tandem shape, and the western society are distributed in a composite shape (both tandem and ring), and these three major societies are the core societies in each region. The 11 major societies with nodes in the range of 20-30 are shown in Fig.18, forming a radial structure that connects the core societies with other end societies and forms the main society structure of the network with the 4 major core societies. Through node modularity_class analysis, a clear network structure and community hierarchy is obtained, which lays the foundation for building a flood safety pattern.

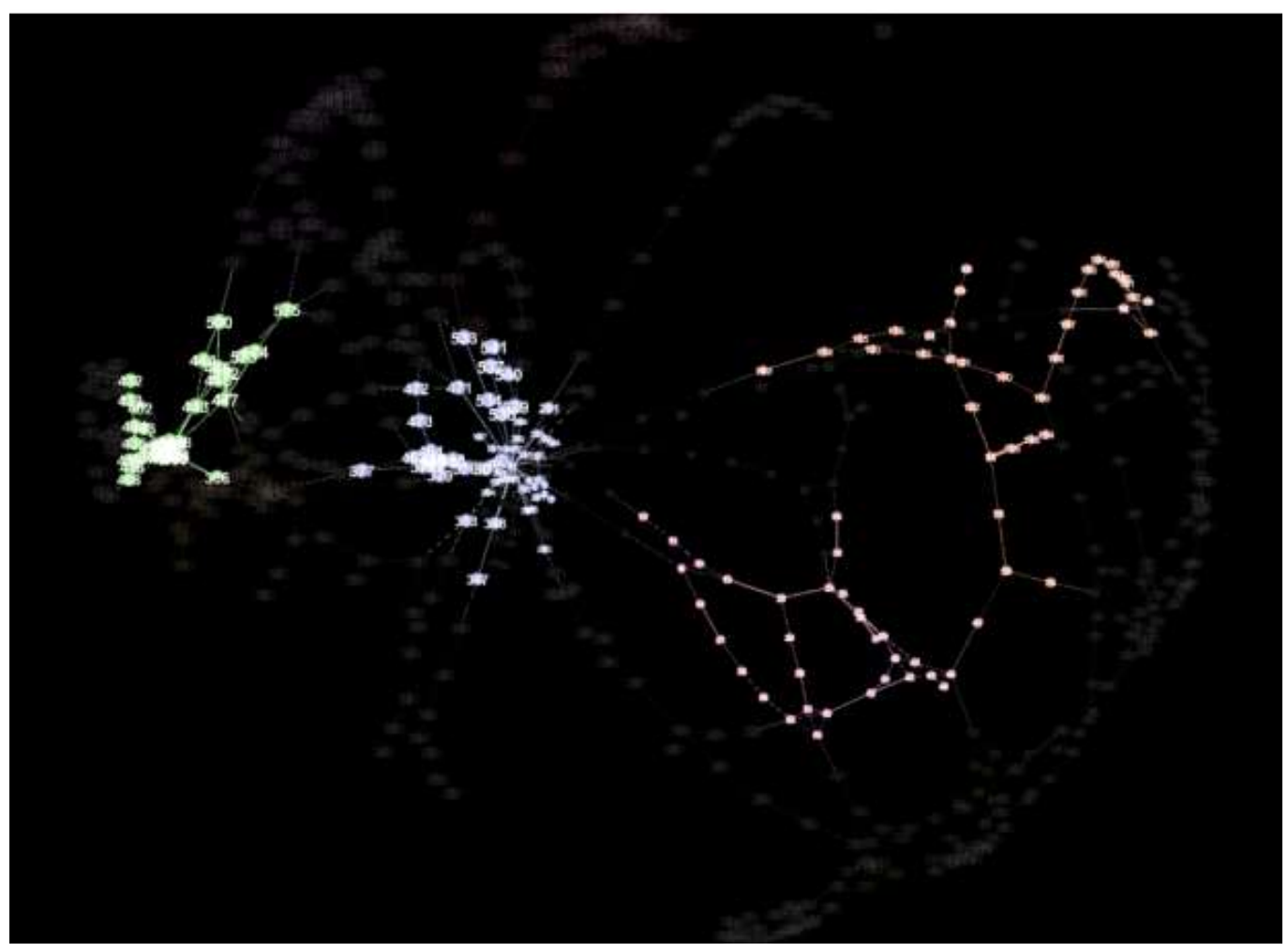

Fig.17 Network distribution of the four core associations 


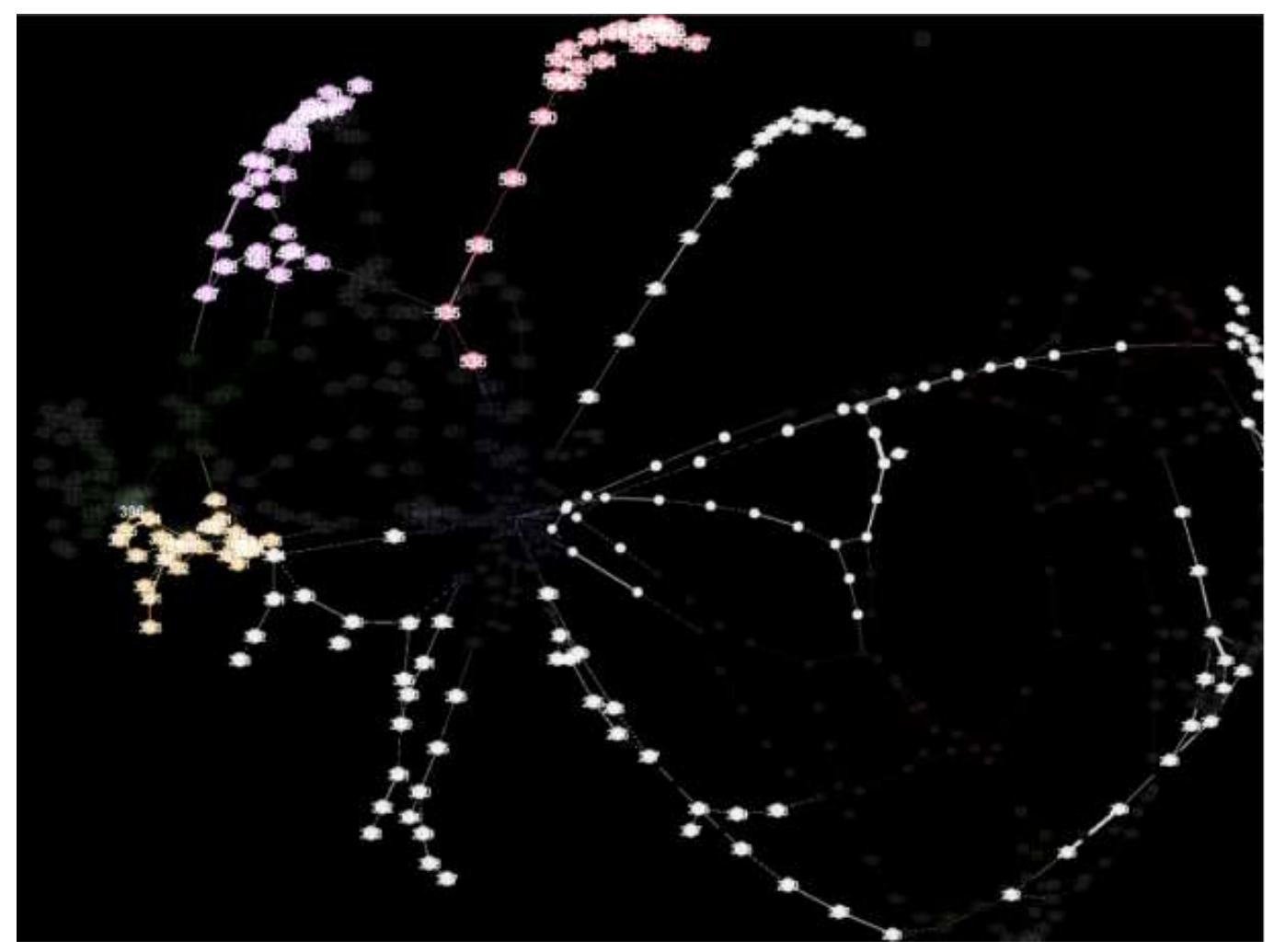

Fig.18 Network distribution of 11 major associations

Table 12 Nodal number distribution of the 27 associations

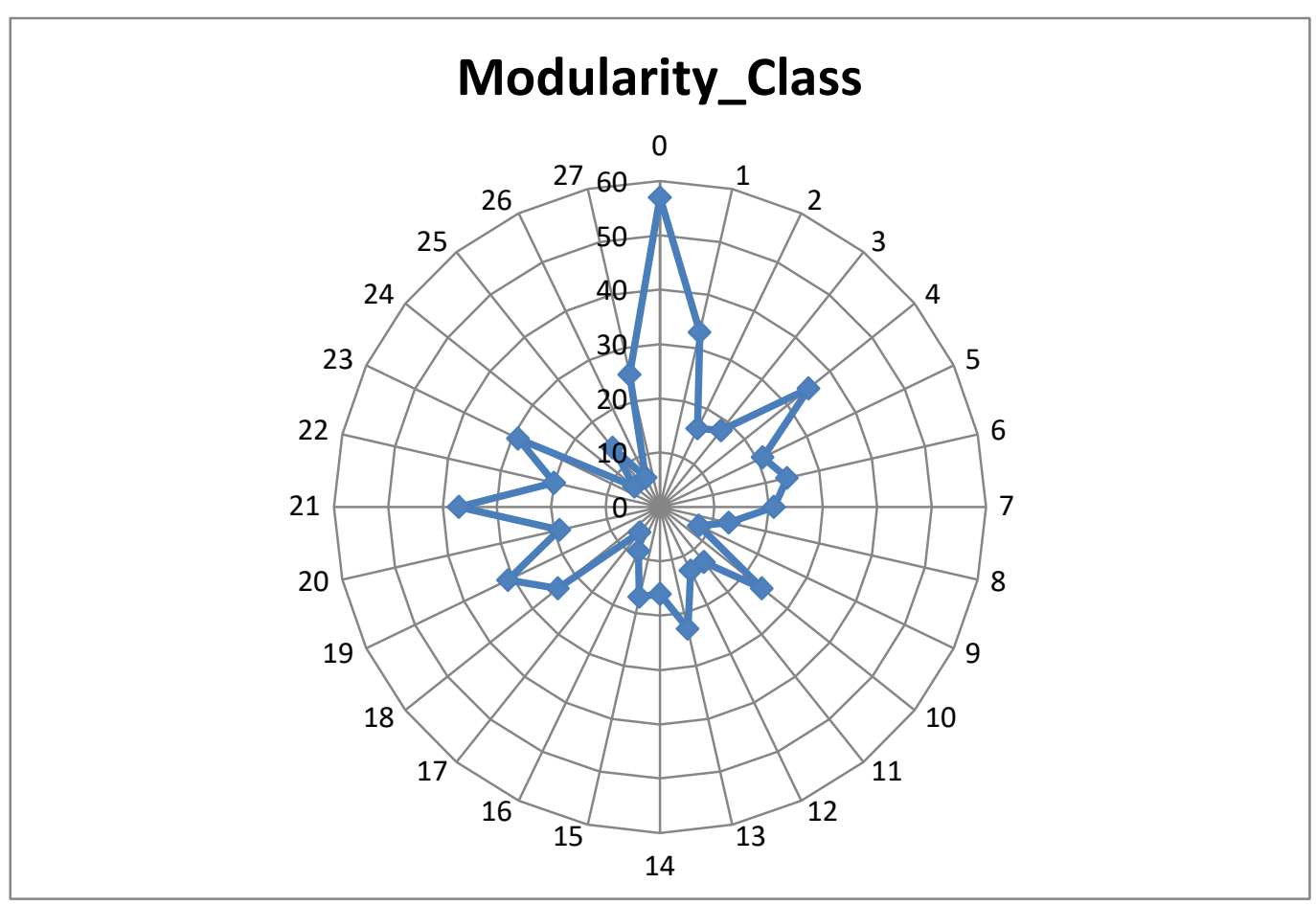

3.2 Network evolution model 
The average degree measures the activity of the overall network nodes, with lower degree values indicating less interaction between nodes and conversely more connections. Using Gephi software, the average degree of the water network in the built-up area of Bangkok is 3.666, indicating that on average each storage point is connected to 3.7 surrounding storage points. In a water network with a planar spatial layout, this connectivity degree is high, indicating that the Bangkok water system network has a good basis for flood storage. The average weighted degree is calculated as the node degree based on the weights of connected edges, and then the average degree is calculated based on the weighted degree, which is the sum of the weighted degrees of all nodes divided by the number of nodes. The average degree is a special case of the average weighted degree, and the weight of each edge of the average degree is 1 . The value of the weight of the edge in this water network is defined as 100 times the inverse of the length of the water system path, and the average weighted degree of the water system storage network is calculated as 100.232 with a weighted degree of 27.34. The length of the edge of the entire network weighted path is 0.4 $\mathrm{km}$, which coincides with the scale of a small modern urban neighborhood $(0.4 \mathrm{~km} \times 0.4 \mathrm{~km})$.

The diameter of this water system network is 31, and based on the average water system length of $1.5 \mathrm{~km}$ between the transfer points (the total length of 1063 path sides is $1589.5 \mathrm{~km}$ ), the length of the network diameter is $46.5 \mathrm{~km}$, which is consistent with the distance between the farthest east and west points of the built-up area of Bangkok. The network graph density is 0.006 , which indicates that the network nodes are loosely connected and belong to a sparse network. This is because the distance between water network storage points is limited by flat space, unlike virtual networks such as the Internet, interpersonal networks, and social networks, which are not limited by spatial distance and will have a high degree of closeness. The lower graph density is a characteristic of geospatial physical networks.

Network modularity is used to measure whether the division of a community is a relatively good result. A relatively good result has a high similarity of nodes inside the community and a low similarity of nodes outside the community. The magnitude of modularity is defined as the ratio of the total number of edges inside the community to the total number of edges in the network minus an expectation value. This expectation value is the magnitude of the ratio of the total number of edges within the community formed by the same community assignment to the total number of edges in the network when the network is set as a random network (Watts D J, 1998). The degree of modularity is expressed as a $\mathrm{Q}$-value ranging from 0 to 1 . A larger $\mathrm{Q}$-value indicates a more accurate community structure for network partitioning. In real networks, the highest $Q$ values generally occur between 0.3 and 0.7. The modularity of this water system network is 0.899 , which is a high value, indicating that the community structure level of the whole water system network is very obvious, and further analysis of each community structure level and key storage points has important practical significance and basis for the layout planning of the water network geospatially.

The clustering coefficient describes the likelihood that neighboring nodes of individuals in the network are also neighbors of each other. In general, the magnitude of the clustering coefficient affects the propagation dynamics on the network, and the larger the average node 
clustering coefficient, the slower the propagation when other parameters are constant (Watts D J, 1998). The average clustering coefficient of 0.28 for this network is a low value, indicating that the connectivity between a storage point and its surrounding storage points is not tight, which is limited by the characteristics of the planar geospatial network. The low clustering coefficient ensures the timely spatial transport and diffusion of the stored water, which facilitates the rapid deployment of inter-regional flooding.

The eigenvector centrality of this water network is 0.032 , and the network tends to be randomized. Combined with the node degree parameter distribution, there are a few important key points in the network in the absolute core position, making the network again has scale-free network characteristics. Using the robustness characteristics of the scale-free network to occupy key storage points and important water system paths is important for rapid urban flood discharge and flooding.

The average path length of the water network is 12.466. Based on the average water system length of $1.5 \mathrm{~km}$ between the storage points, the average path length of the network is $18.7 \mathrm{~km}$, which is slightly smaller than the diameter of the core built-up area of Bangkok (20 $\mathrm{km}$ ), indicating that the constructed network conforms to the current geospatial characteristics. For planar spatial networks such as geoscape and water system storage networks, identifying the key locations, nodes and paths in their networks is an important purpose of studying the networks. By identifying these key nodes and important paths to simplify the network structure and optimize the spatial layout, the scientific and quantitative design of geoscape and water system storage network is realized.

In summary, the constructed water system storage network is a typical flat geospatial network, which is different from the Internet, social networks, interpersonal networks, communication networks and other networks that are not constrained by space. Through the verification of each characteristic parameter, the simulated constructed water system storage network is consistent with the characteristics of complex networks and can be scientifically quantified and analyzed. This lays the foundation for the planning, layout and optimization of the water system storage network in the built-up area of Bangkok by using the theory related to complex networks, and introduces a new research method for the analysis, planning and evaluation of urban water networks. The evolution model of water system storage network constructed by the simulation is different from the common single rule network model, stochastic network model, small world network model or scale-free network model in complex networks, but presents a composite network model feature. Due to the influence of planar geospatial factors, its network has the characteristics of a partially random network model, with lower graph density, average clustering coefficient and eigenvector centrality than the small-world network and regular network models, and larger network diameter and average path length than the small-world network model and regular network model. And its node degree distribution is similar to the scale-free network model, with typical robustness and vulnerability; meanwhile, the network modularity value is high, the association structure is obvious, and some associations have self-similar network model characteristics. The analysis of these characteristics introduces a new field for the study of water system storage networks.

3.3 Flood safety pattern in the built-up area of Bangkok 
Based on the water storage network map of the built-up area of Bangkok (Fig.19), the attribute parameters of 581 network nodes were analyzed using complex network correlation theory. As shown in Tables 13 and 14, the green color indicates that the importance of nodes is measured by five attribute parameters such as degree, authority, hub, eigenvector centrality and triangles, and the nodes with the top ranking are selected as the key planning and construction nodes; the magenta color indicates that the important connectivity and sparsity of nodes are measured by three parameters such as Closeness Centrality, Harmonic closness centrality, Betweeness centrality, etc. The nodes with the top ranking of parameters are selected as the key planning and construction nodes; the purple and orange colors indicate that the centrality of nodes is measured by two parameters reflecting the degree of aggregation, pagerank and clustering coefficient, and the nodes with the top ranking of the parameters are selected as the key planning and construction nodes. Through the ranking statistics of 581 storage points with 11 parameters in 4 categories, 120 storage points with more than 6 parameters ranked in the top 100 were selected as the key nodes of the network (shown in Table 14), combined with 3 parameters, such as Weight, Weighted degree and Modularity, which reflect the catchment area of storage points and the structural characteristics of network associations, and Based on the full consideration of the water network and the surrounding geographic space, urban environment, road traffic and planning and construction, 25 key nodes were added, and a total of 145 key storage points were identified. By sorting out and planning these important nodes and their important connecting paths, a flood safety pattern of Bangkok built-up area with 145 key nodes and 127 important rivers as the backbone was constructed (shown in Fig.20), and the robustness and vulnerability theories in complex networks were also applied to the tolerance and resistance of the flood safety pattern.

The flood safety pattern in the built-up area of Bangkok is based on a framework of radioactive backbone cluster points, linking major associations and forming wedge-shaped open spaces penetrating into the core area of the city in combination with the water system path. In the core area, small storage points are built in combination with urban green space and open space to save land for urban development as much as possible. Large-scale storage points are built at key nodes where the surrounding land is more relaxed as the kidney of the city and used as the main storage site for urban flooding. In the absence of flooding in the upper reaches of the Chao Phraya River, when only the Bangkok area is flooded by rainfall, the core built-up area with a radius of $10 \mathrm{~km}$ is mainly for drainage and supplemented by storage, while the west bank and other peripheral areas are mainly for storage and supplemented by drainage. If flooding occurs in the upper reaches of the Chao Phraya River and backflow occurs in Bangkok downstream, the core built-up area is mainly drained and water is quickly transported to storage sites in peripheral areas through the backbone cluster water network to ensure maximum flood safety in the core city. 


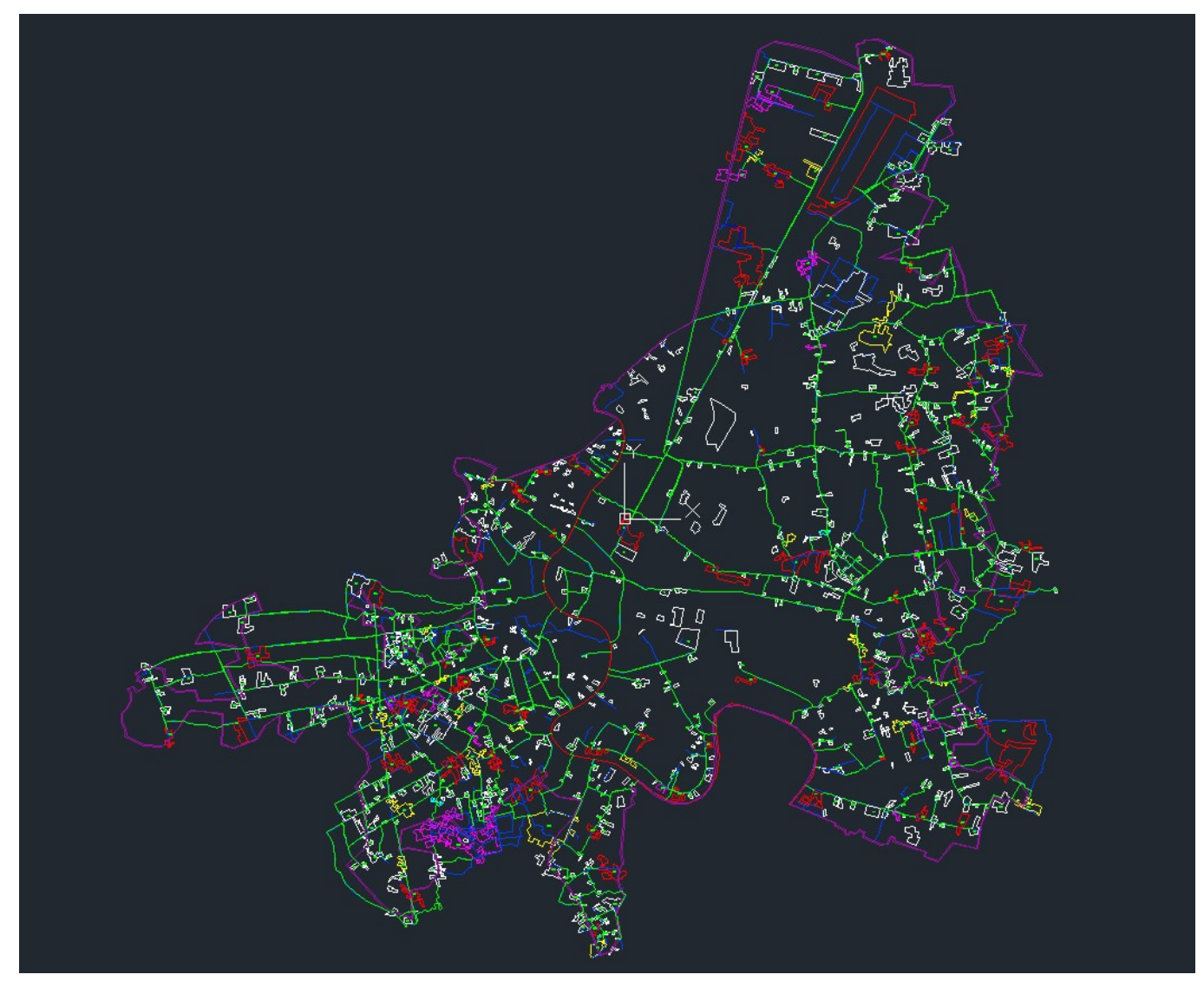

Fig.19 Map of the water storage network in the built-up area of Bangkok 
Table 13 Network node parameter ranking table

\begin{tabular}{|c|c|c|c|c|c|c|c|c|c|c|c|c|}
\hline $\begin{array}{l}\text { Id } \\
\text { (weight } \\
\text { ) }\end{array}$ & $\begin{array}{c}\text { Id } \\
\text { (degree } \\
\text { ) }\end{array}$ & $\begin{array}{c}\mathrm{Id} \\
\text { (weighte } \\
\mathrm{d} \\
\text { degree) } \\
\end{array}$ & $\begin{array}{c}\text { Id (closne } \\
\text { sscentral } \\
\text { ity) }\end{array}$ & $\begin{array}{c}\text { Id (harmon } \\
\text { icclosnes } \\
\text { scentrali } \\
\text { ty) }\end{array}$ & $\begin{array}{c}\text { Id (betwee } \\
\text { nesscentr } \\
\text { ality) }\end{array}$ & $\begin{array}{c}\text { Id(modula } \\
\text { rity_clas } \\
\text { s) }\end{array}$ & $\begin{array}{c}\text { Id (Author } \\
\text { ity) }\end{array}$ & Id (Hub) & $\begin{array}{c}\text { Id (pagera } \\
\text { nks) }\end{array}$ & $\begin{array}{c}\text { Id (cluste } \\
\text { ring) }\end{array}$ & $\begin{array}{c}\text { Id (triang } \\
\text { les) }\end{array}$ & $\begin{array}{c}\text { Id (eigenc } \\
\text { entrality } \\
\text { ) }\end{array}$ \\
\hline 100 & 17 & 17 & 17 & 17 & 17 & 17 & 394 & 394 & 17 & 465 & 394 & 394 \\
\hline 17 & 394 & 447 & 22 & 471 & 364 & 470 & 442 & 442 & 254 & 572 & 413 & 413 \\
\hline 259 & 413 & 159 & 23 & 365 & 365 & 468 & 413 & 413 & 447 & 355 & 442 & 442 \\
\hline 98 & 417 & 394 & 365 & 22 & 22 & 378 & 396 & 396 & 219 & 566 & 441 & 396 \\
\hline 519 & 396 & 64 & 471 & 470 & 471 & 463 & 369 & 369 & 135 & 484 & 440 & 369 \\
\hline 535 & 436 & 219 & 300 & 468 & 28 & 464 & 368 & 368 & 64 & 145 & 369 & 368 \\
\hline 76 & 442 & 443 & 14 & 23 & 52 & 465 & 367 & 367 & 394 & 275 & 368 & 367 \\
\hline 164 & 439 & 544 & 16 & 14 & 62 & 466 & 441 & 441 & 464 & 437 & 367 & 441 \\
\hline 575 & 367 & 160 & 7 & 16 & 532 & 502 & 440 & 440 & 519 & 405 & 396 & 440 \\
\hline 173 & 407 & 495 & 15 & 576 & 300 & 5 & 439 & 439 & 527 & 454 & 438 & 439 \\
\hline 184 & 448 & 434 & 534 & 15 & 306 & 576 & 436 & 436 & 155 & 143 & 439 & 436 \\
\hline 25 & 369 & 135 & 5 & 7 & 297 & 325 & 438 & 438 & 482 & 406 & 436 & 417 \\
\hline 186 & 368 & 457 & 6 & 534 & 299 & 323 & 417 & 417 & 225 & 375 & 405 & 448 \\
\hline 133 & 441 & 396 & 470 & 353 & 298 & 471 & 448 & 448 & 95 & 332 & 406 & 438 \\
\hline 532 & 404 & 254 & 468 & 300 & 519 & 320 & 407 & 407 & 25 & 331 & 404 & 407 \\
\hline 358 & 438 & 572 & 576 & 5 & 79 & 354 & 404 & 404 & 159 & 466 & 448 & 404 \\
\hline 357 & 440 & 564 & 538 & 364 & 308 & 353 & 405 & 405 & 443 & 129 & 407 & 405 \\
\hline 310 & 406 & 430 & 291 & 394 & 260 & 292 & 406 & 406 & 127 & 381 & 417 & 406 \\
\hline 447 & 405 & 395 & 353 & 325 & 80 & 305 & 366 & 366 & 544 & 130 & 384 & 366 \\
\hline 16 & 384 & 180 & 348 & 6 & 98 & 501 & 443 & 443 & 209 & 522 & 383 & 443 \\
\hline 189 & 519 & 397 & 325 & 317 & 252 & 355 & 395 & 395 & 495 & 120 & 382 & 17 \\
\hline 552 & 386 & 527 & 539 & 323 & 233 & 2 & 431 & 431 & 317 & 525 & 380 & 395 \\
\hline 367 & 378 & 95 & 354 & 320 & 81 & 539 & 437 & 437 & 457 & 401 & 386 & 431 \\
\hline 482 & 383 & 566 & 501 & 539 & 82 & 120 & 373 & 373 & 36 & 521 & 375 & 437 \\
\hline 225 & 447 & 217 & 317 & 354 & 366 & 119 & 389 & 389 & 189 & 198 & 571 & 373 \\
\hline 306 & 374 & 545 & 323 & 312 & 7 & 377 & 390 & 390 & 176 & 226 & 376 & 389 \\
\hline 12 & 376 & 563 & 292 & 538 & 8 & 4 & 388 & 388 & 20 & 261 & 374 & 447 \\
\hline 443 & 382 & 63 & 305 & 292 & 9 & 534 & 447 & 447 & 180 & 568 & 378 & 390 \\
\hline 366 & 450 & 519 & 328 & 305 & 20 & 328 & 414 & 414 & 434 & 141 & 17 & 388 \\
\hline 246 & 463 & 559 & 320 & 291 & 25 & 538 & 416 & 416 & 42 & 392 & 470 & 414 \\
\hline 156 & 380 & 139 & 312 & 311 & 535 & 348 & 424 & 424 & 279 & 502 & 468 & 416 \\
\hline 139 & 100 & 438 & 311 & 348 & 19 & 291 & 412 & 412 & 396 & 274 & 463 & 424 \\
\hline 497 & 464 & 482 & 2 & 328 & 46 & 303 & 370 & 370 & 306 & 15 & 465 & 412 \\
\hline 187 & 468 & 429 & 4 & 2 & 23 & 121 & 397 & 397 & 340 & 324 & 466 & 384 \\
\hline 413 & 571 & 150 & 303 & 501 & 29 & 333 & 434 & 434 & 217 & 453 & 502 & 370 \\
\hline 6 & 470 & 458 & 121 & 4 & 53 & 117 & 435 & 435 & 190 & 569 & 464 & 386 \\
\hline 480 & 375 & 218 & 355 & 303 & 169 & 577 & 408 & 408 & 102 & 571 & 450 & 397 \\
\hline 185 & 535 & 496 & 120 & 121 & 548 & 118 & 403 & 403 & 430 & 422 & 385 & 383 \\
\hline 503 & 173 & 464 & 119 & 355 & 114 & 469 & 419 & 419 & 215 & 119 & 16 & 434 \\
\hline 191 & 189 & 250 & 333 & 120 & 549 & 319 & 418 & 418 & 150 & 379 & 212 & 435 \\
\hline 491 & 482 & 441 & 117 & 119 & 232 & 334 & 445 & 445 & 535 & 321 & 213 & 374 \\
\hline 254 & 443 & 249 & 577 & 333 & 550 & 3 & 446 & 446 & 465 & 99 & 401 & 376 \\
\hline 417 & 366 & 36 & 118 & 117 & 39 & 379 & 364 & \begin{tabular}{|l|l|}
364 \\
\end{tabular} & 358 & 501 & 15 & 382 \\
\hline 262 & 94 & 197 & 469 & 577 & 228 & 472 & 371 & 371 & 348 & 441 & 400 & 380 \\
\hline 548 & 485 & 513 & 319 & 118 & 193 & 533 & 444 & 444 & 243 & 440 & 23 & 408 \\
\hline 518 & 211 & 269 & 334 & 469 & 44 & 322 & 415 & 415 & 73 & 369 & 14 & 403 \\
\hline 137 & 364 & 494 & 28 & 319 & 144 & 293 & 430 & 430 & 160 & 368 & 210 & 419 \\
\hline 236 & 193 & 450 & 299 & 334 & 113 & 473 & 428 & 428 & 220 & 367 & 449 & 418 \\
\hline 423 & 385 & 455 & 364 & 369 & 76 & 324 & 573 & 573 & 134 & 438 & 189 & 375 \\
\hline 215 & 373 & 435 & 532 & 368 & 112 & 1 & 572 & 572 & 22 & 404 & 22 & 571 \\
\hline 261 & 22 & 65 & 8 & 367 & 164 & 540 & 372 & 372 & 252 & 448 & 211 & 470 \\
\hline 96 & 466 & 439 & 29 & 373 & 222 & 537 & 387 & 387 & 211 & 407 & 193 & 468 \\
\hline 457 & 465 & 512 & 27 & 532 & 77 & 321 & 386 & 386 & 63 & 382 & 519 & 378 \\
\hline 568 & 502 & 209 & 533 & 448 & 520 & 541 & 385 & 385 & 259 & 380 & 100 & 463 \\
\hline 148 & 98 & 25 & 301 & 366 & 78 & 347 & 449 & 449 & 513 & 526 & 446 & 16 \\
\hline 240 & 25 & 181 & 13 & 413 & 373 & 304 & 420 & 420 & 86 & 442 & 445 & 22 \\
\hline 97 & 16 & 390 & 472 & 378 & 291 & 147 & 391 & 391 & 572 & 396 & 414 & 464 \\
\hline 394 & 139 & 225 & 474 & 463 & 290 & 44 & 392 & 392 & 269 & 439 & 447 & 23 \\
\hline 411 & 491 & 440 & 463 & 442 & 58 & 43 & 457 & 457 & 282 & 436 & 416 & 14 \\
\hline 203 & 254 & 388 & 378 & 439 & 223 & 46 & 425 & 425 & 545 & 446 & 524 & 385 \\
\hline 99 & 215 & 127 & 537 & 441 & 94 & 40 & 574 & 574 & 395 & \begin{tabular}{|l|}
417 \\
\end{tabular} & 390 & 465 \\
\hline 291 & 414 & 391 & 293 & 440 & 59 & 25 & 411 & 411 & 295 & 470 & 140 & 466 \\
\hline 556 & 140 & 561 & 357 & 396 & 97 & 55 & 398 & 398 & 559 & 468 & 389 & 502 \\
\hline 207 & 14 & 560 & 298 & 436 & 289 & 39 & 433 & 433 & 250 & 376 & 482 & 15 \\
\hline 37 & 445 & 543 & 464 & 417 & 235 & 45 & 409 & 409 & 76 & 374 & 173 & 450 \\
\hline 198 & 213 & 189 & 540 & 407 & 394 & 49 & 402 & 402 & 128 & 464 & 485 & 364 \\
\hline 414 & 390 & 176 & 465 & 374 & 137 & 36 & 500 & 500 & 139 & 16 & 373 & 449 \\
\hline 175 & 212 & 243 & 466 & 404 & 250 & 54 & 374 & 374 & 563 & 212 & 572 & 6 \\
\hline 455 & 127 & 448 & 502 & 519 & 485 & 31 & 384 & 384 & 455 & 213 & 484 & 445 \\
\hline 153 & 210 & 524 & 52 & 438 & 288 & 35 & 429 & 429 & 438 & 416 & 521 & 5 \\
\hline 419 & 524 & 437 & 379 & 405 & 211 & 9 & 450 & 450 & 245 & 383 & 141 & 576 \\
\hline 288 & 23 & 270 & 9 & 406 & 249 & 42 & 361 & 361 & 98 & 413 & 388 & 446 \\
\hline 94 & 389 & 220 & 3 & 389 & 413 & 20 & 360 & 360 & 94 & 176 & 424 & 7 \\
\hline 514 & 400 & 190 & 1 & 472 & 248 & 19 & 365 & 365 & 458 & 395 & 412 & 400 \\
\hline 277 & 449 & 20 & 290 & 533 & 47 & 10 & 400 & 400 & 165 & 415 & 573 & 401 \\
\hline
\end{tabular}




\begin{tabular}{|c|c|c|c|c|c|c|c|c|c|c|c|c|}
\hline 485 & 73 & 465 & 542 & 464 & 157 & 37 & 401 & 401 & 397 & 400 & 138 & 325 \\
\hline 464 & 259 & 237 & 352 & 474 & 287 & 41 & 383 & 383 & 450 & 292 & 520 & 323 \\
\hline 309 & 575 & 245 & 347 & 384 & 374 & 47 & 382 & 382 & 197 & 142 & 139 & 471 \\
\hline 396 & 532 & 436 & 373 & 386 & 100 & 32 & 380 & 380 & 72 & 35 & 491 & 320 \\
\hline 276 & 503 & 384 & 326 & 465 & 109 & 50 & 376 & 376 & 218 & 23 & 443 & 354 \\
\hline 481 & 455 & 268 & 327 & 466 & 518 & 30 & 375 & 375 & 249 & 61 & 526 & 353 \\
\hline 147 & 131 & 387 & 322 & 502 & 447 & 18 & 571 & 571 & 512 & 14 & 44 & 519 \\
\hline 235 & 412 & 259 & 324 & 387 & 225 & 24 & 575 & 575 & 429 & 506 & 127 & 317 \\
\hline 256 & 505 & 404 & 316 & 390 & 111 & 56 & 432 & 432 & 194 & 523 & 366 & 292 \\
\hline 134 & 326 & 500 & 366 & 535 & 200 & 12 & 410 & 410 & 391 & 210 & 176 & 305 \\
\hline 20 & 527 & 208 & 313 & 388 & 36 & 11 & 427 & 427 & 256 & 6 & 506 & 312 \\
\hline 211 & 102 & 165 & 321 & 28 & 282 & 26 & 426 & 426 & 264 & 354 & 6 & 311 \\
\hline 131 & 517 & 535 & 318 & 447 & 219 & 21 & 455 & 455 & 181 & 244 & 244 & 365 \\
\hline 275 & 416 & 42 & 310 & 357 & 534 & 33 & 421 & 421 & 206 & 516 & 527 & 501 \\
\hline 334 & 424 & 383 & 304 & 377 & 110 & 48 & 458 & 458 & 425 & 449 & 245 & 355 \\
\hline 456 & 138 & 317 & 147 & 537 & 74 & 16 & 423 & 423 & 355 & 91 & 43 & 2 \\
\hline 353 & 245 & 445 & 519 & 8 & 175 & 22 & 399 & 399 & 65 & 305 & 131 & 539 \\
\hline 467 & 126 & 86 & 374 & 376 & 215 & 23 & 393 & 393 & 476 & 463 & 172 & 300 \\
\hline 265 & 62 & 134 & 387 & 299 & 533 & 14 & 381 & 381 & 524 & 388 & 222 & 120 \\
\hline 205 & 520 & 530 & 535 & 379 & 188 & 15 & 497 & 497 & 564 & 424 & 517 & 119 \\
\hline 83 & 222 & 256 & 12 & 383 & 35 & 6 & 451 & 451 & 384 & 412 & 532 & 377 \\
\hline 140 & 474 & 400 & 528 & 27 & 259 & 7 & 17 & 17 & 383 & 573 & 215 & 4 \\
\hline 509 & 85 & 529 & 297 & 29 & 208 & 28 & 358 & 358 & 265 & 138 & 73 & 534 \\
\hline 157 & 55 & 73 & 360 & 13 & 369 & 27 & 362 & 362 & 341 & 520 & 535 & 381 \\
\hline 223 & 15 & 405 & 53 & 540 & 368 & 29 & 377 & 377 & 390 & 385 & 94 & 328 \\
\hline 93 & 40 & 136 & 51 & 528 & 367 & 13 & 482 & 482 & 452 & 394 & 332 & 538 \\
\hline 287 & 401 & 348 & 62 & 449 & 85 & 8 & 480 & 480 & 551 & 386 & 331 & 348 \\
\hline 144 & 377 & 211 & 377 & 326 & 353 & 53 & 479 & 479 & 342 & 445 & 381 & 291 \\
\hline 425 & 172 & 386 & 361 & 301 & 503 & 51 & 422 & 422 & 323 & 414 & 130 & 303 \\
\hline 142 & 573 & 426 & 461 & 370 & 575 & 83 & 456 & 456 & 62 & 384 & 525 & 121 \\
\hline 200 & 44 & 102 & 294 & 382 & 216 & 60 & 459 & 459 & 441 & 378 & 198 & 371 \\
\hline 430 & 43 & 558 & 536 & 380 & 242 & 62 & 452 & 452 & 4 & 72 & 72 & 573 \\
\hline 174 & 446 & 39 & 19 & 360 & 570 & 52 & 22 & 22 & 287 & 490 & 490 & 333 \\
\hline 364 & 388 & 264 & 358 & 375 & 131 & 61 & 471 & 471 & 85 & 7 & 7 & 117 \\
\hline 471 & 5 & 161 & 462 & 571 & 61 & 76 & 300 & 300 & 108 & 49 & 49 & 577 \\
\hline 294 & 46 & 66 & 473 & 385 & 95 & 78 & 7 & 7 & 338 & 31 & 31 & 118 \\
\hline 327 & 225 & 452 & 61 & 361 & 448 & 66 & 23 & 23 & 164 & 528 & 528 & 469 \\
\hline 546 & 306 & 398 & 541 & 293 & 146 & 67 & 520 & 520 & 496 & 507 & 507 & 319 \\
\hline 258 & 246 & 282 & 302 & 542 & 247 & 82 & 291 & 291 & 316 & 132 & 132 & 334 \\
\hline 462 & 6 & 427 & 476 & 327 & 133 & 34 & 485 & 485 & 388 & 330 & 330 & 211 \\
\hline 163 & 480 & 265 & 394 & 3 & 357 & 64 & 534 & 534 & 207 & 71 & 71 & 193 \\
\hline 374 & 457 & 484 & 481 & 352 & 209 & 63 & 353 & 353 & 177 & 58 & 58 & 189 \\
\hline 340 & 240 & 128 & 306 & 322 & 132 & 79 & 357 & 357 & 561 & 44 & 45 & 387 \\
\hline 408 & 175 & 489 & 10 & 520 & 246 & 77 & 348 & 348 & 361 & 45 & 54 & 212 \\
\hline 243 & 235 & 194 & 20 & 461 & 348 & 65 & 470 & 470 & 39 & 54 & 223 & 213 \\
\hline 422 & 134 & 528 & 475 & 290 & 562 & 57 & 468 & 468 & 327 & 223 & 200 & 572 \\
\hline 292 & 353 & 72 & 369 & 347 & 122 & 81 & 539 & 539 & 494 & 200 & 246 & 210 \\
\hline 228 & 157 & 252 & 368 & 1 & 234 & 80 & 325 & 325 & 114 & 246 & 175 & 100 \\
\hline 412 & 223 & 371 & 367 & 443 & 555 & 467 & 378 & 378 & 490 & 175 & 174 & 457 \\
\hline 505 & 93 & 490 & 18 & 324 & 482 & 98 & 463 & 463 & 415 & 174 & 242 & 485 \\
\hline 217 & 425 & 182 & 520 & 316 & 54 & 102 & 317 & 317 & 270 & 242 & 102 & 535 \\
\hline 326 & 200 & 248 & 389 & 450 & 517 & 85 & 527 & 527 & 313 & 189 & 455 & 482 \\
\hline 432 & 174 & 94 & 11 & 313 & 347 & 144 & 14 & 14 & 489 & 22 & 505 & 532 \\
\hline 328 & 471 & 497 & 356 & 321 & 561 & 99 & 576 & 576 & 27 & 211 & 126 & 524 \\
\hline 527 & 340 & 476 & 527 & 318 & 254 & 101 & 524 & 524 & 268 & 193 & 46 & 520 \\
\hline 512 & 228 & 355 & 524 & 524 & 377 & 112 & 538 & 538 & 107 & 524 & 55 & 444 \\
\hline 513 & 323 & 432 & 384 & 527 & 167 & 111 & 312 & 312 & 566 & 390 & 326 & 415 \\
\hline 323 & 71 & 433 & 386 & 536 & 281 & 84 & 328 & 328 & 505 & 140 & 5 & 173 \\
\hline 71 & 195 & 238 & 390 & 310 & 253 & 70 & 499 & 499 & 237 & 389 & 98 & 140 \\
\hline 486 & 555 & 164 & 388 & 304 & 515 & 103 & 478 & 478 & 40 & 527 & 395 & 127 \\
\hline 32 & 563 & 568 & 295 & 147 & 55 & 113 & 320 & 320 & 439 & 139 & 415 & 139 \\
\hline 290 & 506 & 493 & 370 & 462 & 75 & 74 & 292 & 292 & 21 & 245 & 292 & 527 \\
\hline 280 & 7 & 123 & 376 & 482 & 73 & 115 & 305 & 305 & 163 & 43 & 142 & 455 \\
\hline 409 & 86 & 229 & 383 & 358 & 384 & 114 & 496 & 496 & 18 & 131 & 35 & 138 \\
\hline 483 & 242 & 40 & 382 & 400 & 168 & 59 & 498 & 498 & 19 & 172 & 61 & 491 \\
\hline 38 & 487 & 373 & 380 & 395 & 346 & 145 & 311 & 311 & 278 & 491 & 523 & 526 \\
\hline 48 & 492 & 275 & 375 & 473 & 560 & 95 & 303 & 303 & 7 & 222 & 354 & 94 \\
\hline 551 & 325 & 372 & 571 & 401 & 554 & 109 & 121 & 121 & 560 & 517 & 516 & 215 \\
\hline 301 & 320 & 279 & 289 & 9 & 256 & 122 & 323 & 323 & 70 & 532 & 91 & 528 \\
\hline 267 & 39 & 406 & 526 & 52 & 255 & 116 & 5 & 5 & 474 & 450 & 305 & 98 \\
\hline 337 & 521 & 4 & 525 & 457 & 102 & 110 & 2 & 2 & 173 & 482 & 457 & 141 \\
\hline 378 & 507 & 287 & 523 & 575 & 63 & 75 & 4 & 4 & 43 & 127 & 36 & 575 \\
\hline 459 & 490 & 257 & 478 & 485 & 470 & 104 & 16 & 16 & 125 & 457 & 452 & 428 \\
\hline 214 & 141 & 369 & 351 & 372 & 468 & 169 & 6 & 6 & 351 & 36 & 323 & 430 \\
\hline 192 & 431 & 574 & 346 & 371 & 280 & 108 & 354 & 354 & 404 & 150 & 39 & 484 \\
\hline 195 & 132 & 456 & 169 & 526 & 356 & 135 & 15 & 15 & 387 & 243 & 233 & 521 \\
\hline 332 & 244 & 35 & 543 & 573 & 149 & 69 & 501 & 501 & 529 & 76 & 325 & 425 \\
\hline 102 & 72 & 271 & 60 & 480 & 64 & 105 & 355 & 355 & 378 & 128 & 515 & 172 \\
\hline 1 & 58 & 403 & 63 & 518 & 220 & 107 & 120 & 120 & 240 & 458 & 555 & 451 \\
\hline
\end{tabular}




\begin{tabular}{|c|c|c|c|c|c|c|c|c|c|c|c|c|}
\hline 458 & 472 & \begin{tabular}{|l|l}
548 \\
\end{tabular} & 372 & 541 & 472 & 106 & 119 & 119 & 208 & 397 & 504 & 73 \\
\hline 386 & 54 & 446 & 518 & 476 & 539 & 136 & 333 & 333 & 350 & 391 & 488 & 44 \\
\hline 279 & 526 & 531 & 330 & 481 & 201 & 179 & 117 & 117 & 548 & 476 & 92 & 126 \\
\hline 574 & 452 & 431 & 332 & 525 & 24 & 68 & 577 & 577 & 484 & 564 & 93 & 222 \\
\hline 226 & 171 & 340 & 331 & 523 & 439 & 154 & 118 & 118 & 201 & 452 & 195 & 503 \\
\hline 555 & 330 & 442 & 460 & 424 & 474 & 94 & 469 & 469 & 145 & 323 & 451 & 43 \\
\hline 421 & 36 & 526 & 329 & 298 & 177 & 131 & 319 & 319 & 283 & 361 & 320 & 326 \\
\hline 155 & 550 & 366 & 315 & 414 & 71 & 132 & 334 & 334 & 386 & 39 & 492 & 3 \\
\hline 468 & 219 & 562 & 314 & 445 & 556 & 130 & 484 & 484 & 49 & 114 & 576 & 574 \\
\hline 341 & 488 & 425 & 371 & 478 & 345 & 134 & 521 & 521 & 123 & 489 & 487 & 505 \\
\hline 569 & 572 & 166 & 79 & 412 & 325 & 128 & 453 & 453 & 440 & 19 & 171 & 372 \\
\hline 517 & 31 & 505 & 25 & 479 & 134 & 150 & 359 & 359 & 260 & 560 & 234 & 379 \\
\hline 196 & 49 & 37 & 522 & 416 & 431 & 149 & 454 & 454 & 233 & 70 & 480 & 517 \\
\hline 239 & 515 & 382 & 548 & 452 & 573 & 125 & 483 & 483 & 168 & 173 & 62 & 420 \\
\hline 407 & 576 & 215 & 76 & 574 & 139 & 129 & 363 & 363 & 31 & 125 & 85 & 46 \\
\hline 416 & 429 & 399 & 21 & 431 & 712 & 90 & 28 & 28 & 543 & 350 & 40 & 175 \\
\hline 500 & 233 & 240 & 547 & 446 & 442 & 181 & 52 & 52 & 357 & 233 & 474 & 176 \\
\hline 448 & 451 & 151 & 452 & \begin{tabular}{l|l}
356 \\
\end{tabular} & 27 & 133 & 62 & 62 & 364 & 168 & 503 & 525 \\
\hline 563 & 92 & 230 & 477 & 455 & 195 & 180 & 532 & 532 & 435 & 360 & 377 & 474 \\
\hline 406 & 528 & 316 & 531 & 12 & 443 & 151 & 306 & 306 & 360 & 299 & 364 & 174 \\
\hline 506 & 234 & 575 & 529 & 522 & 30 & 146 & 297 & 297 & 530 & 166 & 355 & 480 \\
\hline 7 & 504 & 358 & 448 & 484 & 86 & 89 & 299 & 299 & 400 & 325 & 566 & 131 \\
\hline 410 & 9 & 551 & 296 & 521 & 178 & 182 & 298 & 298 & 275 & 371 & 145 & 391 \\
\hline 424 & 495 & 498 & 308 & 451 & 461 & 183 & 519 & 519 & 528 & 426 & 275 & 472 \\
\hline 418 & 317 & 389 & 453 & 53 & 189 & 153 & 79 & 79 & 299 & 96 & 437 & 40 \\
\hline 542 & 45 & 428 & 482 & 51 & 340 & 152 & 308 & 308 & 292 & 103 & 454 & 506 \\
\hline 557 & 484 & 247 & 362 & 475 & 218 & 215 & 260 & 260 & 136 & 509 & 143 & 200 \\
\hline 14 & 176 & 43 & 449 & 62 & 66 & 73 & 80 & 80 & 60 & 115 & 129 & 523 \\
\hline 360 & 461 & 474 & 309 & 460 & 279 & 222 & 98 & 98 & 8 & 423 & 522 & 223 \\
\hline 544 & 76 & 454 & 480 & 548 & 18 & 175 & 252 & 252 & 288 & 420 & 120 & 533 \\
\hline 359 & 164 & 125 & 24 & 572 & 480 & 174 & 233 & 233 & 550 & 485 & 226 & 92 \\
\hline 81 & 358 & 327 & 83 & 434 & 31 & 200 & 81 & 81 & 507 & 541 & 261 & 322 \\
\hline 86 & 357 & 295 & 442 & 397 & 203 & 223 & 82 & 82 & 248 & 214 & 568 & 58 \\
\hline 145 & 156 & 19 & 479 & 294 & 173 & 58 & 8 & 8 & 166 & 170 & 392 & 245 \\
\hline 194 & 497 & 145 & 441 & 547 & 230 & 71 & 9 & 9 & 314 & 570 & 274 & 25 \\
\hline 8 & 262 & 98 & 440 & 391 & 309 & 228 & 20 & 20 & 142 & 515 & 324 & 102 \\
\hline 178 & 518 & 283 & 413 & 19 & 269 & 72 & 25 & 25 & 132 & 555 & 453 & 392 \\
\hline 242 & 137 & 573 & 439 & 453 & 378 & 195 & 535 & 535 & 325 & 373 & 569 & 490 \\
\hline 545 & 423 & 323 & 396 & 435 & 516 & 86 & 19 & 19 & 126 & 144 & 422 & 71 \\
\hline 206 & 96 & 253 & 407 & 408 & 424 & 91 & 46 & 46 & 46 & 90 & 119 & 452 \\
\hline 376 & 97 & 155 & 436 & 392 & 32 & 214 & 29 & 29 & 66 & 311 & 379 & 225 \\
\hline 351 & 556 & 131 & 404 & 403 & 65 & 168 & 53 & 53 & 417 & 111 & 321 & 55 \\
\hline 516 & 198 & 153 & 417 & 330 & 238 & 166 & 169 & 169 & 448 & 504 & 99 & 39 \\
\hline 382 & 481 & 306 & 438 & 529 & 108 & 88 & 548 & 548 & 461 & 101 & 501 & 518 \\
\hline 158 & 20 & 485 & 405 & 437 & 563 & 167 & 114 & 114 & 371 & 488 & 150 & 507 \\
\hline 335 & 509 & 550 & 406 & 531 & 574 & 87 & 549 & 549 & 140 & 540 & 243 & 45 \\
\hline 182 & 144 & 470 & 485 & 351 & 128 & 123 & 232 & 232 & 426 & 84 & 76 & 254 \\
\hline 479 & 142 & 503 & 359 & 297 & 51 & 165 & 550 & 550 & 436 & 262 & 128 & 132 \\
\hline 108 & 430 & 341 & 385 & 332 & 349 & 881 & 39 & 39 & 169 & 10 & 458 & 242 \\
\hline 571 & 327 & 108 & 484 & 331 & 103 & 227 & 228 & 228 & 35 & 92 & 397 & 293 \\
\hline 487 & 163 & 451 & 521 & 419 & 138 & 173 & 193 & 193 & 96 & 93 & 391 & 228 \\
\hline 454 & 243 & 292 & 450 & 418 & 463 & 127 & 44 & 44 & 103 & 356 & 476 & 504 \\
\hline 177 & 292 & 233 & 114 & 346 & 174 & 172 & 144 & 144 & 437 & 322 & 564 & 72 \\
\hline 476 & 551 & 368 & 400 & 456 & 21 & 126 & 113 & 113 & 509 & 553 & 361 & 142 \\
\hline 492 & 301 & 168 & 401 & 289 & 49 & 176 & 76 & 76 & 182 & 301 & 114 & 488 \\
\hline 336 & 214 & 7 & 67 & 543 & 204 & 92 & 112 & 112 & 23 & 499 & 489 & 244 \\
\hline 450 & 332 & 107 & 26 & 477 & 336 & 171 & 164 & 164 & 500 & 195 & 19 & 462 \\
\hline 138 & 458 & 380 & 64 & 302 & 56 & 198 & 222 & 222 & 470 & 481 & 560 & 171 \\
\hline 325 & 279 & 444 & 36 & 61 & 43 & 93 & 77 & 77 & 55 & 421 & 70 & 234 \\
\hline 245 & 421 & 260 & 35 & 329 & 171 & 170 & 78 & 78 & 405 & 451 & 125 & 492 \\
\hline 260 & 155 & 177 & 395 & 362 & 140 & 124 & 290 & 290 & 410 & 349 & 350 & 198 \\
\hline 352 & 341 & 525 & 456 & 381 & 72 & 216 & 58 & 58 & 216 & 149 & 168 & 487 \\
\hline 126 & 500 & 288 & 307 & 10 & 158 & 177 & 223 & 223 & 503 & 533 & 360 & 93 \\
\hline 62 & 542 & 523 & 544 & 315 & 326 & 202 & 94 & 94 & 382 & 320 & 299 & 49 \\
\hline 274 & 360 & 378 & 349 & 314 & 93 & 197 & 59 & 59 & 77 & 537 & 166 & 473 \\
\hline 89 & 516 & 488 & 350 & 503 & 390 & 196 & 97 & 97 & 115 & 300 & 371 & 360 \\
\hline 266 & 479 & 49 & 530 & 20 & 96 & 199 & 289 & 289 & 253 & 479 & 426 & 233 \\
\hline 263 & 177 & 342 & 260 & 499 & 283 & 148 & 235 & 235 & 427 & 492 & 96 & 324 \\
\hline 103 & 476 & 76 & 80 & 18 & 317 & 201 & 137 & 137 & 167 & 113 & 103 & 1 \\
\hline 282 & 103 & 570 & 451 & 530 & 26 & 217 & 250 & 250 & 403 & 539 & 509 & 411 \\
\hline 415 & 282 & 367 & 30 & 515 & 262 & 236 & 288 & 288 & 238 & 576 & 115 & 499 \\
\hline 320 & 415 & 499 & 32 & 25 & 414 & 157 & 211 & 211 & 153 & 312 & 423 & 85 \\
\hline 107 & 331 & 235 & 447 & 444 & 196 & 137 & 249 & 249 & 29 & 78 & 420 & 429 \\
\hline 241 & 170 & 385 & 391 & 306 & 42 & 97 & 248 & 248 & 87 & 487 & 541 & 246 \\
\hline 68 & 124 & 522 & 392 & 11 & 60 & 156 & 47 & 47 & 330 & 511 & 214 & 36 \\
\hline 39 & 168 & 557 & 575 & 399 & 107 & 96 & 157 & 157 & 328 & 171 & 170 & 361 \\
\hline 460 & 395 & 201 & 77 & 516 & 491 & 155 & 287 & 287 & 339 & 124 & 570 & 195 \\
\hline 521 & 269 & 22 & 288 & 359 & 148 & 187 & 100 & 100 & 131 & 370 & 144 & 54 \\
\hline 321 & 95 & 338 & 381 & 454 & 125 & 186 & 109 & 109 & 423 & 97 & 90 & 31 \\
\hline
\end{tabular}




\begin{tabular}{|c|c|c|c|c|c|c|c|c|c|c|c|c|}
\hline 562 & 130 & 328 & 336 & 458 & 504 & 206 & 518 & 518 & 296 & 542 & 311 & 500 \\
\hline 561 & 90 & 103 & 50 & 169 & 183 & 205 & 225 & 225 & 83 & 554 & 111 & 62 \\
\hline 308 & 61 & 407 & 113 & 60 & 104 & 207 & 111 & 111 & 247 & 234 & 101 & 28 \\
\hline 445 & 311 & 401 & 34 & 349 & 150 & 184 & 200 & 200 & 420 & 112 & 540 & 515 \\
\hline 507 & 539 & 417 & 345 & 295 & 425 & 185 & 36 & 36 & 558 & 137 & 84 & 130 \\
\hline 208 & 387 & 140 & 515 & 425 & 527 & 209 & 282 & 282 & 485 & 52 & 262 & 235 \\
\hline 331 & 78 & 507 & 516 & 486 & 452 & 231 & 219 & 219 & 454 & 480 & 10 & 259 \\
\hline 522 & 216 & 71 & 39 & 350 & 50 & 221 & 110 & 110 & 541 & 536 & 356 & 330 \\
\hline 170 & 420 & 509 & 443 & 483 & 127 & 220 & 74 & 74 & 143 & 156 & 322 & 86 \\
\hline 124 & 19 & 461 & 66 & 79 & 358 & 178 & 175 & 175 & 556 & 293 & 553 & 27 \\
\hline 168 & 113 & 375 & 454 & 76 & 542 & 712 & 215 & 215 & 285 & 518 & 301 & 357 \\
\hline 520 & 112 & 142 & 457 & 63 & 181 & 203 & 533 & 533 & 329 & 100 & 499 & 522 \\
\hline 490 & 2 & 8 & 57 & 500 & 229 & 251 & 188 & 188 & 214 & 102 & 481 & 164 \\
\hline 33 & 232 & 62 & 549 & 549 & 126 & 193 & 35 & 35 & 303 & 455 & 421 & 327 \\
\hline 141 & 3 & 492 & 33 & 430 & 341 & 189 & 259 & 259 & 456 & 505 & 349 & 398 \\
\hline 88 & 434 & 376 & 31 & 544 & 67 & 212 & 208 & 208 & 170 & 126 & 149 & 91 \\
\hline 395 & 300 & 258 & 65 & 398 & 449 & 213 & 85 & 85 & 372 & 46 & 533 & 29 \\
\hline 269 & 66 & 463 & 252 & 428 & 407 & 210 & 503 & 503 & 16 & 55 & 537 & 13 \\
\hline 289 & 297 & 132 & 55 & 420 & 14 & 100 & 216 & 216 & 235 & 326 & 300 & 144 \\
\hline 143 & 111 & 278 & 455 & 46 & 83 & 140 & 242 & 242 & 345 & 5 & 479 & 226 \\
\hline 95 & 525 & 351 & 546 & 36 & 450 & 139 & 570 & 570 & 59 & 443 & 113 & 461 \\
\hline 130 & 536 & 364 & 112 & 345 & 156 & 138 & 131 & 131 & 37 & 215 & 539 & 540 \\
\hline 436 & 523 & 471 & 115 & 21 & 170 & 141 & 61 & 61 & 81 & 73 & 312 & 555 \\
\hline 34 & 350 & 350 & 499 & 415 & 495 & 142 & 95 & 95 & 411 & 366 & 78 & 495 \\
\hline 146 & 181 & 126 & 37 & 44 & 87 & 164 & 146 & 146 & 61 & 447 & 511 & 240 \\
\hline 90 & 426 & 87 & 483 & 517 & 576 & 226 & 247 & 247 & 257 & 98 & 124 & 536 \\
\hline 61 & 554 & 473 & 363 & 546 & 258 & 158 & 133 & 133 & 277 & 62 & 370 & 35 \\
\hline 559 & 128 & 173 & 81 & 35 & 124 & 143 & 209 & 209 & 445 & 85 & 97 & 537 \\
\hline 307 & 101 & 58 & 44 & 24 & 115 & 194 & 132 & 132 & 570 & 40 & 542 & 458 \\
\hline 28 & 428 & 333 & 431 & 411 & 524 & 190 & 246 & 246 & 366 & 474 & 554 & 433 \\
\hline 311 & 499 & 381 & 545 & 288 & 106 & 163 & 562 & 562 & 531 & 503 & 112 & 542 \\
\hline 539 & 52 & 149 & 424 & 296 & 105 & 162 & 122 & 122 & 271 & 377 & 137 & 516 \\
\hline 251 & 115 & 477 & 503 & 309 & 293 & 159 & 234 & 234 & 515 & 535 & 52 & 99 \\
\hline 431 & 305 & 32 & 414 & 498 & 538 & 160 & 555 & 555 & 555 & 94 & 536 & 299 \\
\hline 132 & 397 & 555 & 412 & 114 & 389 & 191 & 54 & 54 & 373 & 519 & 156 & 479 \\
\hline 57 & 125 & 325 & 416 & 488 & 488 & 192 & 517 & 517 & 187 & 495 & 293 & 550 \\
\hline 387 & 322 & 31 & 233 & 308 & 417 & 161 & 347 & 347 & 463 & 317 & 518 & 158 \\
\hline 78 & 84 & 167 & 397 & 504 & 155 & 246 & 561 & 561 & 71 & 563 & 495 & 243 \\
\hline 47 & 42 & 556 & 434 & 39 & 163 & 219 & 254 & 254 & 104 & 429 & 317 & 489 \\
\hline 213 & 511 & 361 & 435 & 83 & 404 & 232 & 167 & 167 & 557 & 425 & 563 & 134 \\
\hline 216 & 35 & 206 & 408 & 497 & 361 & 248 & 281 & 281 & 471 & 550 & 429 & 209 \\
\hline 538 & 356 & 216 & 403 & 55 & 339 & 247 & 253 & 253 & 105 & 471 & 425 & 301 \\
\hline 183 & 209 & 504 & 437 & 215 & 70 & 188 & 515 & 515 & 380 & 228 & 550 & 332 \\
\hline 237 & 564 & 370 & 486 & 433 & 135 & 208 & 55 & 55 & 144 & 157 & 471 & 331 \\
\hline 420 & 361 & 44 & 419 & 228 & 159 & 230 & 75 & 75 & 58 & 431 & 228 & 101 \\
\hline 19 & 91 & 374 & 418 & 487 & 197 & 218 & 73 & 73 & 90 & 9 & 157 & 8 \\
\hline 319 & 67 & 357 & 56 & 31 & 284 & 250 & 168 & 168 & 151 & 472 & 431 & 9 \\
\hline 113 & 537 & 360 & 399 & 43 & 278 & 238 & 346 & 346 & 109 & 353 & 9 & 128 \\
\hline 193 & 299 & 466 & 573 & 233 & 564 & 249 & 560 & 560 & 228 & 259 & 472 & 42 \\
\hline 529 & 478 & 478 & 78 & 409 & 268 & 204 & 554 & 554 & 416 & 575 & 353 & 409 \\
\hline 112 & 109 & 329 & 574 & 67 & 312 & 211 & 256 & 256 & 32 & 364 & 259 & 170 \\
\hline 188 & 150 & 521 & 54 & 393 & 263 & 245 & 255 & 255 & 311 & 254 & 575 & 321 \\
\hline 244 & 349 & 549 & 49 & 222 & 360 & 225 & \begin{tabular}{|l|}
102 \\
\end{tabular} & \begin{tabular}{l|l}
102 \\
\end{tabular} & 562 & 25 & 254 & 511 \\
\hline 72 & 541 & 468 & 467 & 511 & 328 & 242 & 63 & 63 & 116 & 17 & 25 & 214 \\
\hline 227 & 560 & 313 & 445 & 64 & 445 & 244 & 280 & 280 & 493 & 219 & 219 & 157 \\
\hline 324 & 59 & 143 & 40 & 80 & 245 & 240 & 356 & 356 & 69 & 135 & 135 & 399 \\
\hline 255 & 553 & 541 & 446 & 30 & 499 & 243 & 149 & 149 & 478 & 64 & 64 & 563 \\
\hline 222 & 64 & 85 & 572 & 98 & 180 & 224 & 64 & 64 & 881 & 155 & 155 & 143 \\
\hline 427 & 149 & 546 & 85 & 550 & 497 & 257 & 220 & 220 & 111 & 225 & 225 & 453 \\
\hline 30 & 531 & 483 & 46 & 58 & 92 & 239 & 472 & 472 & 121 & 95 & 95 & 509 \\
\hline 129 & 293 & 481 & 111 & 49 & 455 & 229 & 201 & 201 & 284 & 159 & 159 & 481 \\
\hline 2 & 489 & 415 & 341 & 363 & 237 & 237 & 24 & 24 & 369 & 544 & 544 & 426 \\
\hline 390 & 370 & 538 & 228 & 26 & 386 & 241 & 474 & 474 & 333 & 209 & 209 & 52 \\
\hline 474 & 570 & 41 & 116 & 200 & 221 & 254 & 177 & 177 & 157 & 20 & 20 & 61 \\
\hline 85 & 391 & 449 & 82 & 545 & 478 & 234 & 71 & 71 & 504 & 180 & 180 & 112 \\
\hline 58 & 166 & 18 & 393 & 336 & 500 & 235 & 556 & 556 & 101 & 434 & 434 & 486 \\
\hline 232 & 312 & 377 & 287 & 32 & 240 & 259 & 345 & 345 & 538 & 42 & 42 & 150 \\
\hline 4 & 371 & 423 & 340 & 491 & 151 & 262 & 134 & 134 & 526 & 279 & 279 & 476 \\
\hline 3 & 540 & 303 & 517 & 40 & 457 & 269 & 139 & 139 & 406 & 306 & 306 & 20 \\
\hline 434 & 381 & 104 & 43 & 341 & 543 & 253 & 712 & 712 & 294 & 340 & 340 & 149 \\
\hline 472 & 533 & 392 & 444 & 54 & 116 & 256 & 27 & 27 & 498 & 217 & 217 & 541 \\
\hline 577 & 70 & 21 & 514 & 402 & 13 & 255 & 195 & 195 & 508 & 190 & 190 & 194 \\
\hline 249 & 114 & 284 & 45 & 34 & 187 & 275 & 30 & 30 & 375 & 430 & 430 & 352 \\
\hline 300 & 248 & 144 & 234 & 50 & 396 & 274 & 86 & 86 & 205 & 358 & 358 & 340 \\
\hline 383 & 10 & 188 & 458 & 514 & 40 & 261 & 178 & 178 & 477 & 348 & 348 & 306 \\
\hline 54 & 354 & 27 & 84 & 77 & 881 & 258 & 461 & 461 & 298 & 160 & 160 & 111 \\
\hline 82 & 231 & 122 & 550 & 113 & 451 & 263 & 189 & 189 & 44 & 220 & 220 & 553 \\
\hline 565 & 186 & 207 & 222 & 223 & 557 & 270 & 340 & 340 & 229 & 134 & 134 & 219 \\
\hline 51 & 133 & 59 & 122 & 260 & 412 & 268 & 218 & 218 & 497 & 252 & 252 & 423 \\
\hline
\end{tabular}




\begin{tabular}{|c|c|c|c|c|c|c|c|c|c|c|c|c|}
\hline 473 & 12 & 540 & 498 & 234 & 383 & 272 & 66 & 66 & 376 & 63 & 63 & 76 \\
\hline 534 & 187 & 129 & 97 & 45 & 436 & 271 & 279 & 279 & 377 & 513 & 513 & 84 \\
\hline 80 & 548 & 281 & 42 & 340 & 320 & 264 & 18 & 18 & 110 & 86 & 86 & 316 \\
\hline 66 & 261 & 46 & 167 & 495 & 551 & 267 & 31 & 31 & 332 & 269 & 269 & 570 \\
\hline 79 & 568 & 105 & 98 & 252 & 294 & 265 & 203 & 203 & 488 & 282 & 282 & 190 \\
\hline 297 & 148 & 508 & 232 & 37 & 166 & 266 & 173 & 173 & 362 & 545 & 545 & 19 \\
\hline 212 & 411 & 510 & 47 & 66 & 420 & 273 & 230 & 230 & 549 & 295 & 295 & 554 \\
\hline 111 & 203 & 881 & 96 & 307 & 408 & 290 & 309 & 309 & 106 & 559 & 559 & 163 \\
\hline 402 & 99 & 506 & 398 & 429 & 231 & 282 & 269 & 269 & 540 & 250 & 250 & 224 \\
\hline 347 & 291 & 339 & 110 & 232 & 202 & 279 & 516 & 516 & 473 & 165 & 165 & 70 \\
\hline 525 & 207 & 210 & 488 & 506 & 376 & 281 & 32 & 32 & 280 & 197 & 197 & 137 \\
\hline 536 & 37 & 272 & 215 & 42 & 487 & 287 & 65 & 65 & 565 & 218 & 218 & 168 \\
\hline 453 & 288 & 170 & 487 & 175 & 292 & 280 & 238 & 238 & 468 & 249 & 249 & 478 \\
\hline 523 & 514 & 56 & 504 & 100 & 305 & 283 & 108 & 108 & 510 & 512 & 512 & 103 \\
\hline 197 & 277 & 60 & 430 & 73 & 428 & 278 & 563 & 563 & 281 & 194 & 194 & 356 \\
\hline 127 & 309 & 491 & 425 & 112 & 352 & 289 & 128 & 128 & 82 & 256 & 256 & 166 \\
\hline 55 & 256 & 130 & 428 & 47 & 84 & 288 & 51 & 51 & 575 & 264 & 264 & 232 \\
\hline 56 & 275 & 221 & 41 & 81 & 547 & 284 & 349 & 349 & 30 & 181 & 181 & 421 \\
\hline 350 & 456 & 296 & 58 & 57 & 186 & 277 & 103 & 103 & 202 & 206 & 206 & 125 \\
\hline 15 & 265 & 479 & 511 & 115 & 441 & 285 & 138 & 138 & 84 & 65 & 65 & 313 \\
\hline 13 & 205 & 121 & 95 & 85 & 440 & 335 & 174 & 174 & 309 & 265 & 265 & 113 \\
\hline 229 & 83 & 88 & 420 & 33 & 257 & 276 & 21 & 21 & 262 & 341 & 341 & 97 \\
\hline 29 & 287 & 314 & 415 & 193 & 537 & 286 & 49 & 49 & 331 & 551 & 551 & 349 \\
\hline 342 & 294 & 244 & 411 & 505 & 434 & 300 & 204 & 204 & 10 & 342 & 342 & 10 \\
\hline 201 & 546 & 172 & 433 & 71 & 460 & 233 & 336 & 336 & 374 & 4 & 4 & 262 \\
\hline 526 & 258 & 393 & 409 & 82 & 272 & 299 & 56 & 56 & 424 & 287 & 287 & 156 \\
\hline 40 & 462 & 413 & 402 & 65 & 479 & 301 & 43 & 43 & 230 & 108 & 108 & 402 \\
\hline 401 & 408 & 90 & 200 & 426 & 514 & 306 & 171 & 171 & 297 & 338 & 338 & 129 \\
\hline 181 & 422 & 228 & 500 & 423 & 400 & 297 & 140 & 140 & 92 & 164 & 164 & 88 \\
\hline 268 & 217 & 106 & 137 & 287 & 162 & 336 & 72 & 72 & 172 & 496 & 496 & 548 \\
\hline 558 & 432 & 542 & 223 & 94 & 496 & 252 & 158 & 158 & 80 & 316 & 316 & 269 \\
\hline 398 & 328 & 120 & 168 & 507 & 34 & 298 & 326 & 326 & 466 & 207 & 207 & 358 \\
\hline 433 & 512 & 262 & 144 & 127 & 481 & 309 & 93 & 93 & 398 & 177 & 177 & 96 \\
\hline 426 & 513 & 280 & 497 & 459 & 498 & 294 & 96 & 96 & 93 & 561 & 561 & 341 \\
\hline 385 & 486 & 285 & 70 & 195 & 430 & 308 & 283 & 283 & 14 & 327 & 327 & 547 \\
\hline 452 & 32 & 330 & 75 & 144 & 486 & 296 & 26 & 26 & 258 & 494 & 494 & 551 \\
\hline 442 & 290 & 569 & 491 & 59 & 172 & 260 & 262 & 262 & 356 & 270 & 270 & 78 \\
\hline 210 & 280 & 267 & 109 & 78 & 528 & 295 & 196 & 196 & 322 & 313 & 313 & 350 \\
\hline 372 & 409 & 183 & 342 & 421 & 89 & 307 & 42 & 42 & 553 & 27 & 27 & 162 \\
\hline 554 & 267 & 539 & 506 & 174 & 57 & 302 & 60 & 60 & 506 & 268 & 268 & 454 \\
\hline 171 & 1 & 487 & 513 & 167 & 12 & 317 & 107 & 107 & 407 & 107 & 107 & 74 \\
\hline 494 & 574 & 553 & 59 & 496 & 267 & 312 & 491 & 491 & 100 & 237 & 237 & 531 \\
\hline 530 & 226 & 520 & 102 & 72 & 311 & 311 & 148 & 148 & 389 & 21 & 21 & 115 \\
\hline 377 & 569 & 193 & 282 & 173 & 303 & 316 & 125 & 125 & 301 & 163 & 163 & 124 \\
\hline 128 & 196 & 114 & 507 & 211 & 121 & 313 & 504 & 504 & 41 & 18 & 18 & 497 \\
\hline 247 & 239 & 83 & 339 & 489 & 301 & 318 & 183 & 183 & 499 & 278 & 278 & 253 \\
\hline 74 & 410 & 236 & 344 & 102 & 391 & 310 & 104 & 104 & 195 & 351 & 351 & 37 \\
\hline 881 & 557 & 345 & 250 & 111 & 403 & 315 & 150 & 150 & 481 & 387 & 387 & 90 \\
\hline 172 & 544 & 67 & 216 & 56 & 362 & 314 & 50 & 50 & 45 & 529 & 529 & 114 \\
\hline 50 & 81 & 100 & 253 & 427 & 458 & 326 & 127 & 127 & 421 & 240 & 240 & 560 \\
\hline 101 & 145 & 92 & 100 & 168 & 45 & 330 & 542 & 542 & 451 & 208 & 208 & 347 \\
\hline 348 & 194 & 55 & 495 & 86 & 507 & 327 & 181 & 181 & 523 & 548 & 548 & 290 \\
\hline 75 & 8 & 202 & 570 & 93 & 509 & 332 & 229 & 229 & 399 & 201 & 201 & 530 \\
\hline 257 & 178 & 533 & 555 & 432 & 214 & 331 & 126 & 126 & 221 & 283 & 283 & 564 \\
\hline 330 & 545 & 70 & 48 & 490 & 101 & 329 & 341 & 341 & 129 & 123 & 123 & 297 \\
\hline 36 & 206 & 424 & 459 & 216 & 342 & 364 & 67 & 67 & 349 & 260 & 260 & 231 \\
\hline 343 & 351 & 511 & 157 & 509 & 338 & 365 & 83 & 83 & 381 & 543 & 543 & 216 \\
\hline 428 & 158 & 30 & 551 & 235 & 69 & 360 & 156 & 156 & 210 & 357 & 357 & 159 \\
\hline 493 & 182 & 534 & 164 & 555 & 136 & 361 & 170 & 170 & 130 & 435 & 435 & 256 \\
\hline 470 & 108 & 472 & 195 & 492 & 160 & 357 & 495 & 495 & 352 & 530 & 530 & 255 \\
\hline 499 & 454 & 515 & 146 & 189 & 565 & 352 & 87 & 87 & 522 & 136 & 136 & 556 \\
\hline 524 & 336 & 75 & 73 & 164 & 285 & 340 & 258 & 258 & 442 & 60 & 60 & 59 \\
\hline 52 & 260 & 93 & 71 & 138 & 217 & 356 & 124 & 124 & 286 & 8 & 8 & 41 \\
\hline 115 & 352 & 203 & 101 & 513 & 277 & 349 & 115 & 115 & 149 & 288 & 288 & 529 \\
\hline 369 & 274 & 712 & 175 & 410 & 323 & 358 & 106 & 106 & 120 & 248 & 248 & 66 \\
\hline 305 & 89 & 294 & 99 & 570 & 316 & 341 & 105 & 105 & 432 & 314 & 314 & 67 \\
\hline 295 & 266 & 109 & 72 & 41 & 327 & 350 & 293 & 293 & 533 & 66 & 66 & 456 \\
\hline 373 & 263 & 322 & 166 & 116 & 313 & 351 & 488 & 488 & 11 & 461 & 461 & 82 \\
\hline 162 & 107 & 410 & 214 & 74 & 264 & 345 & 155 & 155 & 412 & 169 & 169 & 47 \\
\hline 550 & 460 & 537 & 155 & 92 & 5 & 339 & 163 & 163 & 56 & 182 & 182 & 145 \\
\hline 27 & 321 & 61 & 156 & 122 & 91 & 362 & 339 & 339 & 534 & 500 & 500 & 177 \\
\hline 397 & 562 & 416 & 489 & 214 & 295 & 346 & 70 & 70 & 326 & 410 & 410 & 95 \\
\hline 508 & 561 & 57 & 505 & 91 & 182 & 342 & 135 & 135 & 203 & 216 & 216 & 318 \\
\hline 105 & 308 & 354 & 429 & 95 & 387 & 338 & 159 & 159 & 57 & 77 & 77 & 248 \\
\hline 125 & 208 & 23 & 509 & 84 & 544 & 344 & 197 & 197 & 186 & 253 & 253 & 181 \\
\hline 219 & 522 & 171 & 427 & 126 & 492 & 359 & 284 & 284 & 525 & 427 & 427 & 109 \\
\hline 379 & 88 & 77 & 426 & 139 & 397 & 363 & 278 & 278 & 433 & 167 & 167 & 282 \\
\hline 488 & 289 & 81 & 423 & 75 & 444 & 343 & 564 & 564 & 574 & 403 & 403 & 329 \\
\hline 573 & 143 & 356 & 512 & 551 & 243 & 337 & 268 & 268 & 308 & 238 & 238 & 275 \\
\hline
\end{tabular}




\begin{tabular}{|c|c|c|c|c|c|c|c|c|c|c|c|c|}
\hline 322 & 34 & 480 & 432 & 140 & 511 & 394 & 263 & 263 & 191 & 153 & 153 & 274 \\
\hline 712 & 146 & 332 & 421 & 253 & 165 & 396 & 245 & 245 & 133 & 29 & 29 & 261 \\
\hline 368 & 559 & 101 & 135 & 131 & 476 & 395 & 180 & 180 & 320 & 87 & 87 & 32 \\
\hline 22 & 28 & 214 & 188 & 254 & 190 & 373 & 92 & 92 & 368 & 328 & 328 & 34 \\
\hline 285 & 57 & 255 & 410 & 97 & 296 & 389 & 237 & 237 & 122 & 339 & 339 & 122 \\
\hline 496 & 47 & 299 & 133 & 132 & 224 & 390 & 221 & 221 & 385 & 296 & 296 & 427 \\
\hline 510 & 538 & 195 & 74 & 250 & 2 & 388 & 240 & 240 & 67 & 83 & 83 & 167 \\
\hline 84 & 183 & 460 & 138 & 166 & 536 & 384 & 151 & 151 & 367 & 247 & 247 & 64 \\
\hline 264 & 237 & 274 & 490 & 512 & 456 & 370 & 543 & 543 & 537 & 558 & 558 & 63 \\
\hline 253 & 529 & 16 & 193 & 210 & 90 & 386 & 116 & 116 & 431 & 556 & 556 & 258 \\
\hline 441 & 188 & 420 & 492 & 212 & 10 & 397 & 13 & 13 & 300 & 285 & 285 & 310 \\
\hline 77 & 324 & 411 & 108 & 213 & 206 & 383 & 187 & 187 & 6 & 329 & 329 & 155 \\
\hline 160 & 255 & 246 & 235 & 467 & 464 & 374 & 40 & 40 & 491 & 303 & 303 & 336 \\
\hline 199 & 427 & 349 & 249 & 99 & 37 & 376 & 881 & 881 & 479 & 456 & 456 & 257 \\
\hline 375 & 30 & 82 & 496 & 101 & 540 & 382 & 557 & 557 & 492 & 372 & 372 & 202 \\
\hline 44 & 129 & 277 & 201 & 225 & 558 & 380 & 551 & 551 & 113 & 235 & 235 & 247 \\
\hline 463 & 4 & 352 & 103 & 342 & 505 & 375 & 294 & 294 & 231 & 345 & 345 & 50 \\
\hline 314 & 249 & 331 & 69 & 110 & 423 & 571 & 166 & 166 & 2 & 59 & 59 & 279 \\
\hline 42 & 82 & 402 & 132 & 282 & 411 & 385 & 231 & 231 & 401 & 37 & 37 & 496 \\
\hline 572 & 565 & 169 & 93 & 96 & 385 & 449 & 202 & 202 & 539 & 81 & 81 & 498 \\
\hline 23 & 51 & 532 & 208 & 141 & 421 & 400 & 487 & 487 & 521 & 411 & 411 & 432 \\
\hline 31 & 473 & 187 & 139 & 88 & 212 & 401 & 352 & 352 & 310 & 257 & 257 & 239 \\
\hline 69 & 534 & 326 & 140 & 158 & 213 & 381 & 84 & 84 & 446 & 277 & 277 & 569 \\
\hline 152 & 80 & 320 & 141 & 494 & 438 & 371 & 547 & 547 & 193 & 531 & 531 & 133 \\
\hline 404 & 79 & 266 & 254 & 339 & 531 & 387 & 186 & 186 & 148 & 271 & 271 & 79 \\
\hline 49 & 347 & 204 & 343 & 109 & 416 & 372 & 257 & 257 & 9 & 187 & 187 & 493 \\
\hline 333 & 453 & 308 & 145 & 201 & 409 & 391 & 537 & 537 & 3 & 104 & 104 & 549 \\
\hline 511 & 197 & 29 & 174 & 137 & 11 & 392 & 460 & 460 & 428 & 557 & 557 & 87 \\
\hline 405 & 56 & 96 & 86 & 172 & 123 & 398 & 272 & 272 & 5 & 105 & 105 & 483 \\
\hline 498 & 13 & 565 & 196 & 209 & 351 & 399 & 514 & 514 & 88 & 151 & 151 & 30 \\
\hline 35 & 229 & 552 & 281 & 177 & 513 & 393 & 162 & 162 & 198 & 109 & 109 & 252 \\
\hline 110 & 29 & 286 & 91 & 70 & 546 & 431 & 34 & 34 & 576 & 32 & 32 & 460 \\
\hline 515 & 342 & 198 & 283 & 130 & 506 & 414 & 481 & 481 & 188 & 562 & 562 & 221 \\
\hline 501 & 201 & 163 & 158 & 196 & 244 & 416 & 486 & 486 & 75 & 116 & 116 & 477 \\
\hline 278 & 268 & 178 & 127 & 202 & 205 & 424 & 172 & 172 & 289 & 493 & 493 & 197 \\
\hline 356 & 558 & 110 & 508 & 249 & 489 & 412 & 528 & 528 & 89 & 69 & 69 & 298 \\
\hline 355 & 398 & 141 & 338 & 344 & 194 & 415 & 89 & 89 & 161 & 478 & 478 & 494 \\
\hline 209 & 433 & 261 & 194 & 157 & 382 & 428 & 57 & 57 & 312 & 881 & 881 & 196 \\
\hline 218 & 372 & 111 & 173 & 48 & 380 & 430 & 12 & 12 & 354 & 121 & 121 & 123 \\
\hline 380 & 494 & 115 & 494 & 128 & 433 & 425 & 267 & 267 & 79 & 284 & 284 & 514 \\
\hline 238 & 530 & 133 & 165 & 133 & 541 & 420 & 301 & 301 & 472 & 333 & 333 & 199 \\
\hline 18 & 247 & 89 & 88 & 493 & 271 & 411 & 45 & 45 & 337 & 538 & 538 & 422 \\
\hline 60 & 74 & 148 & 219 & 134 & 494 & 429 & 507 & 507 & 78 & 294 & 294 & 77 \\
\hline 363 & 881 & 138 & 142 & 248 & 395 & 409 & 509 & 509 & 53 & 498 & 498 & 116 \\
\hline 393 & 50 & 347 & 554 & 170 & 4 & 426 & 214 & 214 & 712 & 508 & 508 & 180 \\
\hline 564 & 348 & 412 & 562 & 165 & 399 & 423 & 101 & 101 & 573 & 205 & 205 & 151 \\
\hline 345 & 75 & 157 & 143 & 103 & 210 & 421 & 342 & 342 & 413 & 477 & 477 & 110 \\
\hline 346 & 257 & 263 & 553 & 146 & 462 & 427 & 338 & 338 & 487 & 298 & 298 & 304 \\
\hline 365 & 493 & 289 & 94 & 554 & 530 & 422 & 69 & 69 & 244 & 229 & 229 & 147 \\
\hline 179 & 295 & 502 & 187 & 242 & 545 & 410 & 136 & 136 & 226 & 497 & 497 & 188 \\
\hline 389 & 162 & 80 & 552 & 194 & 512 & 413 & 160 & 160 & 184 & 110 & 110 & 309 \\
\hline 123 & 27 & 45 & 87 & 219 & 446 & 442 & 565 & 565 & 272 & 362 & 362 & 294 \\
\hline 344 & 105 & 297 & 211 & 422 & 41 & 369 & 285 & 285 & 212 & 549 & 549 & 18 \\
\hline 135 & 379 & 24 & 131 & 87 & 529 & 368 & 217 & 217 & 222 & 106 & 106 & 148 \\
\hline 180 & 712 & 309 & 179 & 176 & 477 & 367 & 277 & 277 & 511 & 473 & 473 & 187 \\
\hline 444 & 285 & 337 & 493 & 123 & 388 & 441 & 316 & 316 & 483 & 280 & 280 & 559 \\
\hline 304 & 496 & 475 & 162 & 142 & 16 & 440 & 327 & 327 & 276 & 565 & 565 & 208 \\
\hline 122 & 264 & 117 & 177 & 553 & 398 & 439 & 313 & 313 & 516 & 510 & 510 & 201 \\
\hline 120 & 253 & 311 & 248 & 162 & 493 & 436 & 264 & 264 & 552 & 281 & 281 & 230 \\
\hline 361 & 77 & 298 & 209 & 149 & 473 & 417 & 91 & 91 & 290 & 82 & 82 & 75 \\
\hline 576 & 160 & 234 & 107 & 171 & 429 & 448 & 295 & 295 & 409 & 30 & 30 & 351 \\
\hline 221 & 314 & 362 & 189 & 256 & 559 & 438 & 182 & 182 & 171 & 202 & 202 & 345 \\
\hline 91 & 69 & 74 & 202 & 255 & 490 & 407 & 544 & 544 & 26 & 309 & 309 & 229 \\
\hline 24 & 498 & 116 & 130 & \begin{tabular}{|l|l}
562 \\
\end{tabular} & 370 & 404 & 492 & 492 & 261 & 230 & 230 & 308 \\
\hline 67 & 110 & 231 & 227 & 190 & 270 & 405 & 243 & 243 & 567 & 297 & 297 & 65 \\
\hline 429 & 501 & 577 & 210 & 150 & 88 & 406 & 511 & 511 & 124 & 80 & 80 & 218 \\
\hline 537 & 278 & 6 & 212 & 145 & 6 & 366 & 165 & 165 & 138 & 398 & 398 & 24 \\
\hline 299 & 355 & 124 & 213 & 143 & 350 & 443 & 476 & 476 & 178 & 258 & 258 & 220 \\
\hline 437 & 218 & 554 & 186 & 129 & 523 & 437 & 190 & 190 & 50 & 41 & 41 & 410 \\
\hline 159 & 238 & 486 & 184 & 508 & 239 & 447 & 296 & 296 & 370 & 399 & 399 & 339 \\
\hline 296 & 18 & 576 & 185 & 198 & 553 & 434 & 224 & 224 & 347 & 221 & 221 & 512 \\
\hline 230 & 60 & 9 & 104 & 156 & 426 & 435 & 536 & 536 & 568 & 352 & 352 & 543 \\
\hline 478 & 345 & 291 & 92 & 240 & 432 & 408 & 90 & 90 & 444 & 286 & 286 & 165 \\
\hline 204 & 346 & 10 & 510 & 188 & 266 & 403 & 10 & 10 & 141 & 432 & 432 & 562 \\
\hline 302 & 365 & 84 & 422 & 245 & 410 & 419 & 206 & 206 & 54 & 11 & 11 & 56 \\
\hline 233 & 123 & 34 & 136 & 208 & 265 & 418 & 464 & 464 & 24 & 56 & 56 & 57 \\
\hline 286 & 135 & 3 & 126 & 135 & 354 & 445 & 37 & 37 & 185 & 534 & 534 & 178 \\
\hline 451 & 180 & 54 & 170 & 246 & 322 & 446 & 540 & 540 & 267 & 203 & 203 & 250 \\
\hline 165 & 444 & 353 & 256 & 125 & 315 & 573 & 558 & 558 & 223 & 57 & 57 & 12 \\
\hline
\end{tabular}




\begin{tabular}{|c|c|c|c|c|c|c|c|c|c|c|c|c|}
\hline 109 & 122 & 241 & 255 & 247 & 372 & 572 & 505 & 505 & 353 & 186 & 186 & 11 \\
\hline 252 & 120 & 571 & 230 & 148 & 176 & 444 & 212 & 212 & 200 & 433 & 433 & 53 \\
\hline 150 & 221 & 2 & 123 & 108 & 330 & 575 & 213 & 213 & 291 & 574 & 574 & 51 \\
\hline 41 & 24 & 222 & 190 & 510 & 314 & 574 & 531 & 531 & 414 & 308 & 308 & 104 \\
\hline 250 & 437 & 453 & 225 & 224 & 427 & 500 & 11 & 11 & 517 & 191 & 191 & 561 \\
\hline 21 & 159 & 212 & 68 & 281 & 207 & 433 & 123 & 123 & 520 & 133 & 133 & 263 \\
\hline 92 & 296 & 290 & 159 & 155 & 371 & 402 & 351 & 351 & 74 & 122 & 122 & 186 \\
\hline 316 & 230 & 536 & 258 & 283 & 435 & 432 & 513 & 513 & 546 & 67 & 67 & 566 \\
\hline 349 & 204 & 514 & 163 & 259 & 142 & 450 & 546 & 546 & 460 & 231 & 231 & 568 \\
\hline 541 & 165 & 312 & 275 & 230 & 3 & 457 & 506 & 506 & 97 & 2 & 2 & 270 \\
\hline 560 & 252 & 5 & 274 & 244 & 415 & 455 & 244 & 244 & 255 & 310 & 310 & 268 \\
\hline 547 & 41 & 174 & 218 & 220 & 526 & 451 & 205 & 205 & 542 & 148 & 148 & 169 \\
\hline 11 & 250 & 232 & 881 & 552 & 329 & 474 & 489 & 489 & 392 & 3 & 3 & 146 \\
\hline 43 & 21 & 205 & 273 & 238 & 1 & 452 & 194 & 194 & 554 & 428 & 428 & 89 \\
\hline 303 & 316 & 79 & 172 & 221 & 15 & 462 & 541 & 541 & 234 & 88 & 88 & 182 \\
\hline 161 & 547 & 91 & 128 & 199 & 501 & 461 & 271 & 271 & 449 & 188 & 188 & 362 \\
\hline 400 & 11 & 158 & 229 & 124 & 355 & 458 & 494 & 494 & 302 & 75 & 75 & 238 \\
\hline 475 & 303 & 421 & 280 & 227 & 120 & 479 & 210 & 210 & 12 & 289 & 289 & 296 \\
\hline 104 & 104 & 518 & 284 & 163 & 119 & 453 & 462 & 462 & 213 & 89 & 89 & 249 \\
\hline 528 & 543 & 14 & 149 & 258 & 333 & 481 & 530 & 530 & 532 & 161 & 161 & 557 \\
\hline 543 & 87 & 118 & 134 & 881 & 117 & 476 & 545 & 545 & 112 & 79 & 79 & 83 \\
\hline 59 & 119 & 300 & 176 & 338 & 577 & 478 & 512 & 512 & 137 & 337 & 337 & 281 \\
\hline 438 & 338 & 226 & 129 & 343 & 118 & 454 & 41 & 41 & 246 & 53 & 53 & 183 \\
\hline 553 & 477 & 516 & 220 & 159 & 469 & 456 & 529 & 529 & 336 & 712 & 712 & 108 \\
\hline 87 & 549 & 223 & 150 & 556 & 319 & 460 & 477 & 477 & 52 & 184 & 184 & 287 \\
\hline 119 & 566 & 213 & 198 & 104 & 334 & 477 & 493 & 493 & 34 & 272 & 272 & 280 \\
\hline 64 & 224 & 69 & 238 & 90 & 405 & 475 & 473 & 473 & 179 & 483 & 483 & 283 \\
\hline 338 & 272 & 324 & 337 & 243 & 406 & 459 & 559 & 559 & 475 & 276 & 276 & 135 \\
\hline 477 & 220 & 200 & 199 & 229 & 465 & 485 & 490 & 490 & 263 & 552 & 552 & 315 \\
\hline 549 & 399 & 517 & 556 & 275 & 466 & 482 & 270 & 270 & 502 & 290 & 290 & 546 \\
\hline 449 & 151 & 175 & 106 & 274 & 502 & 520 & 88 & 88 & 480 & 409 & 409 & 346 \\
\hline 234 & 284 & 414 & 561 & 218 & 379 & 491 & 350 & 350 & 13 & 26 & 26 & 278 \\
\hline 566 & 136 & 409 & 148 & 69 & 375 & 484 & 523 & 523 & 266 & 567 & 567 & 314 \\
\hline 440 & 65 & 276 & 105 & 231 & 571 & 521 & 239 & 239 & 175 & 178 & 178 & 237 \\
\hline 224 & 121 & 304 & 247 & 179 & 324 & 503 & 553 & 553 & 174 & 50 & 50 & 81 \\
\hline 272 & 106 & 567 & 206 & 178 & 321 & 505 & 266 & 266 & 393 & 347 & 347 & 69 \\
\hline 149 & 270 & 196 & 221 & 273 & 318 & 506 & 265 & 265 & 274 & 444 & 444 & 260 \\
\hline 220 & 362 & 162 & 224 & 226 & 310 & 490 & 322 & 322 & 117 & 24 & 24 & 289 \\
\hline 399 & 435 & 224 & 242 & 89 & 304 & 507 & 315 & 315 & 536 & 185 & 185 & 272 \\
\hline 446 & 169 & 310 & 171 & 187 & 147 & 504 & 176 & 176 & 15 & 267 & 267 & 513 \\
\hline 151 & 63 & 242 & 89 & 561 & 401 & 488 & 330 & 330 & 183 & 291 & 291 & 881 \\
\hline 504 & 315 & 26 & 226 & 151 & 525 & 492 & 314 & 314 & 514 & 74 & 74 & 26 \\
\hline 469 & 392 & 462 & 205 & 107 & 522 & 487 & 207 & 207 & 324 & 546 & 546 & 80 \\
\hline 284 & 26 & 78 & 154 & 180 & 484 & 499 & 142 & 142 & 196 & 460 & 460 & 342 \\
\hline 531 & 283 & 113 & 204 & 712 & 521 & 495 & 3 & 3 & 91 & 255 & 255 & 558 \\
\hline 136 & 271 & 344 & 712 & 239 & 475 & 489 & 526 & 526 & 577 & 302 & 302 & 204 \\
\hline 154 & 403 & 53 & 125 & 257 & 572 & 511 & 329 & 329 & 156 & 12 & 12 & 295 \\
\hline 9 & 298 & 11 & 259 & 241 & 453 & 509 & 1 & 1 & 344 & 336 & 336 & 21 \\
\hline 65 & 339 & 50 & 240 & 203 & 392 & 486 & 465 & 465 & 158 & 34 & 34 & 206 \\
\hline 293 & 281 & 119 & 237 & 237 & 437 & 497 & 466 & 466 & 51 & 179 & 179 & 60 \\
\hline 489 & 116 & 184 & 124 & 186 & 332 & 496 & 502 & 502 & 304 & 475 & 475 & 712 \\
\hline 567 & 202 & 179 & 231 & 280 & 331 & 498 & 379 & 379 & 486 & 263 & 263 & 271 \\
\hline 370 & 167 & 408 & 241 & 105 & 419 & 493 & 324 & 324 & 402 & 13 & 13 & 203 \\
\hline 121 & 313 & 186 & 246 & 204 & 418 & 483 & 321 & 321 & 224 & 266 & 266 & 544 \\
\hline 106 & 53 & 191 & 178 & 136 & 302 & 494 & 318 & 318 & 241 & 393 & 393 & 565 \\
\hline 388 & 329 & 318 & 90 & 106 & 381 & 508 & 310 & 310 & 453 & 117 & 117 & 105 \\
\hline 570 & 190 & 52 & 272 & 181 & 359 & 510 & 304 & 304 & 47 & 183 & 183 & 264 \\
\hline 391 & 184 & 336 & 160 & 153 & 454 & 517 & 147 & 147 & 346 & 514 & 514 & 205 \\
\hline 166 & 310 & 185 & 191 & 152 & 483 & 515 & 525 & 525 & 118 & 196 & 196 & 288 \\
\hline 495 & 552 & 47 & 192 & 197 & 393 & 516 & 522 & 522 & 293 & 577 & 577 & 267 \\
\hline 312 & 185 & 346 & 151 & 284 & 363 & 514 & 475 & 475 & 408 & 344 & 344 & 107 \\
\hline 270 & 191 & 239 & 180 & 560 & 402 & 512 & 332 & 332 & 315 & 158 & 158 & 545 \\
\hline 362 & 236 & 315 & 245 & 269 & 307 & 513 & 331 & 331 & 236 & 51 & 51 & 207 \\
\hline 371 & 153 & 547 & 239 & 182 & 33 & 519 & 302 & 302 & 307 & 304 & 304 & 33 \\
\hline 435 & 419 & 419 & 279 & 183 & 459 & 532 & 307 & 307 & 569 & 486 & 486 & 106 \\
\hline 540 & 276 & 293 & 285 & 272 & 467 & 524 & 33 & 33 & 462 & 402 & 402 & 265 \\
\hline 5 & 147 & 51 & 244 & 557 & 99 & 527 & 467 & 467 & 571 & 224 & 224 & 266 \\
\hline 169 & 334 & 343 & 203 & 184 & 141 & 526 & 99 & 99 & 518 & 241 & 241 & 284 \\
\hline 63 & 467 & 15 & 153 & 185 & 130 & 528 & 141 & 141 & 343 & 47 & 47 & 475 \\
\hline 315 & 483 & 112 & 152 & 262 & 344 & 525 & 130 & 130 & 1 & 346 & 346 & 273 \\
\hline 392 & 48 & 301 & 557 & 337 & 48 & 480 & 344 & 344 & 422 & 118 & 118 & 160 \\
\hline 466 & 337 & 147 & 560 & 206 & 422 & 523 & 48 & 48 & 232 & 408 & 408 & 217 \\
\hline 384 & 459 & 459 & 565 & 160 & 145 & 518 & 145 & 145 & 204 & 315 & 315 & 338 \\
\hline 317 & 192 & 33 & 207 & 565 & 143 & 522 & 143 & 143 & 119 & 236 & 236 & 136 \\
\hline 26 & 418 & 154 & 243 & 261 & 129 & 531 & 129 & 129 & 239 & 307 & 307 & 277 \\
\hline 283 & 359 & 307 & 257 & 279 & 508 & 530 & 508 & 508 & 318 & 462 & 462 & 285 \\
\hline 271 & 335 & 305 & 183 & 563 & 198 & 529 & 198 & 198 & 147 & 343 & 343 & 241 \\
\hline 403 & 241 & 99 & 197 & 68 & 510 & 542 & 510 & 510 & 68 & 1 & 1 & 508 \\
\hline 298 & 68 & 379 & 269 & 191 & 552 & 547 & 552 & 552 & 242 & 232 & 232 & 48 \\
\hline
\end{tabular}




\begin{tabular}{|c|c|c|c|c|c|c|c|c|c|c|c|c|}
\hline 339 & 33 & 418 & 262 & 192 & 199 & 543 & 199 & 199 & 419 & 204 & 204 & 153 \\
\hline 281 & 307 & 321 & 261 & 205 & 227 & 546 & 227 & 227 & 379 & 239 & 239 & 152 \\
\hline 439 & 251 & 97 & 181 & 251 & 343 & 544 & 343 & 343 & 146 & 318 & 318 & 393 \\
\hline 381 & 319 & 1 & 182 & 271 & 275 & 545 & 275 & 275 & 321 & 147 & 147 & 344 \\
\hline 533 & 227 & 146 & 251 & 285 & 274 & 535 & 274 & 274 & 162 & 68 & 68 & 307 \\
\hline 45 & 577 & 365 & 271 & 558 & 179 & 555 & 179 & 179 & 99 & 419 & 419 & 179 \\
\hline 46 & 402 & 137 & 161 & 154 & 273 & 536 & 273 & 273 & 305 & 146 & 146 & 459 \\
\hline 465 & 343 & 422 & 278 & 569 & 226 & 550 & 226 & 226 & 418 & 162 & 162 & 227 \\
\hline 484 & 508 & 12 & 335 & 217 & 241 & 563 & 241 & 241 & 192 & 418 & 418 & 302 \\
\hline 116 & 510 & 13 & 286 & 270 & 153 & 553 & 153 & 153 & 335 & 192 & 192 & 510 \\
\hline 202 & 199 & 156 & 563 & 268 & 152 & 570 & 152 & 152 & 365 & 335 & 335 & 191 \\
\hline 176 & 152 & 335 & 558 & 207 & 184 & 554 & 184 & 184 & 33 & 365 & 365 & 192 \\
\hline 318 & 333 & 302 & 569 & 263 & 185 & 548 & 185 & 185 & 547 & 33 & 33 & 552 \\
\hline 502 & 363 & 273 & 567 & 278 & 337 & 551 & 337 & 337 & 227 & 547 & 547 & 359 \\
\hline 118 & 393 & 152 & 217 & 559 & 261 & 560 & 261 & 261 & 251 & 227 & 227 & 251 \\
\hline 167 & 179 & 501 & 270 & 564 & 68 & 564 & 68 & 68 & 501 & 251 & 251 & 467 \\
\hline 70 & 344 & 251 & 268 & 567 & 191 & 556 & 191 & 191 & 363 & 363 & 363 & 184 \\
\hline 114 & 304 & 68 & 263 & 161 & 192 & 569 & 192 & 192 & 154 & 154 & 154 & 185 \\
\hline 117 & 302 & 227 & 277 & 335 & 251 & 549 & 251 & 251 & 28 & 28 & 28 & 335 \\
\hline 313 & 286 & 467 & 559 & 286 & 154 & 559 & 154 & 154 & 152 & 152 & 152 & 363 \\
\hline 53 & 161 & 469 & 564 & 267 & 569 & 562 & 569 & 569 & 48 & 48 & 48 & 68 \\
\hline 73 & 475 & 359 & 236 & 264 & 567 & 561 & 567 & 567 & 459 & 459 & 459 & 343 \\
\hline 248 & 469 & 192 & 267 & 236 & 161 & 566 & 161 & 161 & 359 & 359 & 359 & 567 \\
\hline 329 & 154 & 319 & 264 & 277 & 335 & 568 & 335 & 335 & 273 & 273 & 273 & 161 \\
\hline 10 & 567 & 28 & 276 & 566 & 286 & 557 & 286 & 286 & 199 & 199 & 199 & 236 \\
\hline 190 & 318 & 334 & 566 & 568 & 236 & 558 & 236 & 236 & 469 & 469 & 469 & 337 \\
\hline 273 & 118 & 363 & 568 & 266 & 566 & 565 & 566 & 566 & 319 & 319 & 319 & 276 \\
\hline 461 & 117 & 199 & 266 & 265 & 568 & 552 & 568 & 568 & 334 & 334 & 334 & 154 \\
\hline 354 & 273 & 48 & 265 & 276 & 276 & 567 & 276 & 276 & 467 & 467 & 467 & 286 \\
\hline 231 & 38 & 38 & 38 & 38 & 38 & 38 & 38 & 38 & 38 & 38 & 38 & 38 \\
\hline 0 & 0 & 0 & 0 & 0 & 0 & 0 & 0 & 0 & 0 & 0 & 0 & 0 \\
\hline
\end{tabular}

Table 14 Statistics of 120 important node parameters

\begin{tabular}{|c|c|c|c|c|c|c|c|c|c|c|c|}
\hline \multicolumn{3}{|c|}{$\begin{array}{l}\text { degree, authority } \\
\text { hub、 eigenvector } \\
\text { centrality, triangles }\end{array}$} & \multicolumn{5}{|c|}{$\begin{array}{l}\text { Closeness Centrality、Harmonic } \\
\text { closness centrality、Betweeness } \\
\text { centrality }\end{array}$} & \multicolumn{2}{|c|}{$\begin{array}{l}\text { clustering } \\
\text { coefficient }\end{array}$} & \multicolumn{2}{|c|}{ pagerank } \\
\hline 17 & 448 & 386 & 519 & 14 & 323 & 52 & 82 & 525 & 375 & 36 & 464 \\
\hline 394 & 369 & 378 & 468 & 23 & 7 & 299 & 534 & 381 & 466 & 219 & 189 \\
\hline 413 & 368 & 383 & 470 & 532 & 325 & 312 & 80 & 275 & 465 & 155 & 482 \\
\hline 417 & 441 & 447 & 364 & 62 & 320 & 354 & 79 & 145 & 401 & 95 & 25 \\
\hline 396 & 404 & 382 & 22 & 15 & 576 & 291 & 348 & 454 & 521 & 209 & 254 \\
\hline 436 & 438 & 380 & 98 & 5 & 233 & 81 & 501 & 522 & 484 & 64 & 127 \\
\hline 442 & 440 & 571 & 16 & 306 & 317 & 260 & 365 & 143 & 572 & 544 & 527 \\
\hline 439 & 406 & 443 & 389 & 6 & 539 & 308 & 252 & 129 & 332 & 135 & 225 \\
\hline 367 & 405 & 366 & 431 & 353 & 300 & 28 & 298 & 355 & 331 & 159 & 457 \\
\hline 407 & 384 & 373 & 395 & 471 & 297 & 538 & 437 & 120 & 130 & 566 & 495 \\
\hline
\end{tabular}




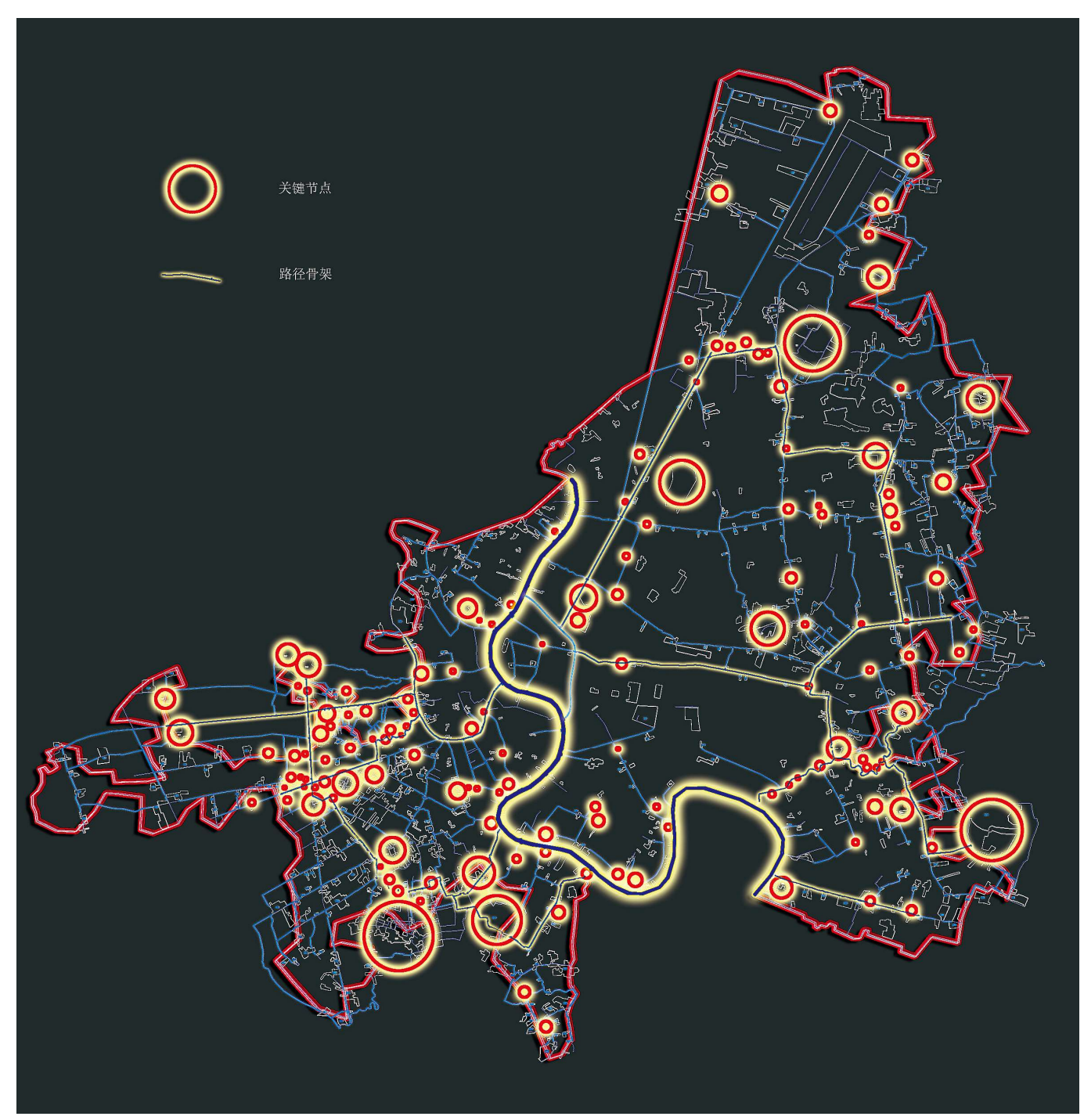

Fig.20 Flood safety pattern in the built-up area of Bangkok

\section{Discussion}

4.1 Dynamic security pattern on the horizontal

The urban flood safety pattern in Bangkok is a changing dynamic pattern, and this dynamic is reflected in both temporal and spatial dimensions. Temporal dynamics means that the safety pattern can be adjusted and optimized at any time in response to climate change, urban development and changes in government decisions, and is a dynamic safety pattern that can be chosen and defended. Spatially dynamic refers to the topological evolution of the geographic landscape layout of the security pattern guided by complex network theory. Urban development and flooding pattern is a process of mutual influence and mutual choice, both of them play each other in time and space, constantly adjust and promote each other, and finally form a dynamic balance of security, stability and development. At the same time, the urban flood safety pattern is an open system in a broad sense, allowing various factors 
that affect and interfere with the structure and function of the water network to participate in the superposition, such as water transport traffic, water markets, water body landscape, water ecological communities, etc., and through the horizontal game integration with various factors, finally forming an urban water network safety pattern based on urban flood safety.

4.2 Hierarchical security pattern in the vertical

The flood safety pattern in the built-up area of Bangkok is vertically divided into three levels: macro, meso and micro. The analysis and study of the current situation of the water system and the storage network model in the built-up area of Bangkok using complex network theory identifies the key nodes and important paths, and constructs the flood safety pattern at the macro level by combining the current conditions and the characteristics of urban development. Through the network model analysis construction, the Bangkok water system storage network model has an obvious association structure, and the area covered by the association is analyzed twice by using the self-similarity feature to establish a mesoscopic scale regional-association level safety pattern. Combining with the characteristics of urban neighborhoods and geographic landscape environment, the micro-scale landscape design of the storage point-water system is carried out, so that it not only has the practical function of urban flood storage, but also can be integrated with the urban landscape and landscape, creating a landscape leisure pattern for citizens' leisure activities and gathering and communication.

\subsection{Problems}

The theoretical analysis and research related to complex networks mainly focus on virtual networks, although there are also some physical space networks that have been studied using some of the attributes of complex networks, but in general, a more mature research method has not been formed. The active exploration in this paper has analyzed and processed a large amount of data on the basis of no case to follow, and although certain theoretical methods have been formed and some results have been achieved, there are inevitably imperfections and lack of depth, and further in-depth research is needed.

The implementation of the land privatization policy in Bangkok and the construction of a flood safety pattern in the built-up area of Bangkok will require the Bangkok Metropolitan Administration to reserve and purchase land at key nodes in the safety pattern in advance and to plan and control them. At the same time, the construction of the safety pattern is not only a technical and engineering solution, but also involves political, economic, social and cultural aspects of the city, which will be a long and complicated process given the current construction and development of Bangkok city.

\section{Conclusions}

The implementation of the land privatization policy in Bangkok and the construction of a flood safety pattern in the built-up area of Bangkok will require the Bangkok Metropolitan Administration to reserve and purchase land at key nodes in the safety pattern in advance and to plan and control them. At the same time, the construction of the safety pattern is not only a technical and engineering solution, but also involves political, economic, social and 
cultural aspects of the city, which will be a long and complicated process given the current construction and development of Bangkok city.

Author Contributions: Conceptualization, Zhou Yingying and Li Shuaibo; methodology, Zhou Yingying; software, Li Shuaibo; validation, Li Shuaibo and Zhou Yingying ; formal analysis, Li Shuaibo; investigation, Zhou Yingying; resources, Zhou Yingying; data curation,Li Shuaibo and Zhou Yingying; writing - original draft preparation, Zhou Yingying; writing-review and editing, Li Shuaibo; visualization, Li Shuaibo; supervision, Zhou Yingying. All authors have read and agreed to the published version of the manuscript.

Funding: This research received no external funding.

Data Availability Statement: Not applicable.

Acknowledgements:Not applicable.

Conflicts of Interest: The authors declare no conflict of interest.

Availability of data and materials: The datasets used and/or analysed during the current study are available from the corresponding author on reasonable request.

\section{References}

Albert R, B. A. (2002). Statistical mechanics of complex networks. Review of Modern Physics, pp. 74(1):47-97.

Ben-Naim, E. (2004). Complex Networks. Springer .

exclusive secrets, bangkok's next 7 potential investment golden places! $(2019,124)$. Retrieved from sohu: https://www.sohu.com/a/291113405_117348

HAN Ruidan, Z. L. (2017). Urban expansion and its ecological environmental effects in Bangkok, Thailand. Acta Ecologica Sinica , pp. 37(19): 6322-6334.

J.M ENewman. (2003). The structure and function of complex networks. SIAM Review, pp 45;167-256.

J.M ENewman. (2003). The structure and function of complex networks. SIAM Review, pp 45;167-256.

Newman. (2003). The structure and function of complex networks. SIAM Review , pp. 45;167-256.

Newman, B. W. (2006). The Structure and Dynamics of Networks. Princeton University Press.

Noble, N. E. $(2009,159$ (10)). The clustering coefficient of a scale-free random graph . Discrete Applied Mathematics, pp. 953-965.

S.Boccaletti. (2006). Complex Networks: Structure and dynamics. Physics Reports , pp. 424 (2006)

175-308.

Watts D J, S. S. (1998). Collective dynamics of 'small-world' networks. Nature, p. 393(6684):440. 